Check for updates

Cite this: RSC Adv., 2019, 9, 37009

\title{
Recent advances in metallopolymer-based drug delivery systems
}

\author{
Gulzhian I. Dzhardimalieva, ${ }^{\text {ab }}$ Lev N. Rabinskiy, $^{\text {b Kamila A. Kydralieva }}{ }^{\text {b }}$ \\ and Igor E. Uflyand (D) ${ }^{\mathrm{C}}$
}

Metallopolymers (MPs) or metal-containing polymers have shown great potential as new drug delivery systems (DDSs) due to their unique properties, including universal architectures, composition, properties and surface chemistry. Over the past few decades, the exponential growth of many new classes of MPs that deal with these issues has been demonstrated. This review presents and assesses the recent advances and challenges associated with using MPs as DDSs. Among the most widely used MPs for these purposes, metal complexes based on synthetic and natural polymers, coordination polymers, metal-organic frameworks, and metallodendrimers are distinguished. Particular attention is paid to the stimulus- and multistimuli-responsive metallopolymer-based DDSs. Of considerable interest is the use of MPs for combination therapy and multimodal systems. Finally, the problems and future prospects of using metallopolymer-based DDSs are outlined. The bibliography includes articles published over the past five years.

Received 24th August 2019

Accepted 6th November 2019

DOI: $10.1039 / \mathrm{c} 9 \mathrm{ra06678k}$

rsc.li/rsc-advances

'Department of Chemistry, Southern Federal University, B. Sadovaya Str. 105/42, Rostov-on-Don, 344006 Russian Federation. E-mail: ieuflyand@sfedu.ru
${ }^{a}$ Laboratory of Metallopolymers, The Institute of Problems of Chemical Physics RAS, Academician Semenov Avenue 1, Chernogolovka, Moscow Region, 142432 Russian Federation. E-mail: dzhardim@icp.ac.ru

${ }^{b}$ Moscow Aviation Institute (National Research University), Volokolamskoe Shosse, 4, Moscow 125993, Russia.E-mail:f9_dec@mai.ru; kamila.kydralieva@gmail.com

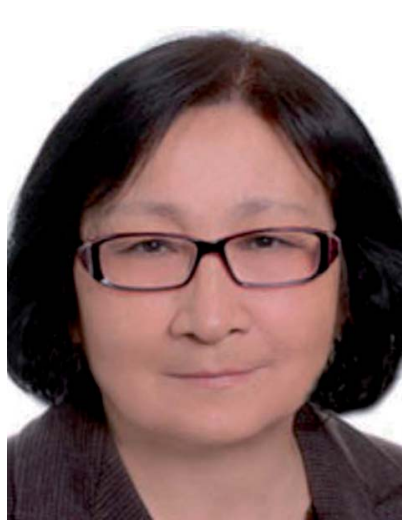

Gulzhian I. Dzhardimalieva, Dr Sci. (Chem.), PhD is the Head of Laboratory, Institute of Problems of Chemical Physics Russian Academy of Sciences and Professor of Department of Applied Mechanics, Moscow Aviation Institute (National Research University). She received her PhD in 1987 from the Institute of Chemical Physics, RAS in Moscow, and her Doctorate in Chemistry in 2010 from Institute of Problems of Chemical Physics RAS in Chernogolovka. The scope of her scientific interest is: metal-containing monomers and polymers on their base, macromolecular metal carboxylates, coordination polymers, metallopolymer nanocomposites, and nanomaterials. She has been as invited researcher of Auburn University, USA (2001), Warsaw Technology University, Poland (2002, 2011), Bremen University, Germany (2007), Turin University, Italy (2008), and Institute of composite and biomedical materials CNR, Neapol, Italy (2011, 2013). She was issue editor of Journal of Inorganic and Organometallic Polymers and Materials (2016, Vol. 26, 6).

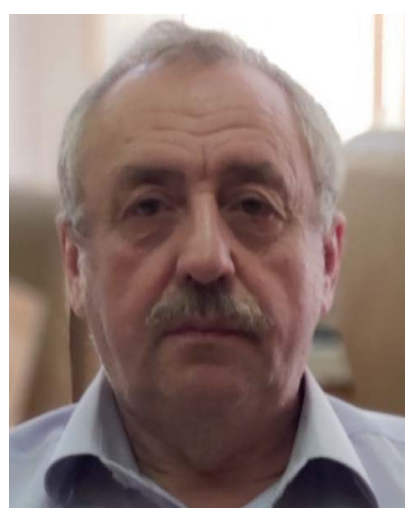

Lev Rabinskiy, Dr Sci. (Phys. Math). Director of Institute for Engineer Education and Head of Department of Constructional Materials, Materials in Moscow Aviation Institute (National Research University). Areas of expertise include development of applied and basic research in the area of composite materials for aerospace; investigation of high temperature coatings for oxidation and erosion protection of heat-resistant carbonaceous materials in high speed flows; optimization of process technology for the production of polymer composites; modelling mechanical, thermal analysis and viscoelasticity of polymer and polymer fibre reinforced systems, estimation of structural stresses in composites with whiskerized fibers. 


\section{Introduction}

The administration of drugs is one of the main methods of pharmaceutical and medicinal use. ${ }^{1}$ However, traditional direct drug administration to patients is limited due to poor solubility, undesirable side effects, limited cancer targeting, poor pharmacokinetics and biodistribution, and rapid metabolization and excretion. ${ }^{2,3}$ Despite the wide arsenal of drugs used in medicine, one of the most important problems remains their targeted delivery in order to increase the effectiveness of treatment. Drug delivery is the most promising approach to solving the problem of the safe transportation of a drug through the body to achieve its target., ${ }^{4,5}$ Targeted drug delivery will solve several problems: protect drugs from degradation and unwanted interactions with biological molecules, increase the selective absorption of drugs by tumor cells, monitor pharmacokinetics, and increase the bioavailability of drugs inside tumor cells. Targeted delivery combined with controlled drug release plays a key role in the future of personalized medicine. Key features of the drug delivery system (DDSs) include the following three indicators: effective drug encapsulation, successful delivery of these drugs to the target area of the body, and successful drug release there. Drugs equipped with a delivery system have several advantages compared to free drugs: they increase the solubility of hydrophobic drugs, improve their penetration into cells, improve pharmacokinetics, and many drugs have the ability to overcome membrane and blood-brain barriers.

DDS includes two main components: a carrier and a drug, and its optimal properties are controlled by careful selection of these components. ${ }^{6}$ To date, numerous DDSs have been created that can reduce side effects and increase therapeutic efficacy. ${ }^{7-9}$ Thus, a variety of inorganic materials were used, including carbon (nanotube, nanodiamond, graphene), ${ }^{\mathbf{1 0 , 1 1}}$ magnetic nanoparticles (NPs) of iron oxide, ${ }^{12,13}$ mesoporous silica nanoparticles (MSNPs), ${ }^{\mathbf{1 4}, \mathbf{1 5}}$ gold (gold nanorod, nanocage, and $\mathrm{NP}){ }^{16,17}$ and rare earth upconversion nanoparticles. ${ }^{18,19}$ Among
DDSs based on organic materials, we note liposomes ${ }^{\mathbf{2 0 , 2 1}}$ and lipid particles. ${ }^{22,23}$ Although some of these DDSs showed promising results and a number of formulations were approved for use in the clinic, the problem remains unresolved. In addition, all DDSs have their own limitations in bio-application, for example, liposomes usually suffer from low loads, and inorganic porous materials have undesirable toxicity and high degradability.

In recent years, researchers' attention to polymers-based DDSs has grown significantly due to their flexibility, the diversity of polymers in terms of composition and properties, their easy functionalization, and the possibility of smart behavior in response to environmental stimuli. ${ }^{24,25}$ Due to the difference of several orders of magnitude between the size of polymeric materials and the size of, for example, cancer cells, polymeric materials are ideally suited as in vivo DDSs. ${ }^{26-33}$ Three different methods for producing polymer-based DDSs have been developed: (1) physical encapsulation, (2) complexation of drugs with donor atoms of the polymer chains, and (3) conjugation of drugs with a polymeric carrier via a linker (polymeric prodrug). The undoubted advantage of the latter method is the delay in the release and even the trigger of the release when the DDS reaches its target.

Metallopolymers (MPs) or metal-containing polymers are one of the main classes of polymers discovered in the 20th century. ${ }^{34-41}$ Various locations of metal centers from main group metals to transition metals and lanthanides relative to the polymer backbone were described by Wolf as Type I, Type II or Type III (Fig. 1), in which the metal center is either tethered to the polymer backbone by an organic linker, either covalently coupled to the backbone, or directly included in the polymer backbone, respectively. ${ }^{42,43}$ As a further subdivision, MPs are linear, highly branched, or dendritic. The bonds of metals with the polymer are covalent, which leads to essentially irreversible or 'static' binding, or noncovalent, which could allow potentially reversible 'dynamic' binding.

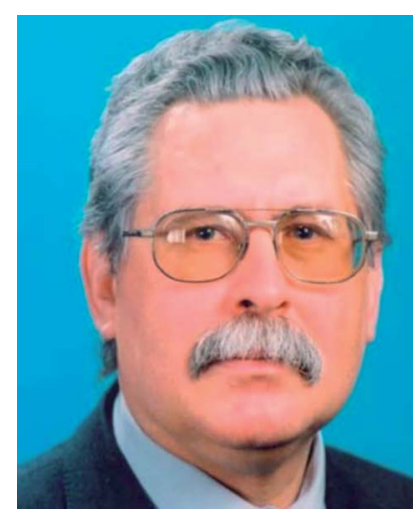

Prof. Igor E. Uflyand, Dr Sci. (Chem.) is the Head of Chemistry Department, Southern Federal University, Rostov-onDon, Russia. He was born in 1956 and graduated from Rostov State University, Department of Physical and Colloid Chemistry. Prof. Uflyand received his PhD in 1981 and his Doctorate in Chemistry in 1996 from the Rostov State University. $\mathrm{He}$ received the title of Professor in 1996. The scope of his scientific interest is: coordination and organometallic chemistry, metal chelate monomers and polymers on their basis, metal-containing polymers, catalysis by metal complexes, nanomaterials. 


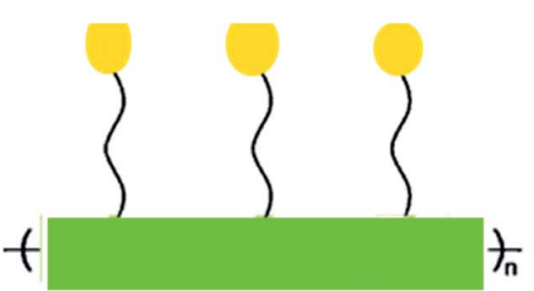

Type I

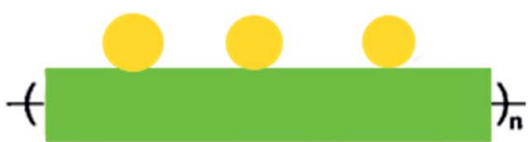

Type II

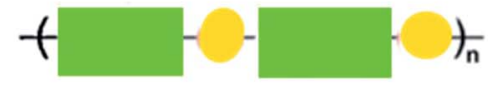

Type III = polymer backbone $=$ metal center

Fig. 1 Structural representations of Wolf Type I-III metallopolymers.

Characteristic features, allowing reliably identify MPs as particular class of chemical compounds, is the presence of the polymeric chain (organic, inorganic, hybrid or biological nature) and metal centers. Their properties can be adjusted by changing the interaction strength of metal centers with the polymer backbone. ${ }^{44-48}$ MPs are highly interesting materials with properties combining the technological advantages of polymers with the functionality of metal centers, therefore, these compounds have outstanding potential for use in a broad range of advanced sustainable applications including functional materials, ${ }^{49-55}$ catalysis and biocatalysis, ${ }^{56-63}$ electroactive, $^{64}$ photoactive, ${ }^{65-70}$ conducting ${ }^{71}$ and magnetic ${ }^{45}$ materials, as well as environment ${ }^{72}$ and nanomaterials science. $^{73-80}$
MPs have been known for a long time but have recently become the subject of large development. Over the years, the development of MP chemistry has been hampered by the lack of readily available synthesis techniques, difficulties in purifying the end products and convincing methods of their identification. In the early 1990s, a significant breakthrough was made in the development of new synthetic approaches (for example, polycondensation, ring-opening polymerization, electropolymerization, living ionic polymerization, controlled radical polymerization) and expanded access to characterization tools (for example, MALDI-TOF, ESI-MS, XPS, electron microscopy, spatially resolved optical spectroscopy), which allowed the production of metal-containing polymer materials with a welldefined spatial architectures..$^{81} \mathrm{~A}$ variety of protocols for the

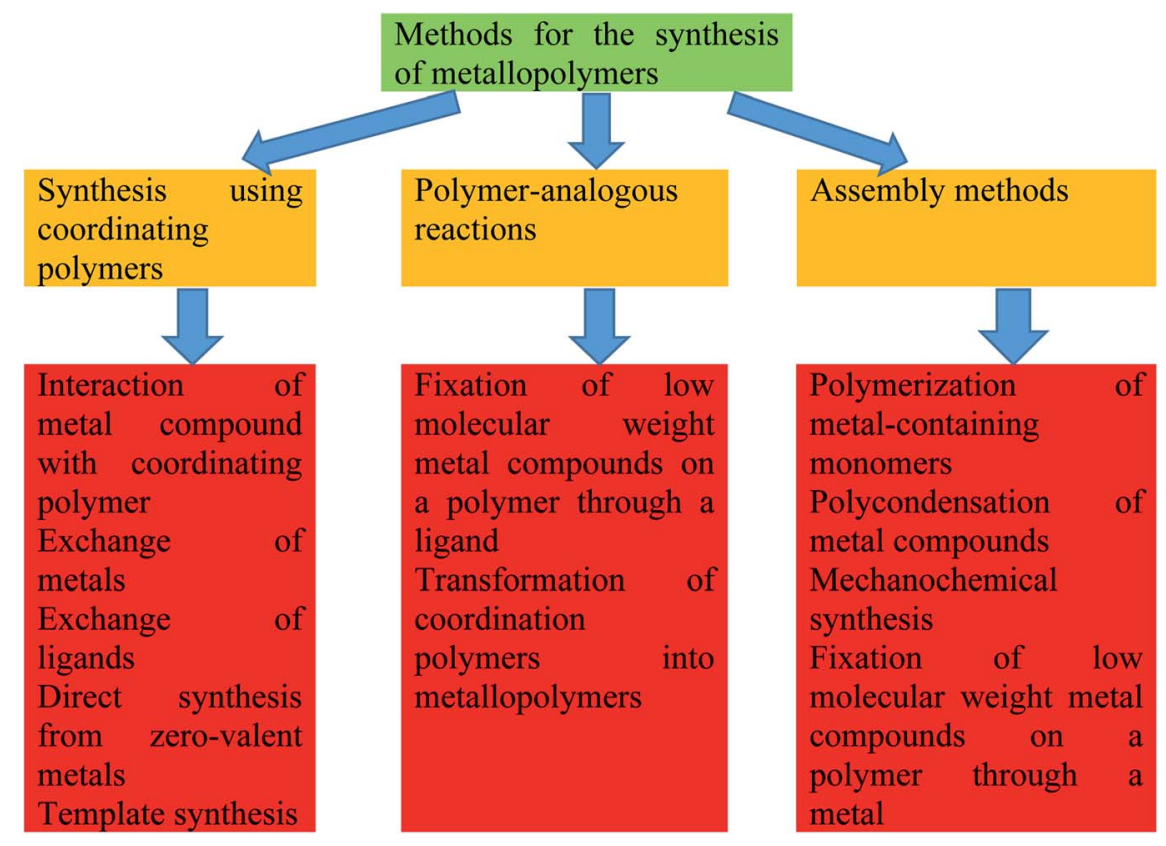

Fig. 2 Classification of the methods for the synthesis of metallopolymers of Type I and II. 


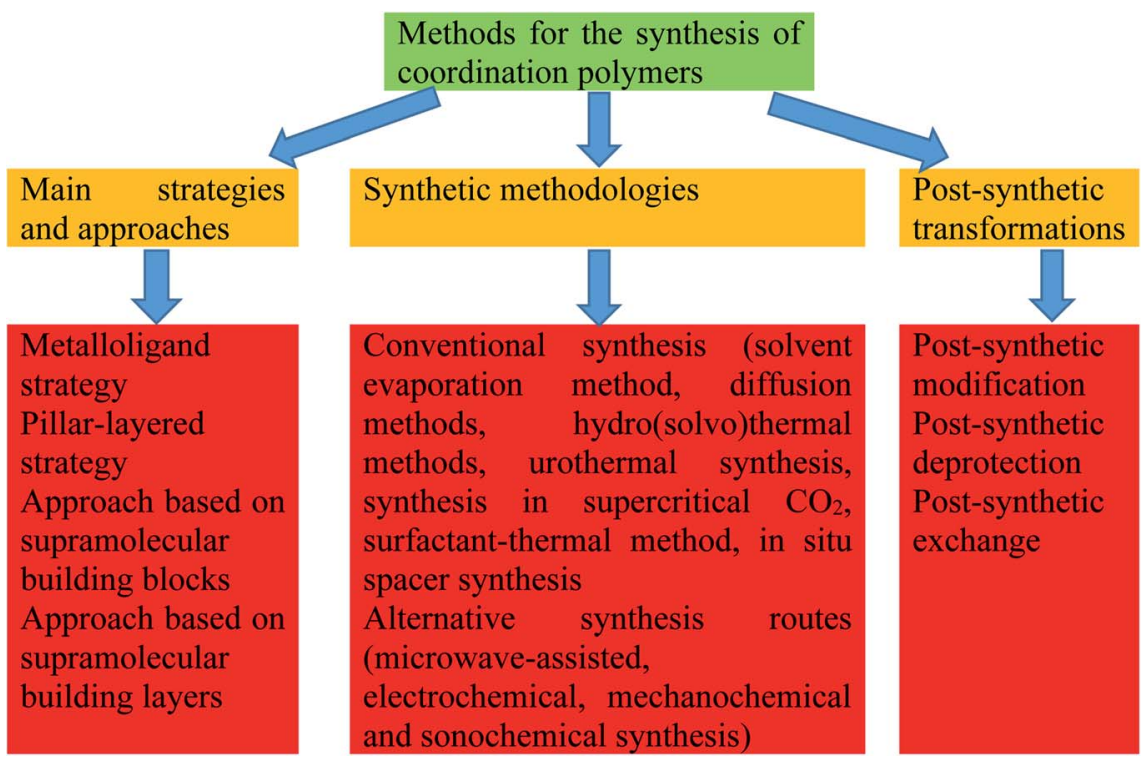

Fig. 3 The main strategies and approaches, synthetic methodologies and post-synthetic transformations of coordination polymers.

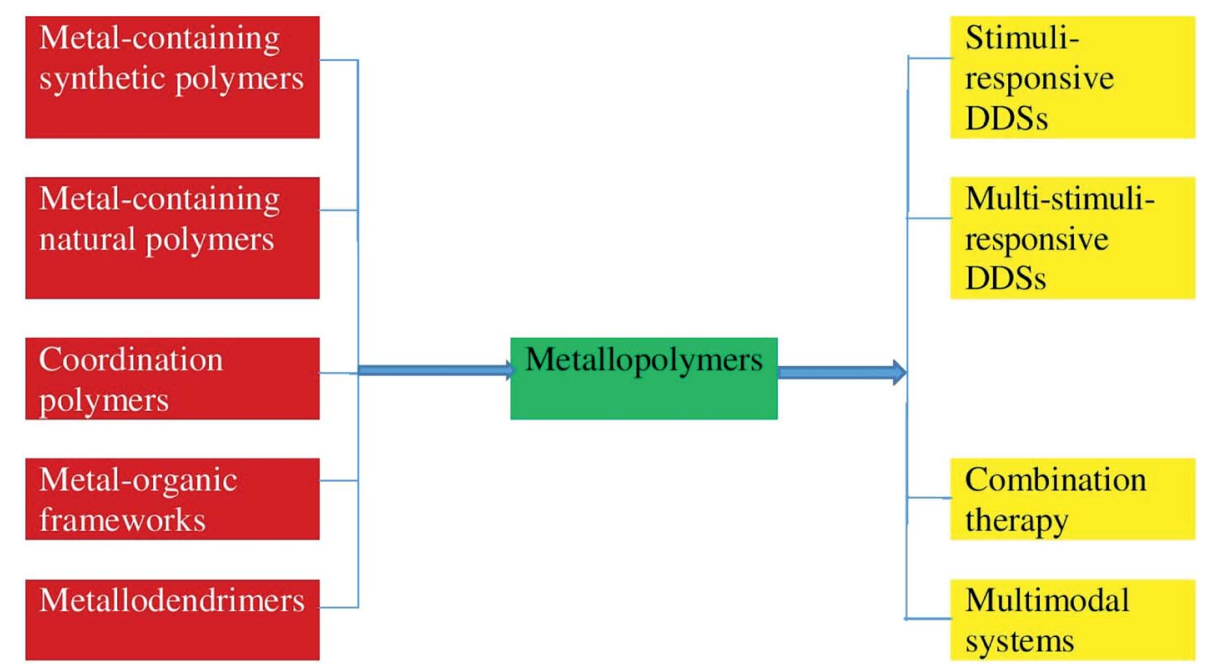

Fig. 4 The main types of metallopolymers-based DDSs, as well as use MPs as stimuli- and multistimuli-responsive DDSs, for combination therapy and as multimodal systems.
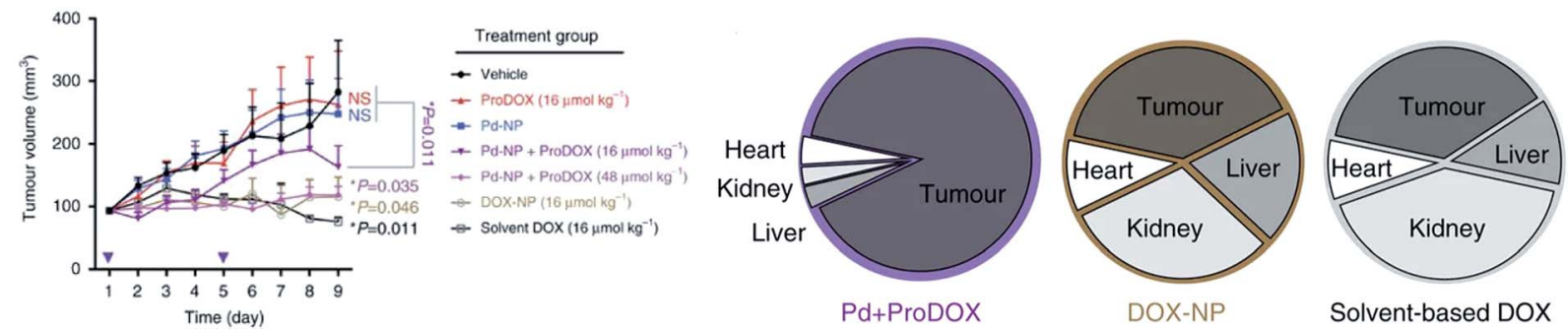

Fig. 5 (left) HT1080 tumor growth profile during treatment; (right) pie chart showing the distribution of nuclear DOX accumulation in orthotopic ovarian tumors and key organs associated with toxicity [reprinted from ref. 141 under a Creative Commons Attribution 4.0 International License]. 


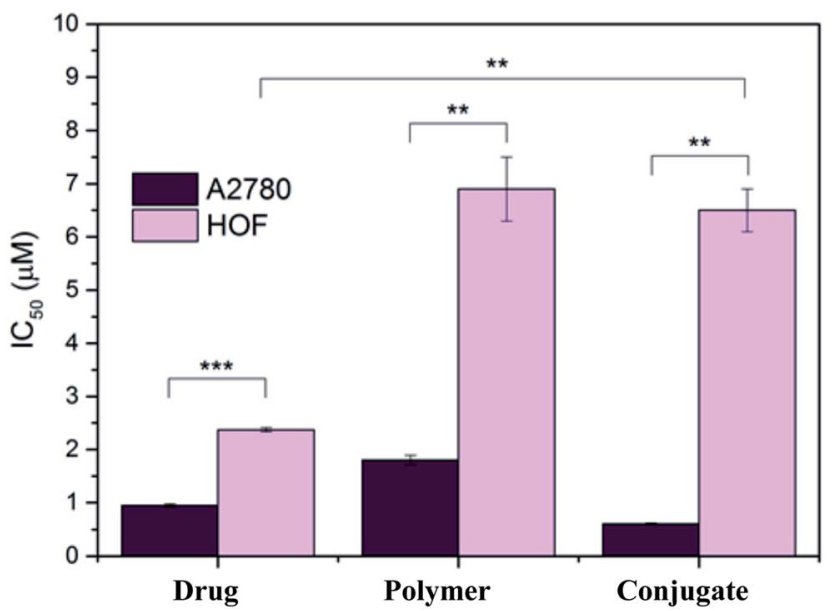

Fig. 6 Antiproliferative activity of free organoiridium complex, drugbearing polymer and drug-bearing conjugate in A2780 (cancer, dark) and HOF (healthy, light) ovarian cells. ${ }^{* *} p<0.01,{ }^{* * *} p<0.001$ [reprinted with permission from ref. 142. Copyright 2018 American Chemical Society].

synthesis of MPs of Type I and II were established by developing new synthetic approaches that are compatible with the presence of metal centers (Fig. 2). ${ }^{34,82-94}$

Coordination polymers (CPs) relate to MPs of Type III containing metal in the main chain, whose special feature is breaking of a polymer chain after a metal is removed. Because they contain large voids in the crystal structure, they were called porous coordination polymers or metal-organic frameworks (MOFs). Over the last decade, MOFs have received a great deal of interest in materials science due to their excellent material properties such as high surface area, porosity, high chemical and thermal stability, luminescence, high sorptive capacity, and potential use in a wide range of applications..$^{95-106}$ To date, around 20000 MOFs have been produced for a range of applications with different combinations of metal nodes and organic linkers. To date, a wide variety of strategies and approaches to the design of coordination polymers have been developed (Fig. 3). ${ }^{107-113}$

Already early pioneer work showed a high potential of MPs in biomedical applications. The construction of MPs is a new strategy for significantly increase the effectiveness of biomedical materials, in particular, to address current issues of traditional methods of treatment and prophylactics. ${ }^{4,114-132}$ Research on MPs in the biomedical field is impressive. Materials scientists are designing MPs as innovative solutions to improve the effectiveness of DDSs. Combined with the a rich and evergrowing array of ligands and synthesis methods for varieties of the metals, these compounds have outstanding potential for use in a wide variety of DDSs.

To the best of our knowledge, there is no general information on the use of metallopolymers as DDSs. The present review aims to describe the latest advances (over the last 5 years) in the

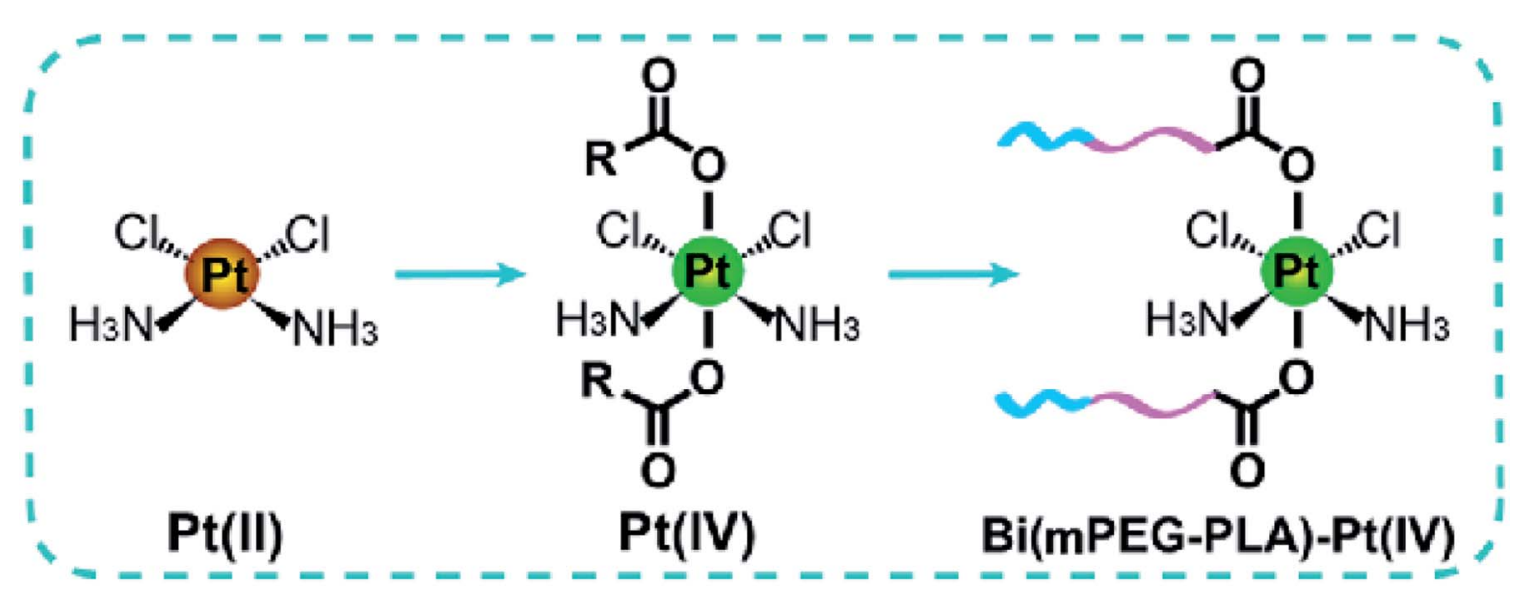

\section{Bi(mPEG-PLA)-Pt(IV)}

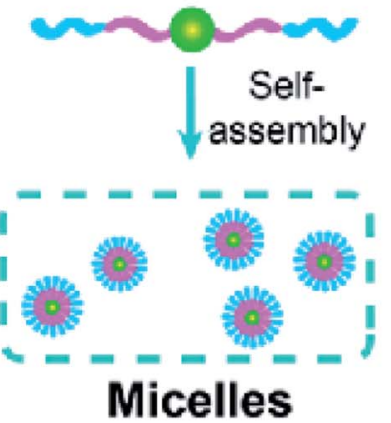

Fig. 7 Pt(Iv) drugs as crosslinking agents of NPs. Self-assembly of bis(mPEG-b-PLA)-Pt(Iv) conjugates in micelles [reprinted with permission from ref. 143. Copyright 2015, American Chemical Society]. 
A

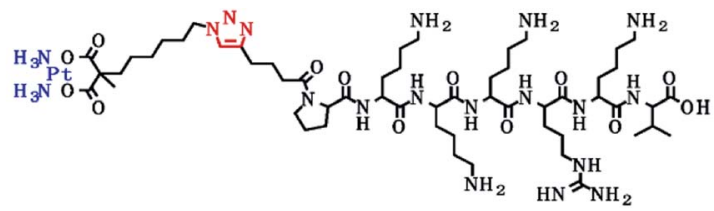

B
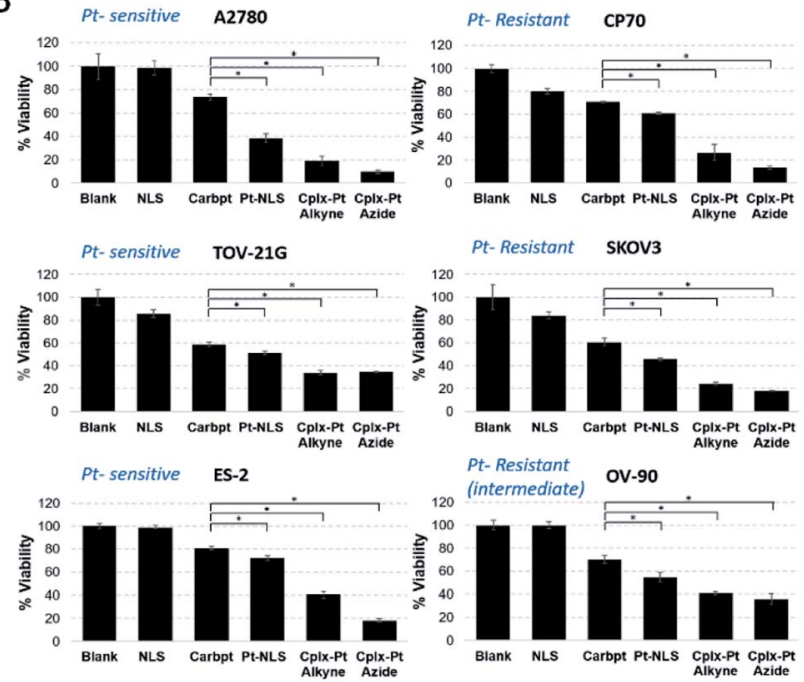

Fig. 8 (A) The chemical structure of the Pt(II)-NLS hybrid. (B) Viability of platinum-sensitive (left) and resistant (right) cells after $72 \mathrm{~h}$ incubation with Pt(II)-NLS hybrid and controls. *Each column represents the mean and standard deviation of $N=3$ and $p<0.005$ [reprinted with permission from ref. 147. Copyright 2018, American Chemical Society].

field of metallopolymers-based DDSs. The review will mainly discuss the main types of metallopolymers-based DDSs, as well as use MPs as stimuli- and multistimuli-responsive DDSs, for combination therapy and as multimodal systems (Fig. 4). We are not trying to give an exhaustive analysis of the entire array of experimental data to date. The main focus will be paid to the main directions of modern research in this interesting field.

\section{The main types of metallopolymers-based drug delivery systems}

The use of MPs as DDSs attracts considerable attention due to their unique tailorable structures and physicochemical properties, namely, given spatial organization, in many cases nanoscale size, abundant exterior or interior functionalities and universal structural control. In this section, the discussed main types of metallopolymers-based DDSs involve metal complexes based on synthetic and natural polymers, coordination polymers, MOFs and metallodendrimers.

\subsection{Metal-containing synthetic polymers}

In recent years, more and more attention has been paid to the use of synthetic polymers for the creation of metallopolymersbased DDSs. These DDSs include linear or branched polymer chain that works either as the bioactive molecule, e.g., polymeric drug or as the inert carrier to which a drug can be covalently linked. A wide variety of synthetic polymers have been used as carriers for metallodrugs, especially biodegradable synthetic polymers, which mainly include polyesters, polypeptides and recombinant protein-based polymers. Typically, two different approaches are used to attach these metallodrugs to polymers: using polymers as a stable ligand for metallodrugs or the use of polymers as a leaving group of a metallodrug. At the same time, due to the lack of sufficient hydrophobicity or hydrophilicity, MPs are usually not good candidates for encapsulating organic drug.

As a typical example, we note the use of polymeric "ruthenium-cyclopentadienyl" conjugates bearing an organometallic fragment and polylactide chains as potential anti-cancer agents with proven cytotoxic activity ${ }^{13,134}$ and adequate aqueous stability. ${ }^{134}$ In another interesting example, a poly(L-glutamic acid)-graft-methoxy poly(ethylene glycol) (PGA-g-mPEG) has been proposed as a has been for inclusion of cisplatin. ${ }^{135,136}$ The resulting complex showed prolonged blood circulation time
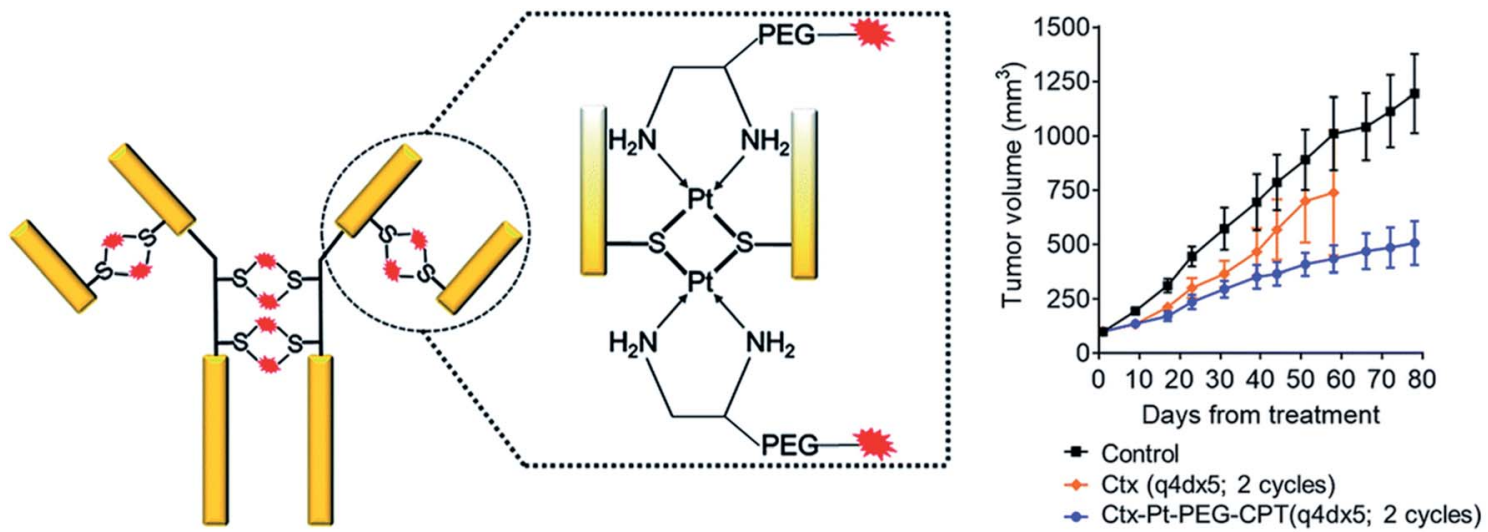

Fig. 9 (left) Pt(II)-linker re-bridges the antibody chains with a strong Pt-S interaction, giving stability, homogeneity and site-specificity. (right) In vivo efficacy in animals treated with Ctx-Pt-PEG-CPT (Ctx is cetuximab and CPT is camptothecin) showed significant differences in tumor volume compared with the control group $(P<0.016)$, with TGl of 55\% [reprinted from ref. 148 with permission from The Royal Society of Chemistry]. 


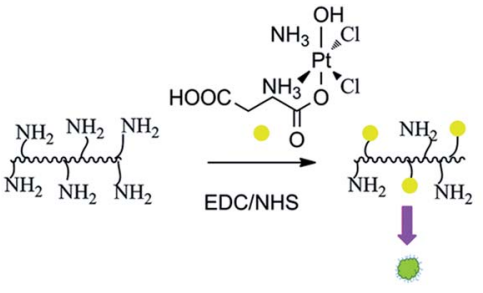

HA-EDA

HA-EDA-Pt(IV) nanoconjugate

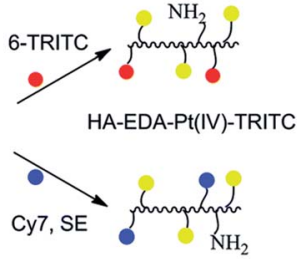

HA-EDA-Pt(IV)-Cy7, SE
Fig. 10 Synthetic pathways to the HA-EDA-Pt(IV) nanoconjugate, HAEDA-Pt(IV)-TRITC, and HA-EDA-Pt(IV)-Cy7, SE [reprinted from ref. 151 with permission from The Royal Society of Chemistry].

and excellent tumor accumulation. In particular, its plasma area-under-curve (AUC) was 31 times higher than that of free cisplatin, and its AUC in Lewis lung carcinoma tumors was 9.4 times higher.
Promising DDSs are metallopolymers based on polymer micelles. ${ }^{137,138}$ In particular, covalent attachment of a drug directly to a micelle is of interest to avoid its premature release in the circulation. So, poly(ethylene glycol)-block-PGA (PEG- $b$ PGA) was used as leaving group of the Pt(II) drugs of cisplatin and oxaliplatin. ${ }^{139}$ These MPs showed especially high in vivo micellar stability, reduced nephrotoxicity, increased accumulation in neoplastic tissues and reached a level of efficacy comparable to cisplatin. It should also be noted the use of polymer micelles for the delivery of $\mathrm{Pt}(\mathrm{II})$ and $\mathrm{Ru}$ (II) complexes to cancer cells. ${ }^{\mathbf{1 4 0}}$

Palladium catalysts were used to catalyze intracellular reactions by uncaging various model prodrugs, including the doxorubicin (DOX) prodrug (pro-DOX), for the effective tumor treatment in vivo. ${ }^{\mathbf{1 4 1}}$ The $\mathrm{Pd}$ (II) catalysts were encapsulated in the micellar core of diblock amphiphilic copolymers poly(lactic acid-co-glycolic acid) (PLGA)-PEG, which were intravenously delivered to various tumor cell models using the enhanced permeability and retention (EPR) effect. In tumor cells,

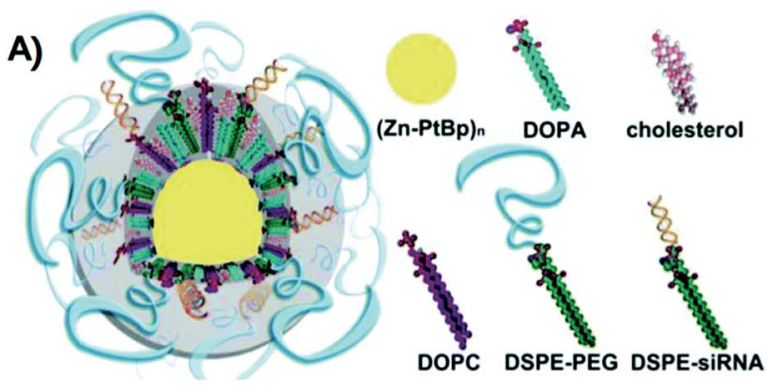

B)
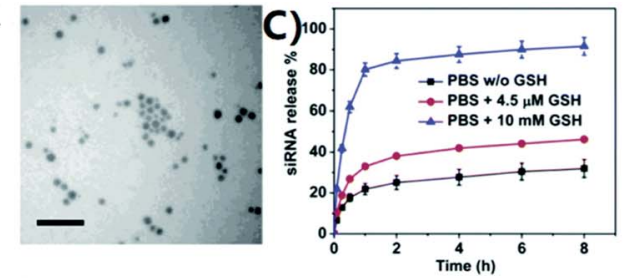

D)

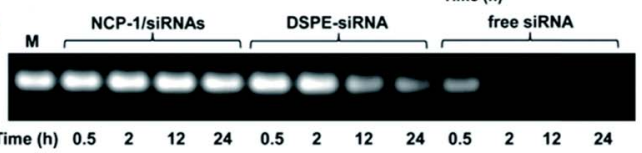

Fig. 11 Preparation and characterization of CP-1/siRNAs. (A) Schematic representation of CP-1/siRNAs carrying cisplatin in the solid core and siRNAs in the lipid shell. (B) Transmission electron microscopy (TEM) image of CP-1/siRNAs showing the spherical and monodispersed nanostructure. Bar $=100 \mathrm{~nm}$. (C) siRNA release from CP-1/siRNAs in the presence or absence of reducing agents. (D) Improved siRNA stability in serum of CP-1/siRNAs compared to free siRNA and DSPE-siRNA conjugate as evaluated by electrophoresis ( $2 \%$ agarose gel). "M" stands for untreated siRNA marker [reprinted with permission from ref. 154. Copyright 2016, American Chemical Society].
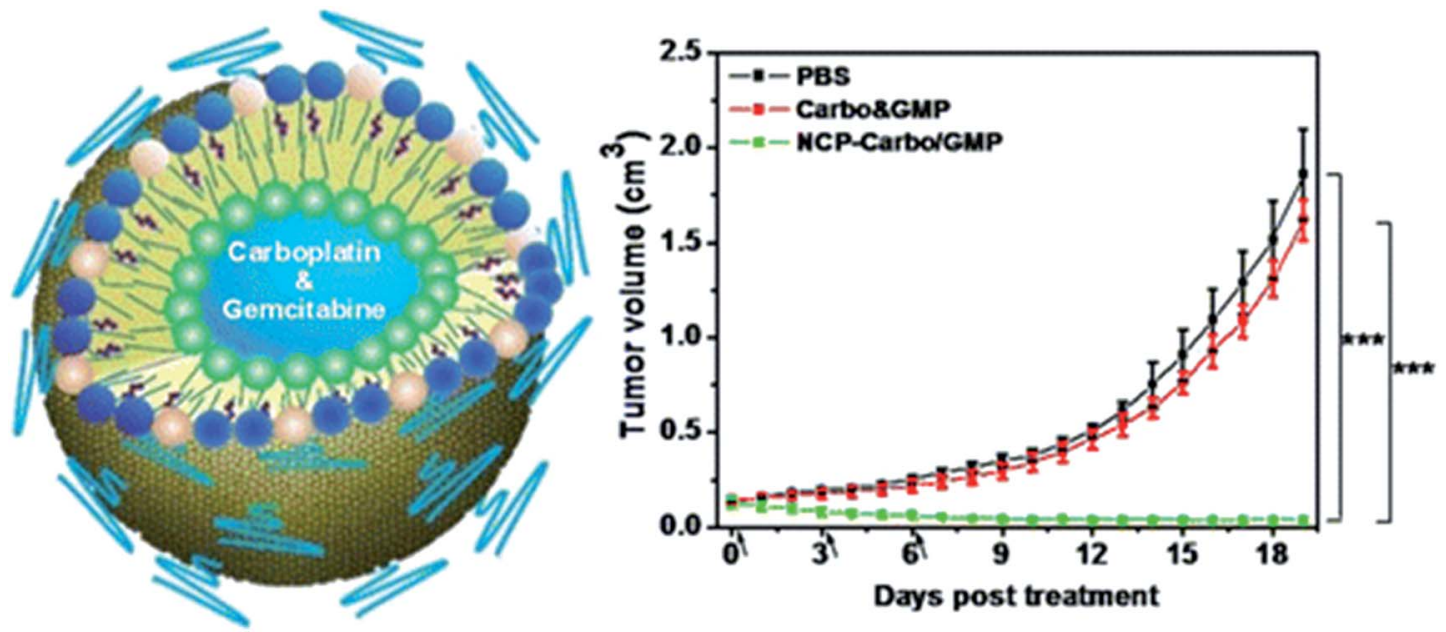

Fig. 12 (left) Schematic of CP-Carbo/GMP. (right) TGl in vivo. Carbo (dose, $10 \mathrm{mg} \mathrm{kg}^{-1}$ ) and GMP (4.6 mg kg ${ }^{-1}$ ) and CP-Carbo/GMP (doses, $10 \mathrm{mg} \mathrm{kg}^{-1}$ and $4.6 \mathrm{mg} \mathrm{kg}^{-1}$ ) were administered on days 0,3 , and 6 [reprinted with permission from ref. 155. Copyright 2016, American Chemical Society]. 
delivered $\mathrm{Pd}(\mathrm{II})$ into micelles can catalyze the cleavage of the alloc group to uncage the pro-DOX to initiate regression of tumor growth and apoptosis (Fig. 5).

Self-assembling cyclic peptides were conjugated to a biocompatible polymer poly(2-hydroxypropyl methacrylamide) and functionalized using highly potent candidate organoiridium anticancer complexes. ${ }^{\mathbf{1 4 2}}$ The antiproliferative activity of the three compounds (free drug, loaded onto the polymer, and the conjugate) was determined against human ovarian fibroblasts (HOF), a model for healthy, non-cancerous cells, and compared to that against A2780 ovarian cancer cells (Fig. 6). It turned out that drug-loaded nanotubes showed comparable or increased toxicity towards human ovarian cancer cells compared to free drug. It is important that the drugbearing conjugates do not increase the uptake of iridium, but rather demonstrate a more effective mode of action.

The acid-responsive design of the $\mathrm{Pt}(\mathrm{Iv})$ prodrug conjugates and the hydrophobic segment of the mPEG- $b$-PLA diblock copolymer, where PLA is a poly(lactic acid), helps to stabilize blood circulation, while also triggering the release of the drug into acidic endolysomal compartments (Fig. 7). ${ }^{143}$

A localized dual-drug delivery device was developed by simultaneously incorporating the Pt(Iv) prodrug backboned micelles and dichloroacetate into the poly(vinyl alcohol) nanofibers. ${ }^{144}$ It should also be noted the type of metallodrug, which is based on the Pt(Iv) prodrug self-cross-linked nanostructure with extremely high cisplatin loading efficiency. ${ }^{\mathbf{1 4 5}}$

It should be noted that metal complexes based on synthetic polymers have several advantages as DDS, such as easier synthesis of large quantities, insignificant batch-to-batch differences, and countless possibilities for post-synthetic modifications to achieve the desired properties. In addition, they focus on: increasing the solubility and stability of the drug, optimizing the dosage of the drug, protecting the drug from physical or chemical degradation, increasing the bioavailability, improving the pharmacokinetic behavior of the drug, reducing the duration of treatment, ensuring controlled release of the drug, reducing toxicity to tissue health and transporting the drug to the specific target sites.

However, when using DDS based on metal complexes with synthetic polymers, there are a number of serious drawbacks. For example, all types of synthetic polymers have some level of heterogeneity, since each MP molecule differs depending on molecular weight, drug loading and subsequent conformation. In addition, the difficulties in synthesis and characterization increase as MPs become more complex (i.e., multifunctional nanomedicines). Proven methods for physico-chemical characterization should also be established to certify the quality of the reproducible product. There is still an under-assessed problem with the possible toxicity and side effects of the metallopolymers-based DDSs. Although they can be biodegradable, their degradation rate must be analyzed and toxicity profiles evaluated in various in vitro, ex vivo and in vivo models. The development and application of strict safety rules is necessary to create an effective and safe dosage form.

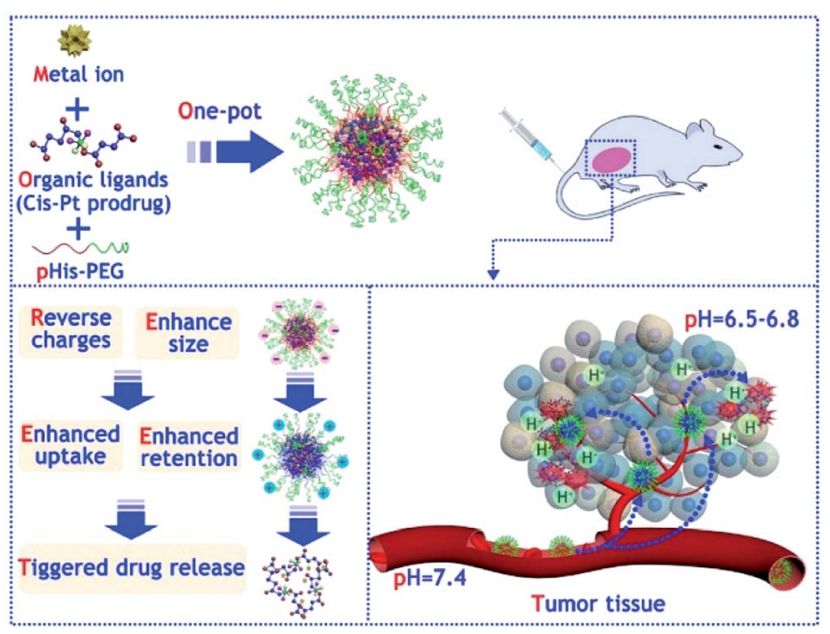

Fig. 13 Scheme illustrating the one-pot synthesis of PEGylated nanoscale CPs and their use for tumor microenvironment (TME)responsive anticancer therapy [reprinted with permission from ref. 156. Copyright 2018, Elsevier].

\subsection{Metal-containing natural polymers}

Natural biomacromolecules, such as proteins and peptides, are promising carriers for small metallodrug molecules due to their inherent biodegradability, biocompatibility, low toxicity and high solubility in water. It is also important that biological metallodrug carriers can recognize tumor markers overexpressed by cancer cells, which is easily used in targeted treatment methods. Finally, peptides and proteins are easy to manufacture and stable, which is often difficult for synthetic NPs. ${ }^{146}$

For example, the SV40 large $\mathrm{T}$ antigen-derived PKKKRKV peptide known as the nuclear localization sequence (NLS) peptide was used as a Pt(II) drug delivery vehicle (Fig. 8A). ${ }^{147}$ The construct efficiently translocated across the cellular membrane (Fig. 8B) and delivers platinum to the nucleus. The hybrid showed high biological activity in ovarian cancer cell lines, overcoming resistance to platinum, which may be associated with a higher $\mathrm{Pt}$ (II) content in the resistant cells. Quantitative determination of $\mathrm{Pt}$ (II) in the nucleus from two isogenic A2780 (Pt sensitive) and CP70 (Pt resistant) cells showed that in the first cells, one Pt(II) was attached to every 20th turn of DNA, while in the second cells every 10th turn.

Of interest are antibody drug conjugates (ADCs) with metallodrugs in which a Pt(II)-based linker can re-bridge interchain cysteines in an antibody (Fig. 9, left). ${ }^{148}$ ADC-Pt(II) constructs are more stable than conventional ADCs connected via maleimidebased chemistry, which reduces drug metabolism with blood serum albumin and off-target toxicity. ADC-Pt(II) is highly toxic in vitro, and in vivo studies using the A549 non-small cell lung cancer model showed marked tumor growth inhibition (TGI) with $\sim 80 \%$ of apoptotic cells in tumor sections (Fig. 9, right).

Natural polymers used in DDSs also include polysaccharides (agarose, hyaluronic acid (HA), ${ }^{\mathbf{1 4 9}}$ alginate, carrageenan, etc.) and protein-based polymers (collagen, albumin, gelatin, etc.). In particular, HA is a polyanionic polysaccharide that can be used 


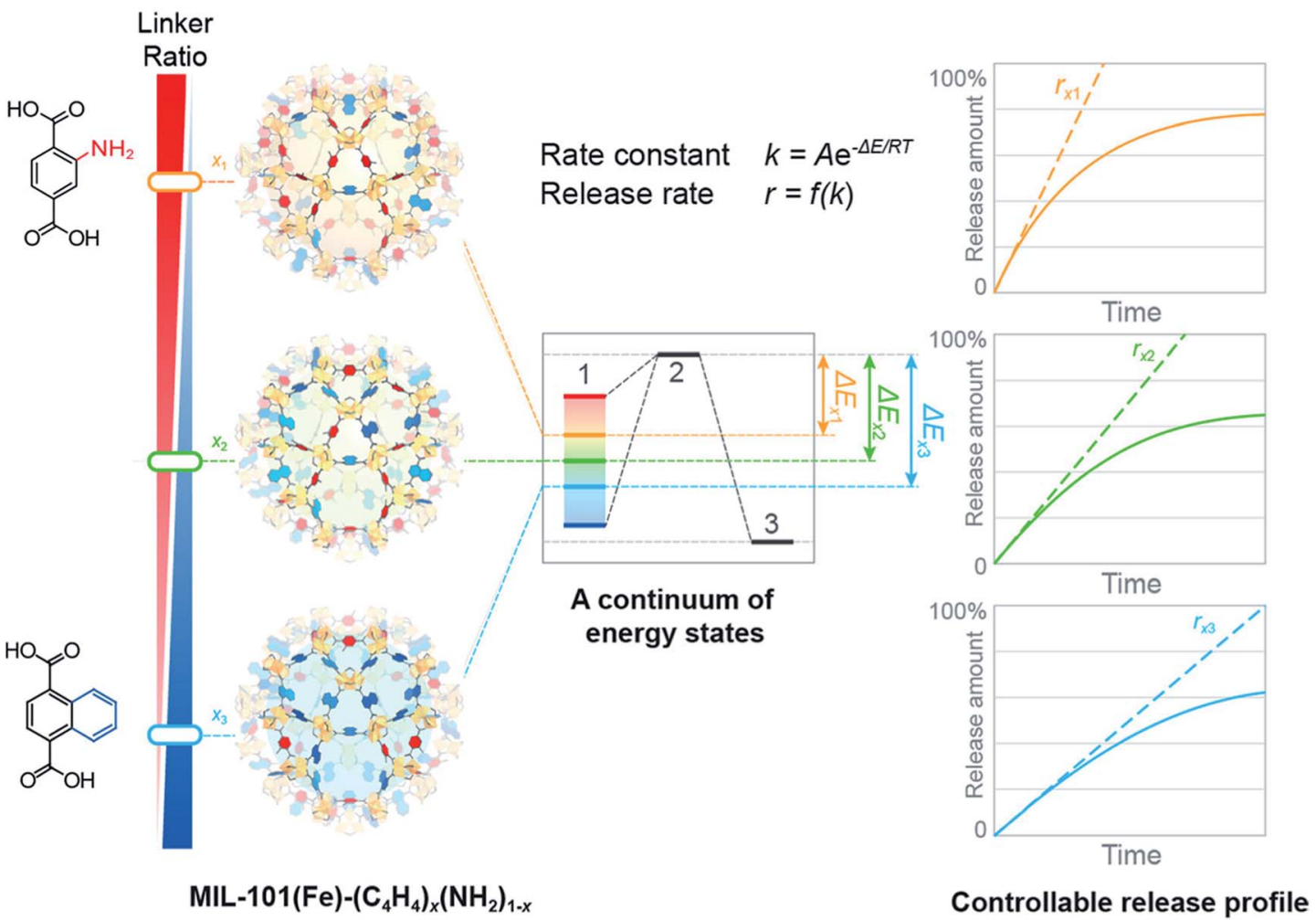

Fig. 14 Multivariate MOFs with various linker ratios present a series of continuous energy states from which guest release kinetics can be dialedin over a wide range [reprinted with permission from ref. 171. Copyright 2017, American Chemical Society].

both as DDS and as a target motif for drug delivery. ${ }^{150}$ Thus, the HA-Pt(IV) conjugate was obtained by attaching Pt(Iv) prodrugs to ethylenediamine (EDA)-modified HA (Fig. 10) via a succinamide bond (HA-EDA-Pt(Iv)). This complex was additionally labeled with fluorescent dyes for bio-imaging. The nanoconjugates accumulated in the tumor tissue and were selectively up taken by the tumor cells, which led to a decrease in systemic toxicity and prolongation of animal survival. ${ }^{151}$

A cisplatin templated hyaluronan-nanogel with targeting ability has been developed.$^{152}$ In the same way, cisplatin-bearing chondroitin sulfate-nanogels were obtained, which were then incorporated into $\mathrm{pH}^{-}$and temperature-responsive bioresorbable PEG-poly( $\beta$-aminoester urethane) hydrogels for the cancer cell-specific delivery of cisplatin. ${ }^{153}$

DDSs based on metal complexes with natural polymers have several advantages that allow you to control the release of drugs, avoid the use of hazardous organic solvents in the manufacture of samples, improve the pharmacokinetics and increase the biodistribution of therapeutic agents in target organs, which leads to increased efficiency, including a non-invasive drug delivery method. In addition, they have a wide molecular weight distribution, a variety of viscoelastic properties, biocompatibility, phase transition characteristics and an acceptable taste. Apart from advantages of DDSs based on metal complexes with natural polymers they have some disadvantages, for example, microbial contamination during production due to their natural sources, heavy metal contamination which is often associated with plant polymers, differences from batch to batch as a result of difference of resources and resource regions, slow production process, etc. These deficiencies depend on the environment and many other factors, and potential impurities can lead to unwanted immune reactions.

\subsection{Coordination polymers}

A significant number of studies are devoted to the use of coordination polymers as DDSs. As an example, we note longcirculating core-shell CPs, which effectively deliver multiple drugs with various mechanisms of action to enhance the synergistic therapeutic effects (Fig. 11). ${ }^{154}$ These CP particles contain high payloads of drugs cisplatin or cisplatin plus gemcitabine (GEM) in the core and pooled siRNAs that target multidrug resistant (MDR) genes in the shell. Even at low doses, intraperitoneal injections of NPs led to effective and prolonged tumor regression/eradication in subcutaneous and intraperitoneal xenograft mouse models of cisplatin-resistant ovarian cancer (OCa).

The use of a core-shell nanoscale metallopolymer CP-Carbo/ GMP, where Carbo is carboplatin and GMP is gemcitabine monophosphate, allows the delivery of high loads of Carbo (28.0 $\pm 2.6 \mathrm{wt} \%)$ and GMP $(8.6 \pm 1.5 \mathrm{wt} \%)$ (Fig. 12) ${ }^{155} \mathrm{~A}$ strong synergistic effect was observed between Carbo and GMP in relation to platinum-resistant OCa cells, SKOV-3 and A2780/ CDPP, in vitro. In addition, the use of this DDS led to an increase in the blood circulation half-life $(11.8 \pm 4.8 \mathrm{~h})$ and improved tumor uptake of drugs $\left(10.2 \pm 4.4 \% \mathrm{ID} \mathrm{g}^{-1}\right.$ at $\left.24 \mathrm{~h}\right)$, 


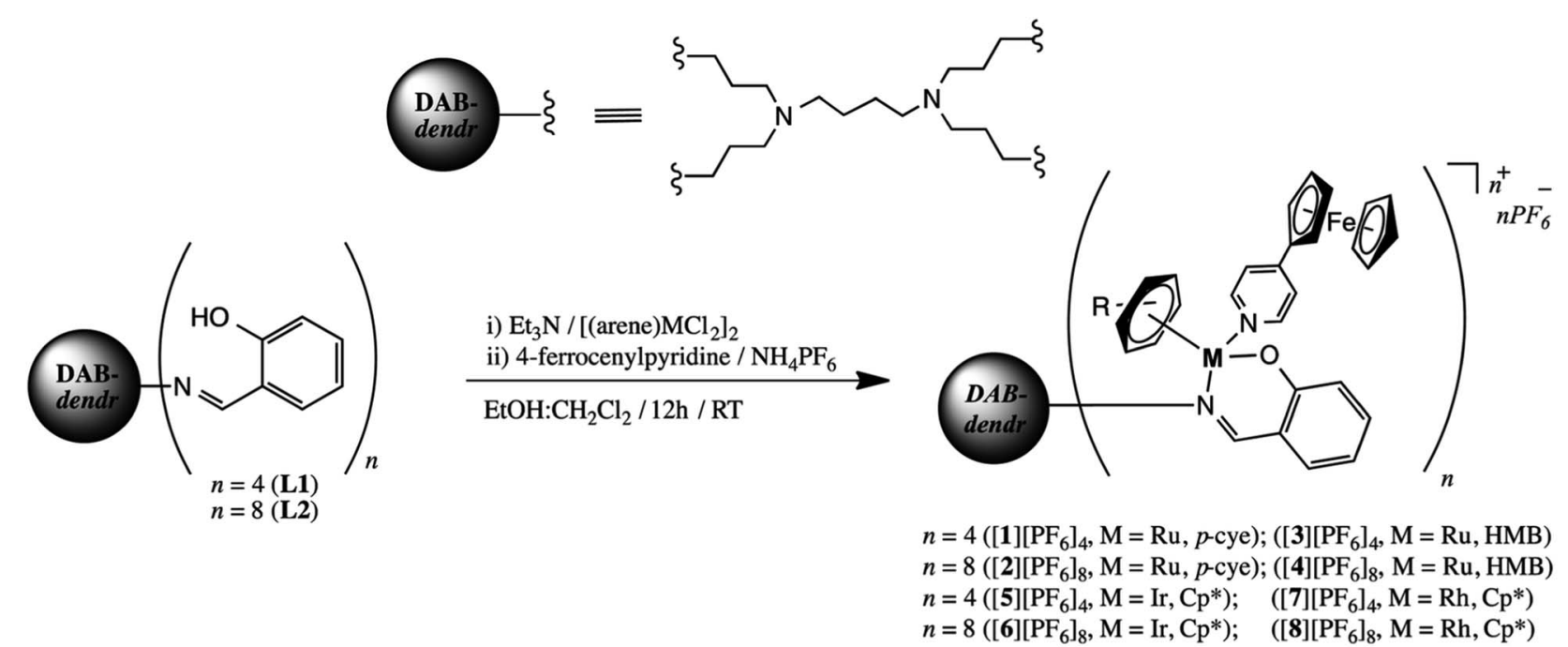

Scheme 1 Synthesis of ferrocenyl-derived heterometallic metallodendrimers $[1]\left[\mathrm{PF}_{6}\right]_{4}-[8]\left[P F_{6}\right]_{8}$ [reprinted from ref. 191 with permission from The Royal Society of Chemistry].

resulting in $71 \%$ regression and $80 \%$ TGI of SKOV-3 and A2780/ CDDP tumors, respectively.

PEG-modified CPs obtained by adding a polyhistidine (pHis)-PEG copolymer to a mixture of metal ions and organic ligands exhibit effective passive accumulation in the tumor, in which a reduced $\mathrm{pH}$ (about 6.5) will trigger charge conversion and size expansion to enhance their tumor retention and cell internationalization (Fig. 13). ${ }^{156}$ After cellular uptake of CPs in cell endo-/lysosomes, a further decrease in $\mathrm{pH}$ leads to the decomposition of these CPs and, consequently, to the release of the drug. Chemotherapy using CPs in an animal tumor model showed great efficacy at low doses of the drug and is especially effective against solid tumors with a reduced $\mathrm{pH}$.

Zinc CPs with the drug ibuprofen as a ligand have a high drug content and exhibit high stability, low cytotoxicity, good biodegradability and high biocompatibility. ${ }^{157}$ For the delivery of small-molecule chemotherapeutic agents, such as 5-fluorouracil (5-FU), one-dimensional (1D) zinc CP based on benzene1,3,5-tricarboxylic acid (trimesic acid) was used. ${ }^{158}$ The 5-FUloaded CP exhibited a time-dependent cytotoxicity effect against breast cancer cell lines MCF-7 and 4T1. The intratumor treatment with 5-FU-loaded $\mathrm{CP}$ showed favorable antitumor efficacy by inhibiting tumor growth.

Particular attention is drawn to nanoscale CPs that overcome many of the drawbacks of existing DDSs due to varying compositions, sizes and shapes, high drug loading, ease of surface modification and intrinsic biodegradability. ${ }^{\mathbf{1 1 1}} \mathrm{CPs}$, as an alternative to other nanocarriers, have many advantages due to the nature of the coordination polymer, such as high adaptability and diversity. In addition, CPs can combine the advantages of both organic polymer nanoparticles, such as compositional variability, biodegradability and high drug loading, and those of inorganic nanoparticles, such as welldefined particle morphologies and ease of surface functionalization, to create a completely new drug delivery platform.

\subsection{Metal-organic frameworks}

Over the past few years, MOFs with high surface area, large pore volume, and tunable composition have attracted much attention from researchers as DDSs. ${ }^{\mathbf{1 5 9 - 1 6 9}}$ For example, MOFs were functionalized with lipid bilayers for flexible encapsulation of drug molecules in the MOF cavities and used for smart drug delivery. ${ }^{170}$ Multivariate MOFs, in which multiple functional groups can be introduced into a single MOF crystal without changing the underlying topology, are of interest as DDDs for studying the interaction between MOF pores and drug molecules. ${ }^{171}$ In particular, a multivariate strategy provides access to a continuum of energy states that correspond to existing discrete energy levels specified by pristine MOFs, thereby adjusting the release profile of guest molecules accurately and over a wide range (Fig. 14).

MOF nanoparticles can be incorporated into exosomes to efficiently load and seal drugs and facilitate drug release through endogenous exosomal release and degradation of MOFs in the body. ${ }^{172}$ In addition, the resulting particles can be successfully loaded with one or several drugs, which makes liposome-coated MOF NPs interesting candidates for combination therapy. ${ }^{173}$ It is of interest to use MOFs as carriers for the delivery of chemotherapeutic drugs for the cancer therapy, such as 5-FU, DOX, CPT, platinum drugs and methotrexate (MTX). ${ }^{165,166,174-178}$ Thus, 5-FU-loaded MOFs exhibit excellent anticancer activity against human spinal cord tumor cells. ${ }^{179}$ DOX-loaded nano-sized MOFs (UiO-68) were modified with folic acid (FA) as a targeting agent and were used to effectively treat hepatoma by intravenous injection. Importantly, nanoscale MOFs have much higher anticancer efficacy in in vivo studies. ${ }^{\mathbf{1 8 0}}$

The zeolite imidazolate framework (ZIF-8) has been used as biocompatible MOFs for co-delivery of an anticancer drug (DOX) and a P-glycoprotein inhibitor of verapamil hydrochloride (VER) to overcome MDR and deliver an active targeting drug. The resulting design was further modified by PEG-FA to 

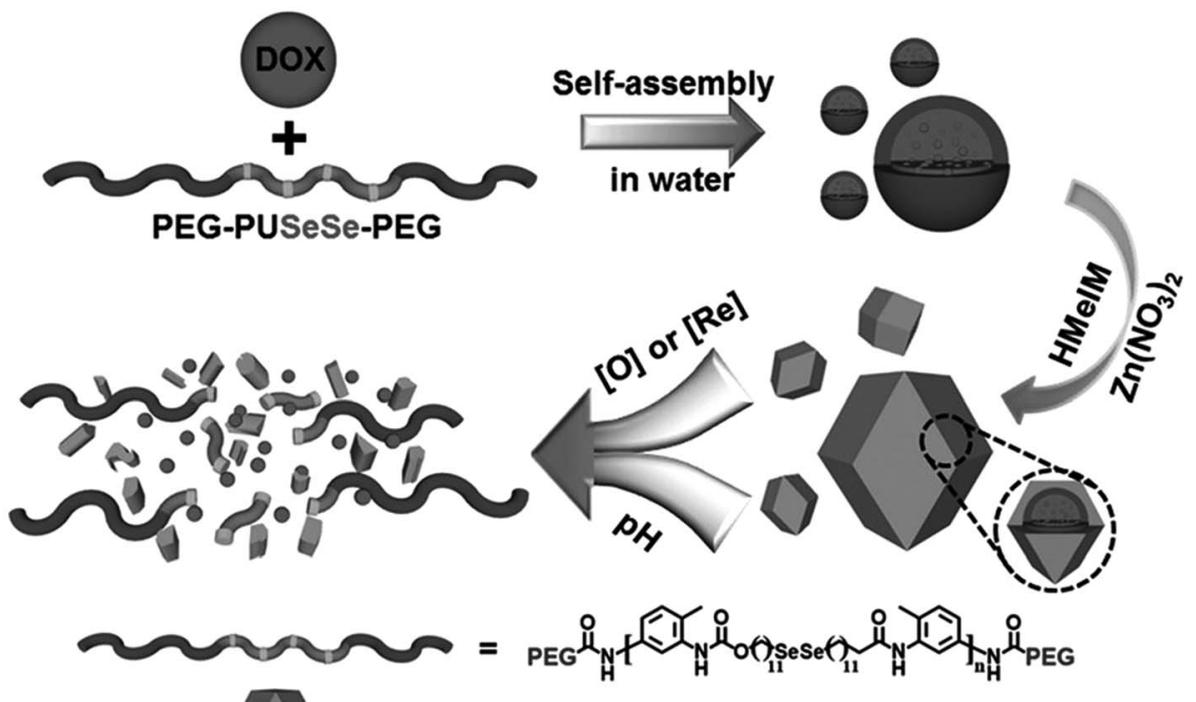

$=$ Metal-Organic Frameworks (MOFs)

Fig. 15 Schematic representation of the synthetic procedures of the PaZIF-8 nanocomposites as a DDS for the loading and multi-responsive release of DOX. HMelM: 2-methylimidazolate [reprinted with permission from ref. 198. Copyright 2017, John Wiley and Sons].

obtain better stability and active targeting ability. In particular, the multifunctional nanoplatform of PEG-FA/DOX-VER/ZIF-8 can efficiently overcome MDR in in vitro studies. ${ }^{181}$ Hollow MOFs microspheres with MOFs shells with inherent pores as windows and deep submicron-scale cavities were made in combination with FA on the surface of the microsphere as the targeting agent, which is a promising DDS for the cancer treatment. ${ }^{182}$

In addition, MOFs-based nanocomposites with gold nanoclusters were used to real-time monitor drug release through its external porosity and $\mathrm{pH}$-dependent luminescence. Composites demonstrated good biocompatibility and high luminescence for potential use in the cancer treatment. ${ }^{183}$

The superior properties of MOFs, such as distinct pore aperture, customizable composition and structure, adjustable size, versatile functionality, high drug loading, and improved biocompatibility, have made them promising candidates as DDSs. However, an important task is the manufacture of universal nontoxic or low-toxic and good biocompatible MOFs to ensure prolonged blood circulation and to ensure that degradation products can be processed through the body's metabolic system. In addition, systematic in vivo studies are needed to optimize MOF performance before clinical use.

\subsection{Metallodendrimers}

In recent years, a variety of multifunctional metallodendrimer systems has been used as DDSs. ${ }^{183}$ In particular, the inclusion of metallodrugs in dendritic frameworks can increase the activity and selectivity of these drugs. Indeed, metallodendrimers can combine the anticancer potential of metallodrugs with the features of dendrimers as nanocarriers and have been described as having promising cytotoxicity with respect to various cancer cell lines. Metallodendrimers, apparently, act according to a different mechanism of action compared to native metallodrugs, opening up new opportunities for searching for improved anti-cancer agents. ${ }^{184}$

Among the various dendrimers, polyamidoamine (PAMAM) dendrimers modified with PEG that are non-toxic, biocompatible, and non-immune reactions are most noteworthy. ${ }^{185}$ PEGylation of the dendrimer may provide more benefits, such as good transfection efficiency, tumor localization, better bioavailability, biodistribution, and pharmacokinetics. In the case of drug delivery efficiency, the amount of dendrimer generation is a major factor and can be well controlled. In addition, PAMAM dendrimers are soluble in aqueous solutions, have a large number of surface groups and a unique structure; thus, they are considered as a promising delivery system. The cytotoxicity of PEGylated G4 PAMAM dendrimer with physically incorporated Carbo on L929 fibroblasts demonstrated that the PEGylation of G4 can eliminate the toxicity caused by the amino groups of G4. ${ }^{186}$ The results of resazurin test and live/dead assay showed that the killing effect of G4-PEG@Carbo on three representative cells was achieved and increased after increasing the concentration of Carbo.

$\mathrm{Ru}(\mathrm{II})$ metallodendrimers containing arene fragments were evaluated as an antitumor nanotherapy against resistant prostate cancer. ${ }^{187,188}$ Promising cytotoxic, antiproliferative and antimetastatic behavior was observed. In particular, after treatment with these nanocompounds, the tumor volume of the mouse was significantly reduced compared with untreated animals. In addition, carbosilane metallodendrimers based on arene $\mathrm{Ru}(\mathrm{II})$ complexes showed relevant in vitro cytotoxic activity against a number of cisplatin-resistant cancer cell lines in the low micromolar range and low hemotoxicity. ${ }^{189}$ In general, dendritic polymers showed stronger cytotoxicity than their mononuclear analogues, which emphasizes the effect of the 


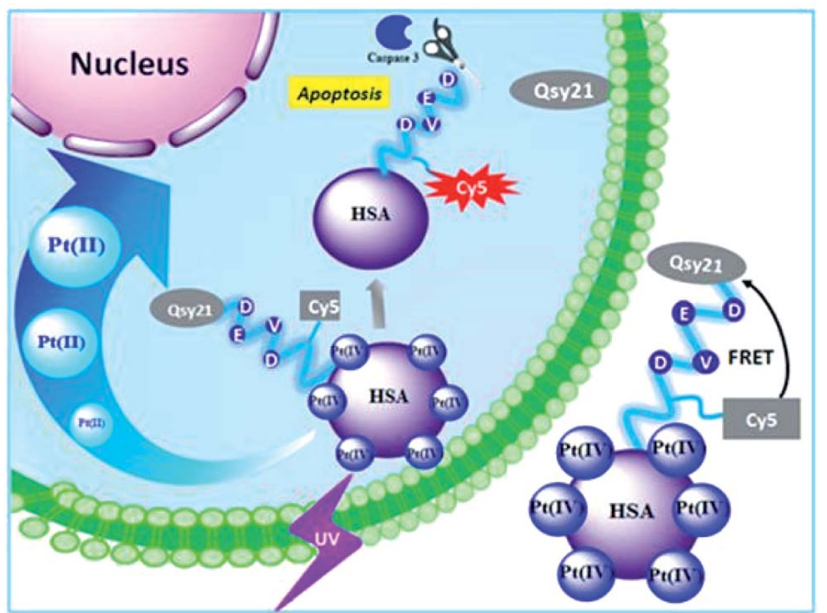

Fig. 16 Human transport protein carrier for the controlled photoactivation of an antitumor prodrug and real-time imaging of an intracellular tumor [reprinted with permission from ref. 210. Copyright 2015, American Chemical Society].

dendritic structure on antitumor activity. Interestingly, the G1 metallodendrimers have significant inhibitory properties against cathepsin-B, the protease is overexpressed in invasive and metastatic processes. Low generation dendrimers modified with an organometallic compound of $\mathrm{Ru}(\mathrm{II})$ showed high biological activity in vitro against various cancer cell lines, such as Caco-2 (colon adenocarcinoma), CAL-72 (osteocarcoma), and MCF-7 (breast cancer). ${ }^{190}$ Diaminobutane (DAB) dendrimers containing $\mathrm{Ru}(\mathrm{II})$-p-cymene, $\mathrm{Ru}(\mathrm{II})$-hexamethylbenzene, $\mathrm{Rh}(\mathrm{III})$ cyclopentadienyl, and Ir(III)-cyclopentadienyl fragments exhibited cytotoxicity, but the most pronounced effects were observed for the ferrocenyl-derived $\mathrm{Ru}(\mathrm{II})$-hexamethylbenzene metallodendrimer (Scheme 1) ${ }^{191}$ It is important to note that all metallodendrimers showed lower cytotoxicity towards noncancerous cells.
Hyperbranched polyglycerol (hPG) was used as a multivalent framework to develop nanocarriers capable of high-affinity encapsulating copper for treatment of various pathological conditions associated with Cu-deficiency. ${ }^{192}$ Cu-binding domains were attached exclusively in the hPG core by selective differentiation between the primary and secondary hydroxyl groups of the polyol. These $\mathrm{Cu}$-containing nanocomplexes showed a moderate cationic surface charge, which led to controlled protein binding characteristics and a low cellular toxicity profile.

Macromolecular, multivalent, Cu-encapsulating nanocarrier platforms based on hPG have been developed for therapeutic use. ${ }^{193}$ Copper-containing hPG with EDA fragments can cross the artificial blood-brain barrier (BBB). Cu(II)-renal NPs crossed the BBB model twice as effectively as ${ }^{14} \mathrm{C}$-sucrose and sodium fluorescein, and up to 60 times better than Evans Blue-labeled albumin. The NP core-shell design allowed the formation of the $\mathrm{Cu}(\mathrm{II})$ complex in the outer shell and, therefore, facilitated the $\mathrm{pH}$-dependent release of $\mathrm{Cu}$ in contrast to core-multishell NPs, where $\mathrm{Cu}$ (II) ions are encapsulated in the core. In addition, $\mathrm{Cu}$ can also be released intracellularly from NPs and is available for biological processes.

Thus, the range of MPs used as DDSs is quite wide and is not limited to the examples given here. A huge selection of MPs with a wide arsenal of synthetic methods for their preparation, the simplicity of many formulations, scalability and an acceptable price/quality ratio provide a methodological platform to creating promising DDSs based on them. In particular, one promising area is the use of bioactive motifs (cations, organic ligands, or both) as building blocks for constructing metal biomolecule frameworks (also known as bioMOFs). ${ }^{194}$ For example, biomolecules, such as amino acids, peptides, and nucleic bases, as well as biocompatible metal cations (Fe, Zn, and $\mathrm{Cu}$ ), were used as ligands in the construction of this MOF subclass. ${ }^{195,196}$ A variety of hybrid materials, such as biopolymer@MOFs, are also of considerable interest. ${ }^{197}$ It should

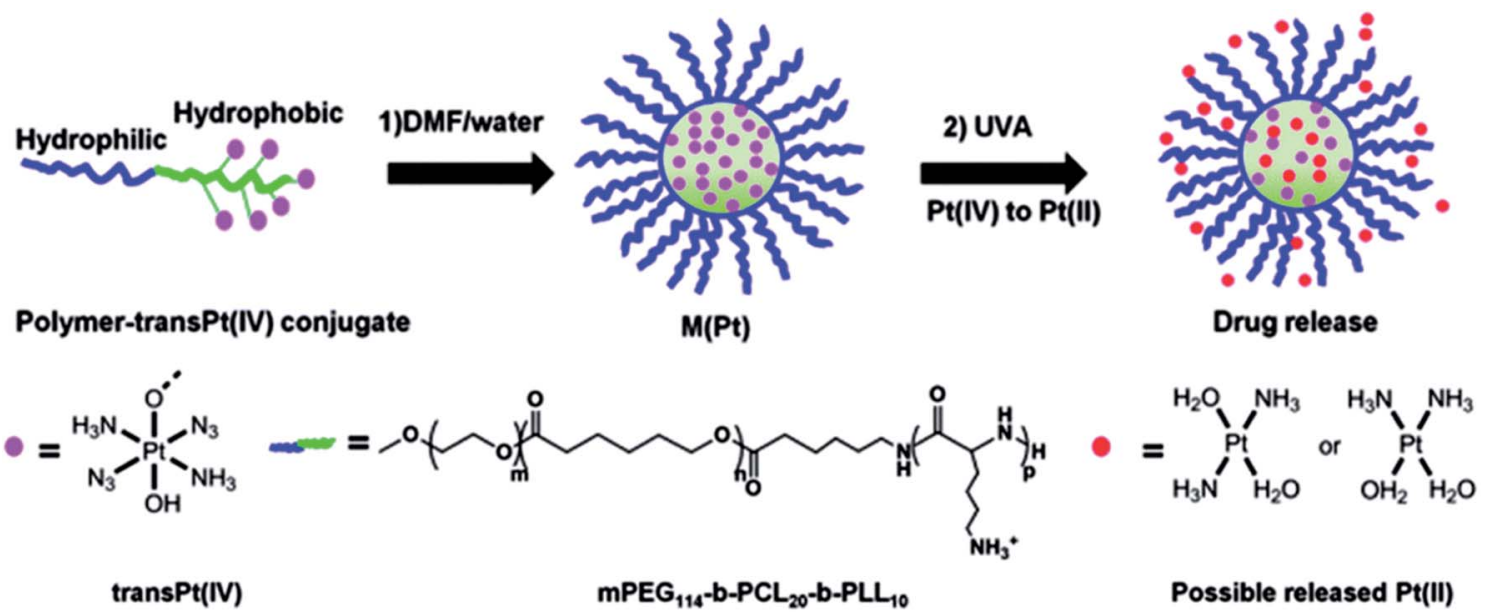

Fig. 17 Attachment of trans-Pt(Iv) to the $\mathrm{mPEG}_{114}-b-\mathrm{PCL}_{20}-\mathrm{PLL}_{10}$ copolymer and self-assembly of the polymer-trans $\mathrm{Pt}(\mathrm{IV})$ conjugate into micelles $(\mathrm{M}(\mathrm{Pt})$ ). After UVA irradiation, $\mathrm{Pt}(\mathrm{IV})$ prodrugs are rapidly reduced to $\mathrm{Pt}(\mathrm{I})$ and released [reprinted from ref. 214 with permission from The Royal Society of Chemistry]. 
a)

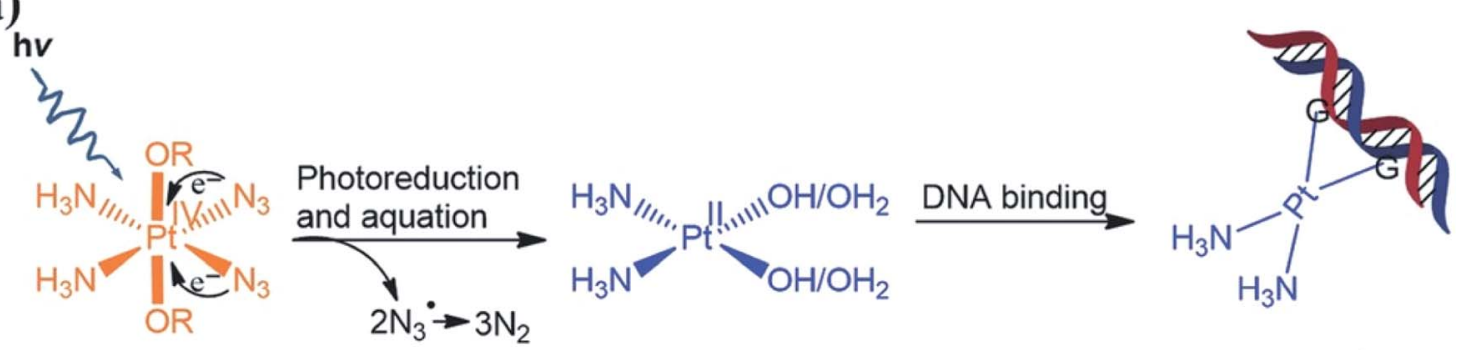

$\mathrm{Pt}(\mathrm{IV})$-azide complex

Pt(II) complex

Cell death

$R=H$, prodrug
$R=$ polymer chain, prodrug-backboned polymer

b)

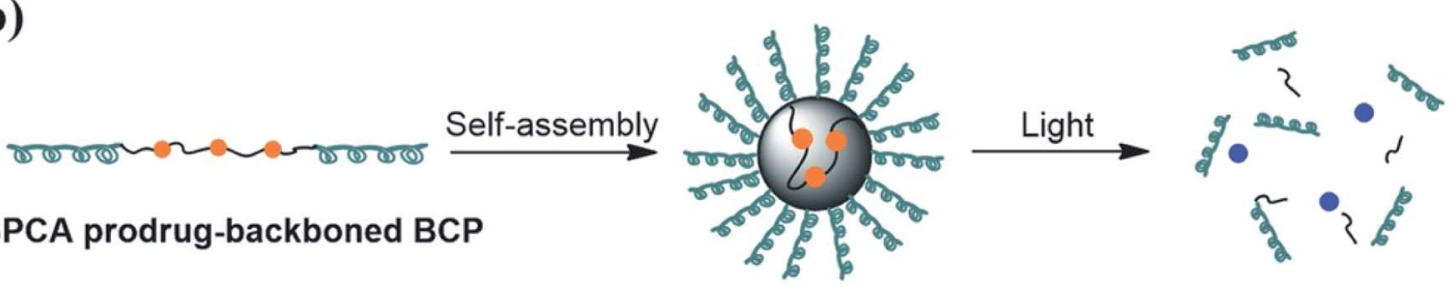

- Photo-responsive prodrug - Active drug

Fig. 18 Schematic representation of (a) the anticancer mechanism of photo-responsive Pt(Iv)-azide complexes and (b) self-assembly and light-triggered dissociation of simultaneously photo-cleavable and activatable (SPCA) prodrug-backboned BCP micelle [reprinted with permission from ref. 215. Copyright 2016, John Wiley and Sons].
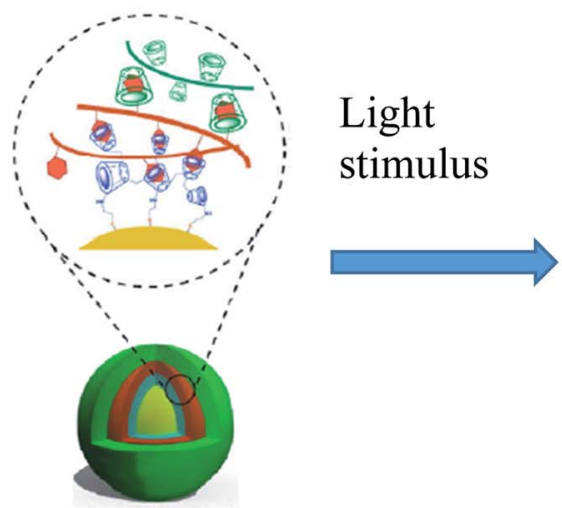

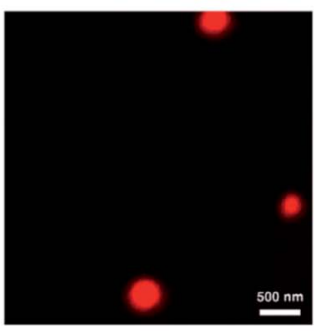

Encapsulation

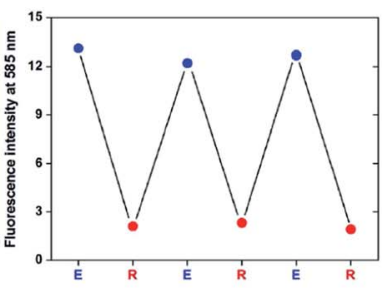

E: encapsulation

$\mathrm{R}$ : release

Fig. 19 Encapsulation of RhB after the incubation with NCs after the UV-light irradiation and variations in the fluorescence intensity at $585 \mathrm{~nm}$ for three sequential encapsulation/release cycles [reprinted with permission from ref. 219. Copyright 2016, Springer Nature].

be noted the use of MPs whose polymeric chains include nontraditional heteroatoms, for example, selenium. Thus, selenium-containing polymer (P) micelles were obtained with the drug encapsulated in MOFs (Fig. 15). ${ }^{198}$ In the presence of external redox agents and $\mathrm{pH}$ stimuli, the prepared nanocomposite (P@ZIF-8) drug system showed DOX release ability and good selectivity in DOX release at low $\mathrm{pH}$ instead of normal $\mathrm{pH}$.

Dendrimer-based DDSs and dendrimers as drugs alone are the two main therapeutic applications of dendrimers. ${ }^{199}$ A number of significant advantages of dendrimers in drug delivery should be noted: ${ }^{\mathbf{2 0 0}-204}$ three-dimensional architecture, high biocompatibility, good solubility in water, biomimicry, controlled sizes, monodispersity, easy modification, reducing the toxicity of the drug itself, extending the route of administration, improvement of the disease outcomes of drugs, improvement of the pharmacokinetic/pharmacodynamic profile of drugs, etc. Dendrimers are excellent nanocarriers for various cargoes, such as drugs and metallodrugs, increasing the selectivity, specificity, stability and biological activity of drugs. ${ }^{205}$ Interaction of a dendrimer with a drug or drug loading can be achieved in several ways: (1) encapsulation inside the dendrimer; (2) electrostatic encapsulation; (3) covalent conjugation. Currently, interest in metallodrug-dendrimer 

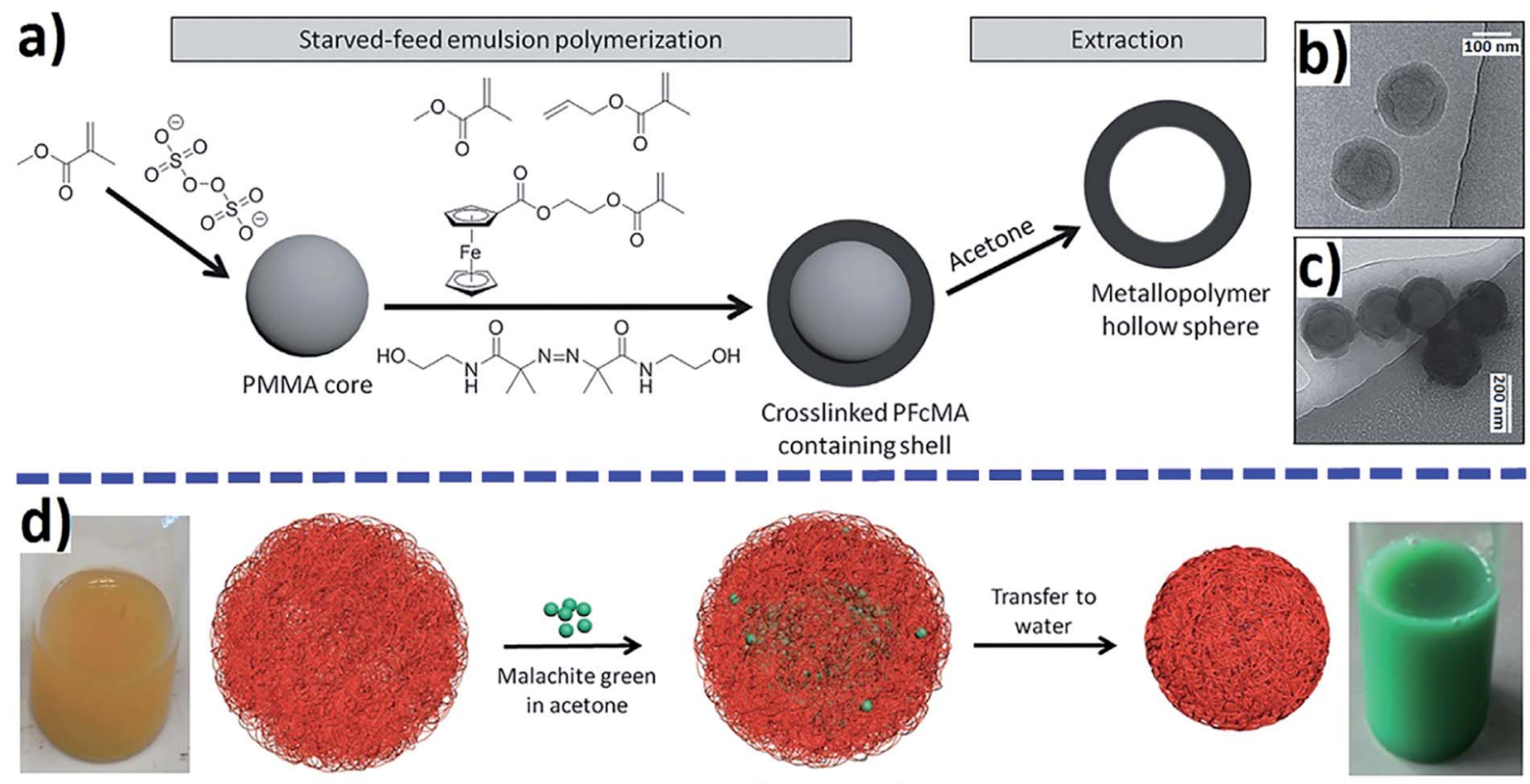

Swollen capsule

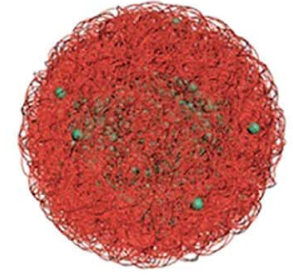

Swollen capsule with malachite green in acetone

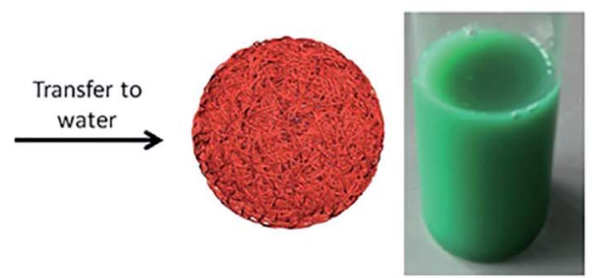

Sealed capsule loaded with malachite green

Fig. 20 Preparation of PFCMA-containing NCs by sequential starved-feed emulsion polymerization and subsequent extraction (a) and loading malachite green as a model drug (d). Cryo-TEM images of PFcMA capsules (b) and oxidized PFcMA capsules (c) [reprinted with permission from ref. 223. Copyright 2016, John Wiley and Sons].

conjugates and encapsulation of metallodrugs in dendrimers has increased significantly, in particular with the aim of reducing toxicity, increasing bioavailability and overcoming resistance. It should be noted that an undeniable advantage of metallodendrimers as DDSs is the possibility of their functionalization by varied ligands to achieve tumor tissue through various body barriers in the body with minimal loss of activity in the bloodstream. In addition, they have the ability to selectively kill tumor cells without affecting normal cells, and, most importantly, with an actively controlled release mechanism. The disadvantages of metallodendrimers include insufficient drug carrying capacity, biodegradability, toxicity, characteristics of biodistribution and favorable retention with appropriate specificity and bioavailability.

\section{Stimuli-responsive metallopolymers-based drug delivery systems}

Recently, interest in stimuli-responsive polymers has increased significantly, especially in their applications as DDSs. ${ }^{24,25,206}$ In the field of DDSs, stimuli-responsive polymers have attracted great attention due to their unique properties, including the enhanced solubility of the drug molecules, the EPR effect, and most importantly, the effective control of chemical and physical characteristics with respect to the field of applications. Over the past two decades, a variety of metallopolymers-based DDSs has been produced that respond to therapeutic stimuli, such as redox, pH, enzyme, light, temperature, etc. ${ }^{98,207}$ They are divided into three main categories based on the nature of the stimuli, namely: endo-stimuli-responsive DDSs, exo-stimuli-responsive DDSs, and dual-stimuli-responsive DDSs.

\subsection{Photo-responsive DDSs}

Among the many external stimuli, the light trigger strategy attracts more and more attention due to its usefulness for ondemand therapy in well-defined sites that arise due to the non-invasive and spatiotemporal exact mode of action after light irradiation. As an example, we note the integration of COreleasing complexes and polymers as one of the promising

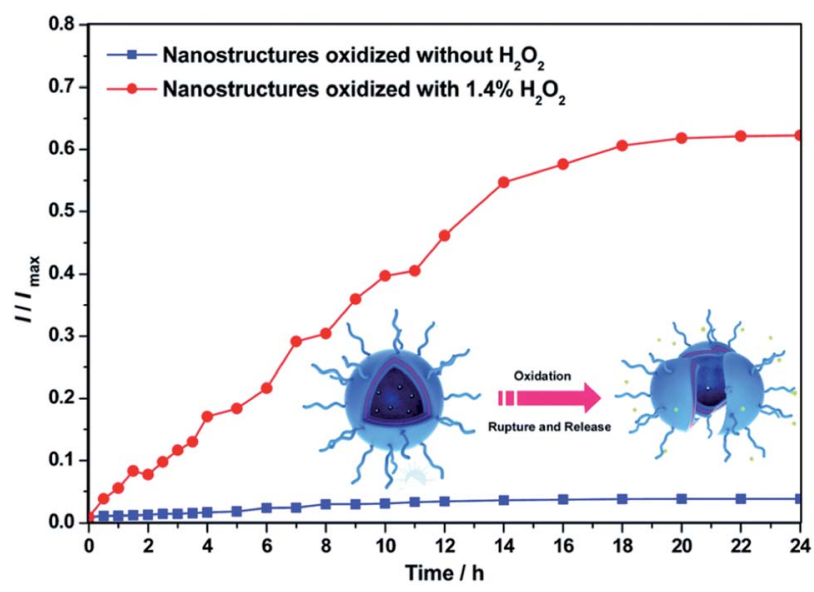

Fig. 21 Release kinetics of RhB from Fc-containing amphiphilic BCP. The fluorescence intensity of the oxidized vesicles increases with time, indicating a redox release of $\mathrm{RhB}$ [reprinted from ref. 224 with permission from The Royal Society of Chemistry]. 

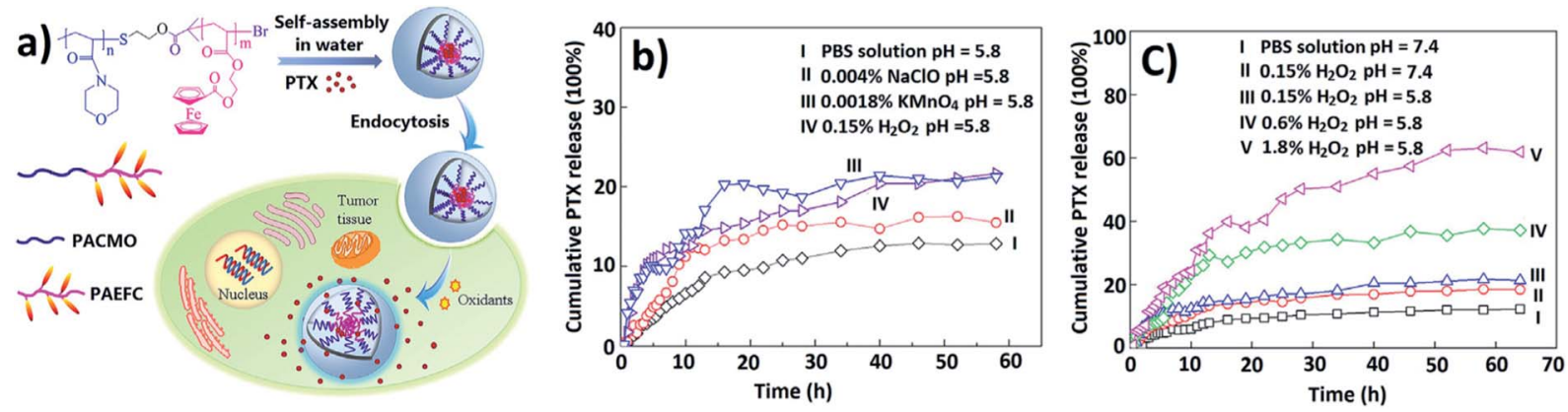

Fig. 22 (a) In vitro loading and release chart of a drug from $\mathrm{PACMO}_{95}-b-\mathrm{PAEFC}_{25}$ micelles when triggered by oxidative stimuli. Cumulative release of PTX from PACMO $_{95}-b-P A E F C_{25}$ micelles triggered by various oxidizing agents (b) and different concentrations of $\mathrm{H}_{2} \mathrm{O}_{2}(\mathrm{c})$ [reprinted with permission from ref. 225. Copyright 2017, American Chemical Society].
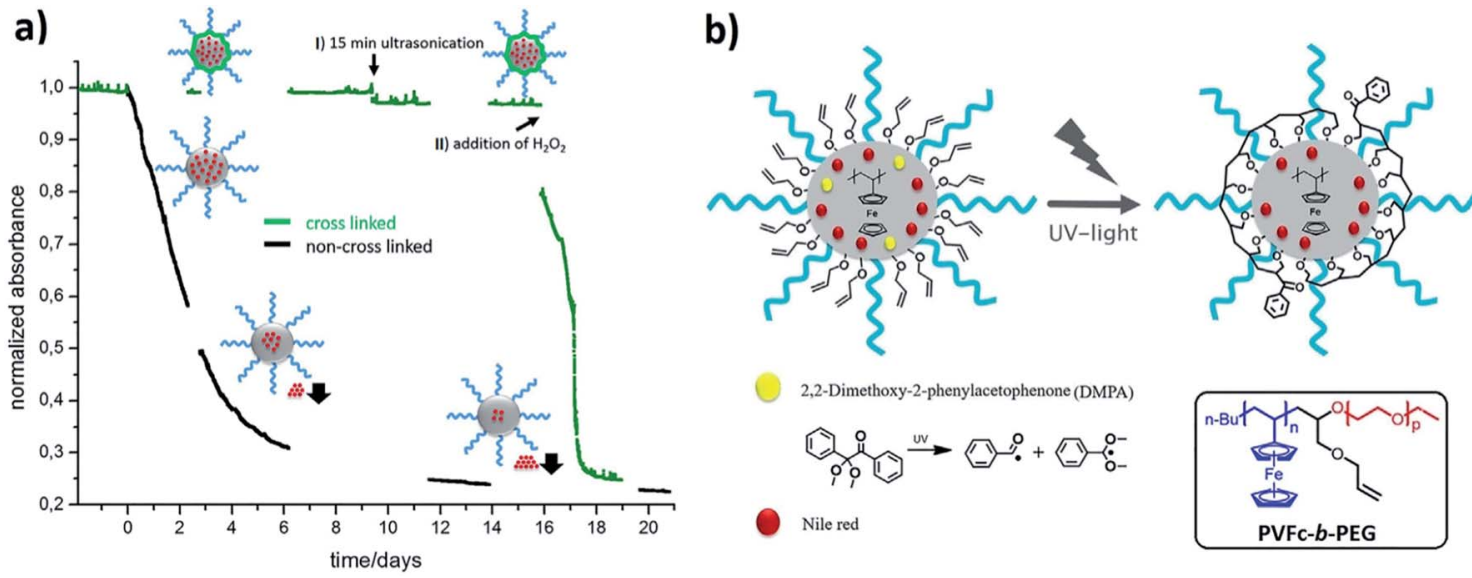

Fig. 23 Schematic cross-linking of PVFc- $b$-PEG micelles and its effect on the stabilization of encapsulated NR. (a) UV-vis measurement of the release of encapsulated NR. Black: uncross-linked micelles, decrease of absorbance due to uncontrolled NR release. Green: cross-linked micelles, no uncontrolled release: (I) no release through ultrasonication; (II) release after oxidation. (b) Scheme of the stage of crosslinking of inserted allyl groups in the corona of PVFc cores. The reaction scheme for the dissociation of a radical photoinitiator into an active benzoyl radical and an inactive acetal radical [reprinted with permission from ref. 226. Copyright 2016, American Chemical Society].

strategies for achieving localized on-demand delivery of $\mathrm{CO}$ for therapeutic purposes. ${ }^{208}$ In particular, a photoactive COreleasing protein composition has been developed for doseregulated delivery in living cells. ${ }^{209}$ Cellular uptake and lightinduced (e.g., $456 \mathrm{~nm}$ light) CO-releasing properties of this protein cage activate nuclear factor- $\mathrm{kB}$ and tumor necrosis factor an in mammalian cells.

In another interesting example, a human serum albumin (HSA) protein-based nanocarrier was synthesized, which combined the photoactivatable Pt(Iv) antitumor prodrug for controlled release and a fluorescent light-up probe to evaluate the effect and effectiveness of the drug (Fig. 16). ${ }^{210}$ This platform is locally activated by UV irradiation to release active Pt species, which led to increased cell death in both drug-sensitive A2780 and cisplatin-resistant A2780cis cell lines compared to free prodrug molecules. Cytotoxicity leads to apoptosis by activation of caspase 3, a typical programmed cell death protease, which can be visualized using apoptosis-sensitive probes on the platform.

It should be also noted cisplatin DDS with high encapsulation efficiency (EE) and near-infrared (NIR) light stimuli- responsive drug release properties. ${ }^{211}$ Under the stimuli of a NIR laser, the hydrophobic block of the polymer became more hydrophilic, which led to the rapid release of cisplatin. Photosensitive Pt(Iv) prodrugs based on cisplatin and oxaliplatin were developed. ${ }^{212}$ An example is the triblock polymer mPEG- $b$ poly( $\varepsilon$-caprolactone)- $b$-poly-L-lysine, $\mathrm{mPEG}_{114}-b-\mathrm{PCL}_{20}-b$ - $\mathrm{PLL}_{10}$, and the resulting the $\mathrm{Pt}(\mathrm{Iv})$ drugs can be activated by UV irradiation to release toxic $\mathrm{Pt}(\mathrm{II})$ species (Fig. 17). ${ }^{213,214}$ These photosensitive $\mathrm{Pt}(\mathrm{Iv})$ nanoformulations exhibit sharply increased cytotoxicity compared to clinically prescribed platinum drugs, reaching 8 times greater cytotoxic activity than cisplatin and 13 times higher than oxaliplatin. In addition, the inclusion of prodrugs in nanoformulation improves blood circulation; the half-life of a micelle in the blood exceeded $30 \mathrm{~min}$, which is more than 10 times higher compared to molecular prodrug.

A simultaneously photo-cleavable and activated prodrugbackboned block copolymer (BCP) micelle was developed using an anticancer prodrug, trans,trans,trans- $\left[\mathrm{Pt}\left(\mathrm{N}_{3}\right)_{2}(-\right.$ $\mathrm{OH})_{2}$ (py $\left.)_{2}\right]$ (Fig. 18). ${ }^{215}$ Concurrent chain cleavage of the micelle and the activation of the $\mathrm{Pt}(\mathrm{Iv})$ prodrug can be temporally and 


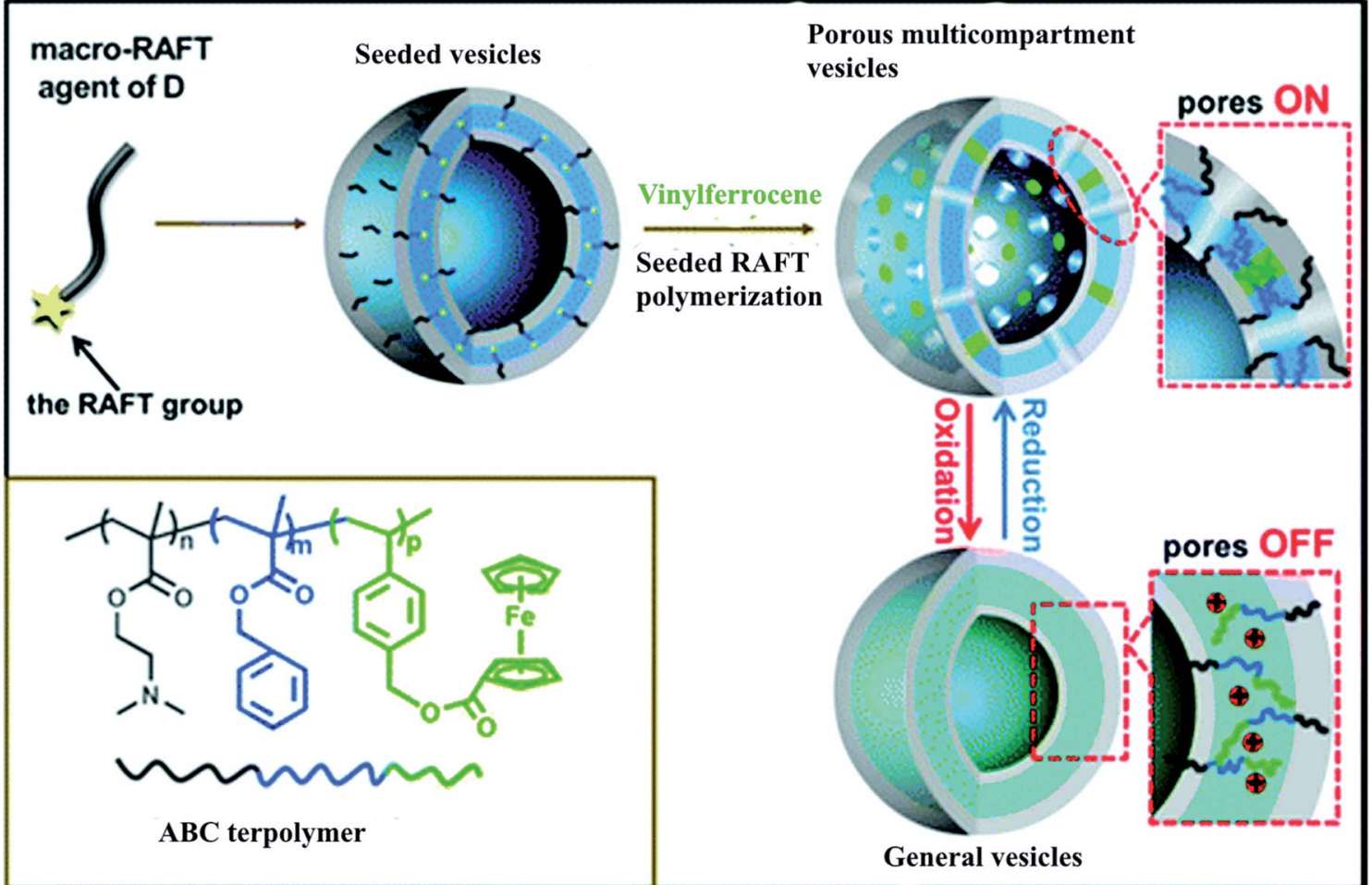

Fig. 24 Synthesis of the porous multicompartment vesicles of ABC triblock terpolymer by seeded RAFT polymerization and the schematic onoff switch of the membrane pores of the multicompartment vesicles through oxidation/reduction [reprinted with permission from ref. 227. Copyright 2016, American Chemical Society].

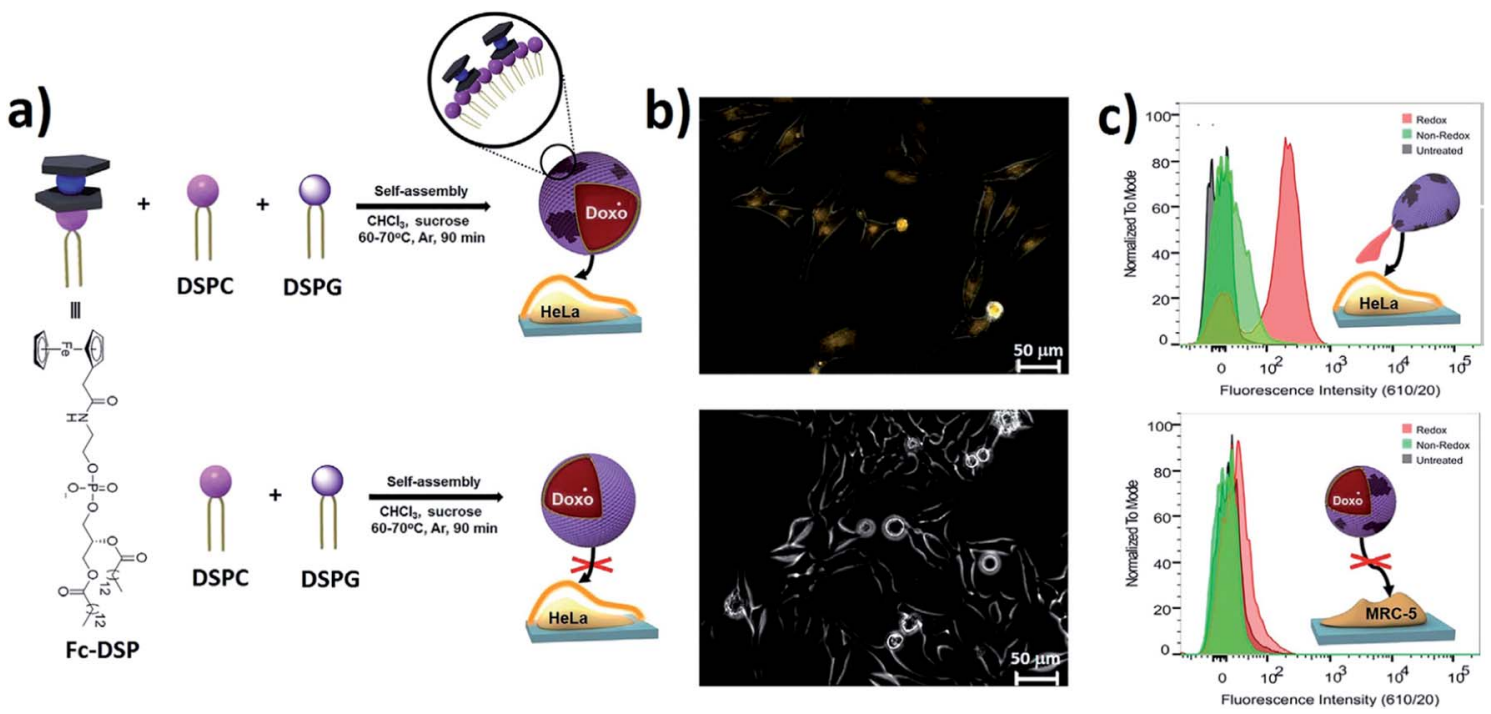

Fig. 25 Self-assembly formation of DDSs from Fc-DSP and selective release into cancer cells. (a) Schematic formation of the GUVs. (b) Fluorescence microscopy images of HeLa cells that were exposed to redox-active and non-redox-active GUVs. (c) Flow cytometry of HeLa and MRC -5 cells exposed to control (black), redox-active (red), and non-redox-active (green) active GUVs [reprinted with permission from ref. 228. Copyright 2016, American Chemical Society].

spatially triggered under the influence of UV or even visible light for accurate delivery of anticancer drug. When exposed to light, the micelle is about 1.5-4.0 times more cytotoxic against HeLa cells than in the dark, than and twice as effective as. Even micelles activated by visible light ( 430 and $500 \mathrm{~nm}$ ) showed an efficiency comparable to cisplatin.

One very promising strategy for drug encapsulation involves the formation of non-covalent host-guest complexes. ${ }^{216}$ In 


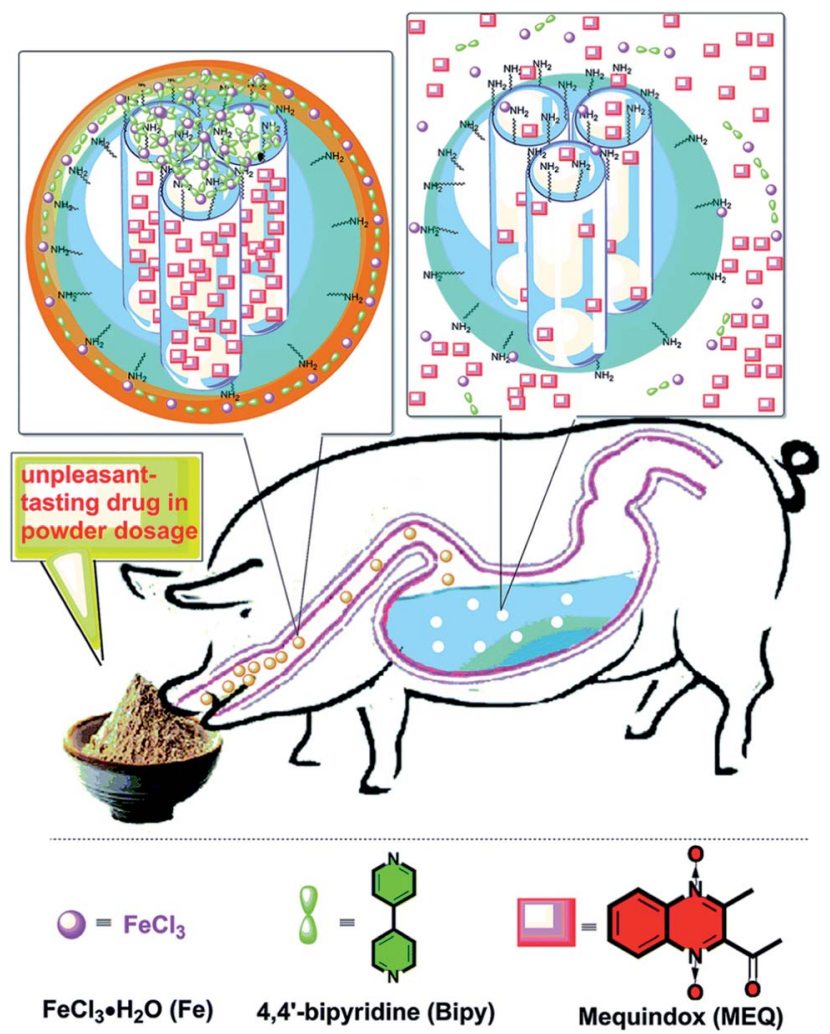

Fig. 26 Schematic representation of a working protocol for a tastemasking drug based on physiological $\mathrm{pH}$-responsive release of an unpleasant-tasting drug from MSNPs capped with the CP shell [reprinted from ref. 233 with permission from The Royal Society of Chemistry]. particular, due to the high binding constant of ferrocene $(\mathrm{Fc})$ units with $\beta$-cyclodextrin ( $\beta$-CD), a pathway has been developed for the delivery of various drugs and the use of smart materials. ${ }^{217}$ Fc-based photo-responsive materials have attracted much attention as an integral part of biological applications, such as drug delivery. ${ }^{218}$ An example is light-responsive hollow nanocapsules (NCs) based on dextran (Dex) with $\beta$-CD and Fc fragments, in which the light stimulus significantly increased the encapsulation process of rhodamine $\mathrm{B}(\mathrm{RhB})$ compared to the process without a stimulus (Fig. 19). ${ }^{219}$

\subsection{Redox-responsive DDSs}

The advantage of a redox stimulus is a change in composition without the addition of chemicals and is attracting increasing attention due to its light trigger mode and the significance of potential changes in biological systems. ${ }^{220,221}$ For example, the host-guest interaction between $\beta-\mathrm{CD}$ and $\mathrm{Fc}$ can respond to redox stimuli and is used in redox-responsive MPs. These supramolecular structures are widely used as DDSs. Thus, the substrate loading ability of the redox-responsive supramolecular Janus device based on $\beta$-CD and 2-fold Fc host-guest interactions was confirmed with $\mathrm{RhB}$, and the oxidation of the Fc groups led to the release of loaded cargos. ${ }^{222}$ The reversible ferrocene/ferricinium ( $\mathrm{Fc} / \mathrm{Fcium})$ redox couple has attracted considerable attention for the dynamic redox switching of DDSs created using Fc-containing polymers. ${ }^{116}$ The "on-demand" release of loaded drugs was achieved in response to external redox stimuli.

Breathing poly-2-(methacryloyloxy)ethylferrocenecarboxylate (PFcMA)-containing NCs were used for redox-controlled release
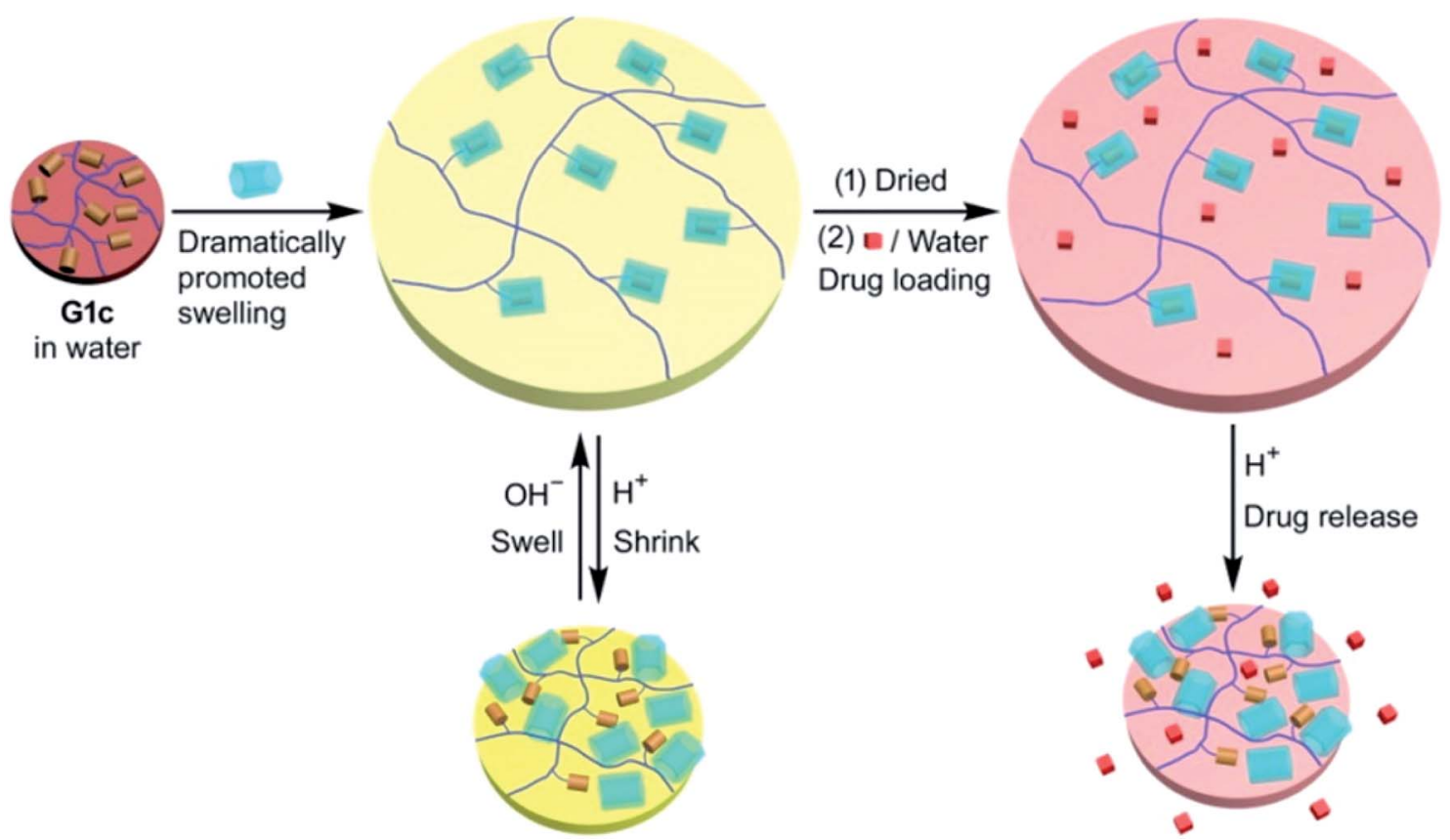

Fig. 27 Illustration of a $\mathrm{pH}$-responsive drug (DOX. $\mathrm{HCl}$ ) release from $\mathrm{G} 1 \mathrm{c} \cdot \mathrm{WP} 6$ with a loaded drug and corresponding profiles of in vitro cumulative drug release in an aqueous release medium at different $\mathrm{pH}$ values at $25^{\circ} \mathrm{C}$ [reprinted with permission from ref. 236 . Copyright 2016 , American Chemical Society]. 


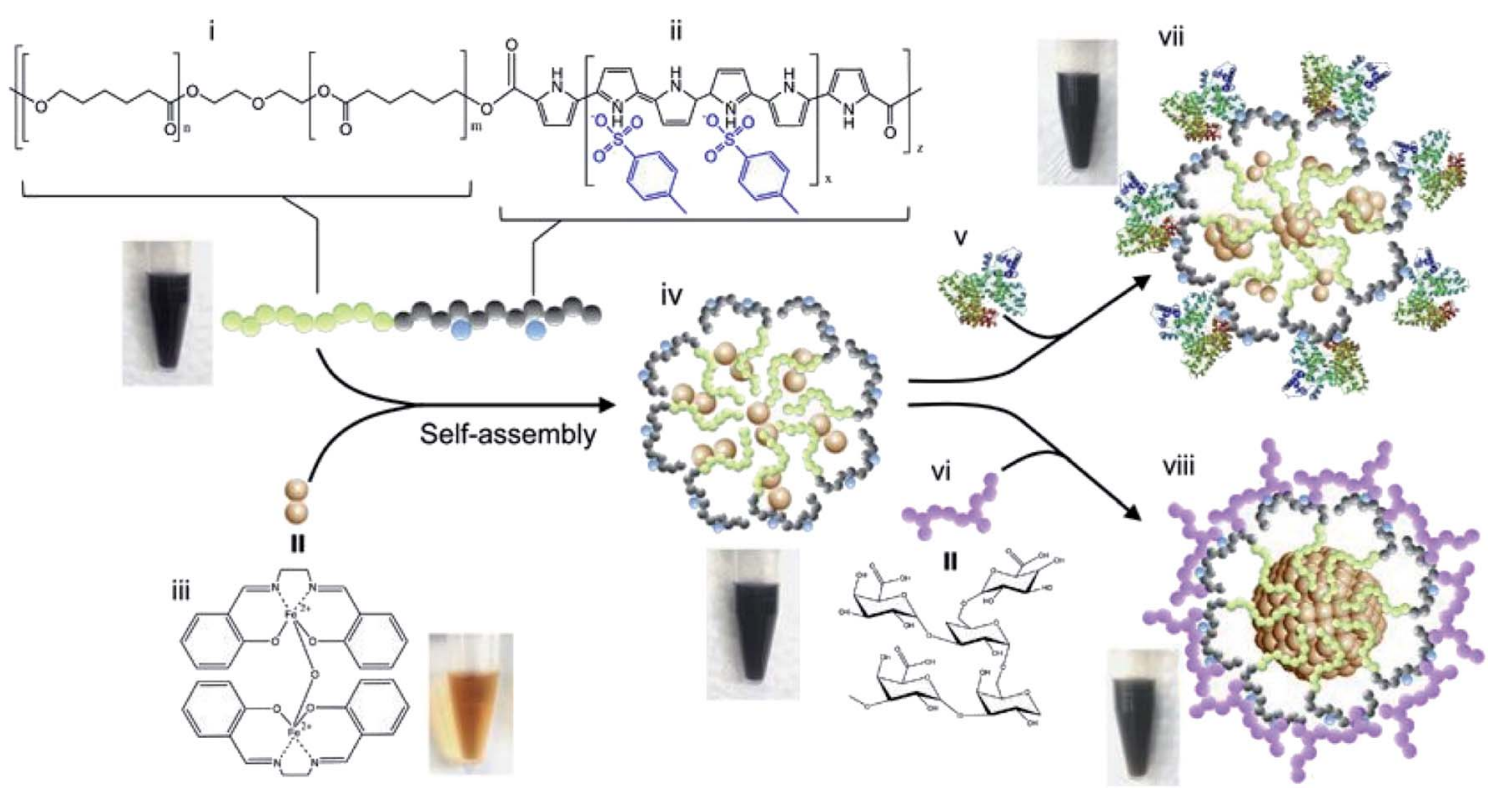

Fig. 28 Magnetic stimuli-mediated precision therapeutics. Preparation of Fe(salen)-loaded polypyrrole (PPy)-PCL core-shell nanoassembled composites (iv) and core size modulation induced by biofunctionalization with bovine serum albumin (BSA) (vii) and gum arabic (GA) (viii). (i) PCL; (ii) PPy (benzosulfonate-doped); (iii) Fe(salen); (iv) Fe(salen)-loaded nanoassemblies; (v) BSA; (vi) GA; (vii) BSA-coated Fe(salen)-loaded nanoassemblies; (viii) GA-coated Fe(salen)-loaded nanoassemblies. Photos of the suspension are displayed at each stage of formulation [reprinted with permission from ref. 237. Copyright 2017, Springer Nature].

(Fig. 20a). ${ }^{223}$ The swollen NCs in acetone were loaded with malachite green as a model payload and transferred to watercontaining DDS (Fig. 20b and d). Oxidation-induced release of malachite green was observed when $\mathrm{FeCl}_{3}$ was added as an oxidizing agent for PFcMA shell segments (Fig. 20c).

A redox-responsive Fc-containing amphiphilic BCP, PEG- $b$ poly(2-(methacryloyloxy)ethyl ferrocene-carboxylate), was used for controlled drug release. ${ }^{224}$ Because of the redox-responsive self-assembly and disassembly property, the vesicles were used to load the RhB model drug for controlled release upon external redox stimuli. In particular, rapid release of $\mathrm{RhB}$ from vesicles was observed during the oxidation by $\mathrm{H}_{2} \mathrm{O}_{2}$ (Fig. 21).

Well-defined redox-responsive Fc-containing amphiphilic BCPs, poly( $N$-acryloylmorpholine)- $b$-poly(2-acryloyloxyethyl ferrocenecarboxylate) $\left(\mathrm{PACMO}_{95}-b-\mathrm{PAEFc}_{25}\right)$, and their selfassembled micelles were used as DDSs. ${ }^{225}$ The copolymer nanomicelles encapsulated model drug paclitaxel (PTX) with EE of $61.4 \%$ (Fig. 22a). These redox-responsive PTX-loaded nanomicelles will enter the cancer tissues through blood circulation and endocytosis. The release rate was controlled by the type and concentration of oxidizing agents, as well as by the release media (Fig. 22b and c).

The water-insoluble model drug Nile Red (NR) was encapsulated in the hydrophobic poly(vinylferrocene) (PVFc) interior of the core-shell nanomicelles based on well-defined PVFc- $b$ PEG diblock copolymers. ${ }^{226}$ Through treatment with $\mathrm{H}_{2} \mathrm{O}_{2}$, instantaneous and quantitative dye release was observed due to oxidation of the PVFc segments (Fig. 23). After the cross-linking treatment, the residence time of the encapsulated NR was significantly increased, and the newly formed shell around the PVFc core was able to keep the encapsulated model compound in it for several months without any significant diffusion from the hydrophobic interior.

Redox-responsive porous multicompartment vesicles were constructed from a linear $\mathrm{ABC}$ triblock terpolymer, poly[2(dimethylamino)ethylmethacrylate]-b-poly(benzyl methacrylate)- $b$-poly(4-vinylbenzylferrocenecarboxylate) (Fig. 24). ${ }^{227}$ These vesicles can be used as smart DDSs to encapsulate and release payloads when the size of the guest is smaller than the pore diameter.

Fc-modified phospholipid (Fc-DSP)-based vesicles are capable of transferring drugs to target cancer cells and selectively trigger their release (Fig. 25a). ${ }^{228}$ Both prepared giant and large unilamellar vesicles (GUVs and LUVs) showed effective loading of model drugs, including the anticancer agent DOX. When exposed to an oxidant solution, external Fc moieties of Fc-DSP vesicles were oxidized to positively charged Fcium groups, which led to the release of the encapsulated drugs. The in vitro experiments with DOX-loaded redox GUVs were performed in adenocarcinoma cervical cancer cells (HeLa) and normal lung fibroblast cells (MRC-5) (Fig. 25b). A significant number of DOX groups were only absorbed by HeLa exposed to redox active GUVs (Fig. 25c). Thus, cancer cells trigger the release of a drug from redox vesicles due to a local redox gradient, namely, the selective redox triggering of liposomes uses their cytotoxic payload at the cancer site.

Of interest are redox-responsive $\mathrm{Pt}(\mathrm{II})$ prodrug micelles based on FA-PEG- $b$-poly( $\alpha$-Pt(II)-SS-caprolactone/caprolactone) copolymers. ${ }^{229}$ They are relatively stable under physiological conditions, but are sensitive to reduction conditions that can trigger the release of conjugated platinum drugs. A dual drug backbone-shattering polymer (DDBSP) based on 
(a)

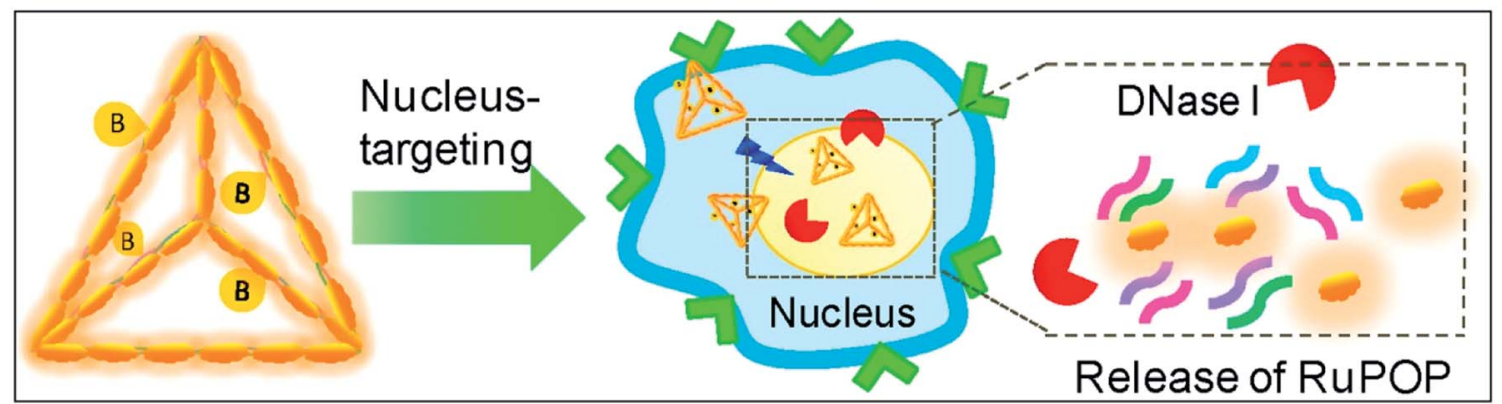

(b)
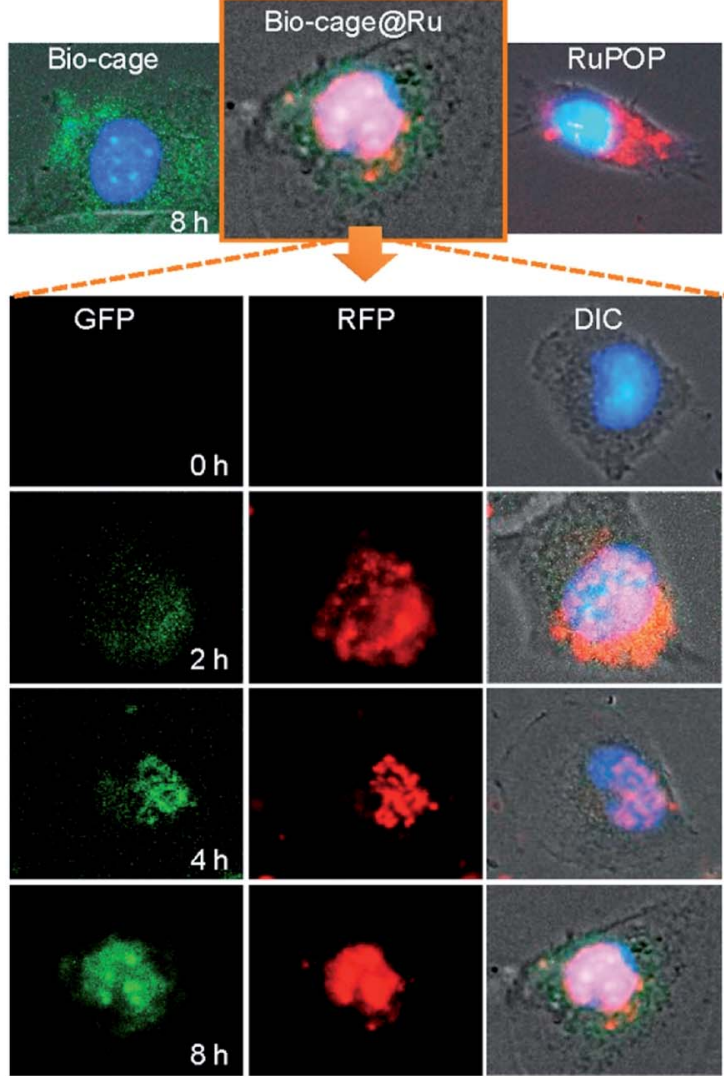

(c)

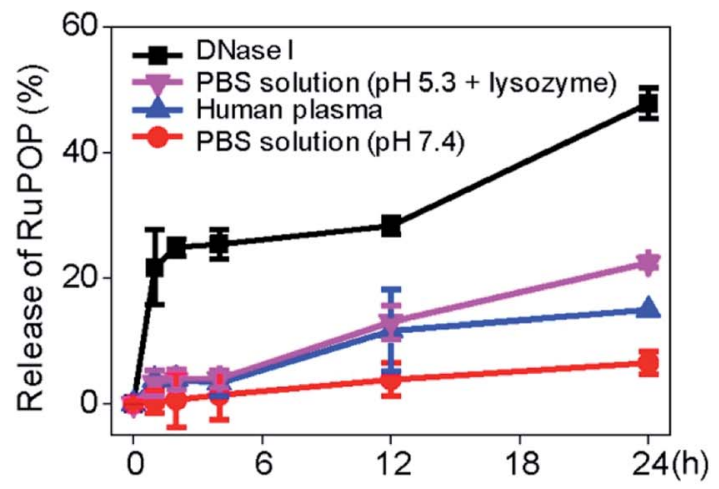

(d)
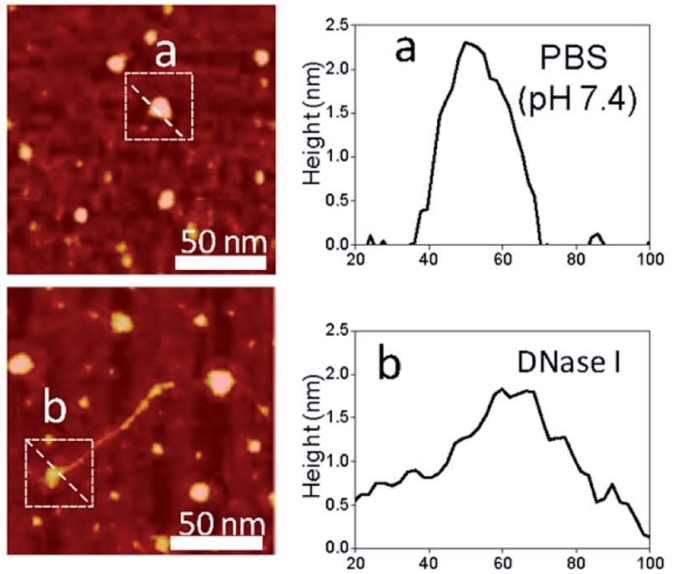

Fig. 29 Translocation and drug release behavior of Bio-cage@Ru in nucleus. (a) Schematic illustration of the subcellular localization and release behavior of Bio-cage@Ru in HepG2 cells. (b) The fluorescence images of the trafficking of Bio-cage@Ru in HepG2 cells. The first line of images recorded the localization of free Bio-cage $\left(20 \mu \mathrm{g} \mathrm{mL}{ }^{-1}\right)$, Bio-cage $(a R u(5 \mu \mathrm{M})$ and free RuPOP $(5 \mu \mathrm{M})$ in HepG2 cells at $8 \mathrm{~h}$ of incubation. The following images detailed the trafficking of Bio-cage $a R u$ in HepG2 cells for $8 \mathrm{~h}$ of incubation. The GFP images recorded the trafficking of Cy3labeled Bio-cage from Bio-cage@Ru. The RFP images represented the fluorescence of RuPOP from Bio-cage@Ru. (c) The release rate of RuPOP from Bio-cage $a R u$ in PBS solution at pH 7.4, human plasma, PBS solution at pH 5.3 supplemented with $1 \mathrm{mg} \mathrm{mL}^{-1}$ lysozymes or DNase I respectively. Values were represented as means \pm SD of triplicate. (d) The AFM images and the size changes of Bio-cageaRu with or without the treatment of DNase I. Scale bar $=50 \mathrm{~nm}$. The size and height of zone a and b were analyzed in histogram a and b respectively [reprinted with permission from ref. 238. Copyright 2016, Elsevier].

demethylcantharidin (DMC) and the Pt(Iv) prodrug was developed. ${ }^{230}$ Under reductive and acidic TME, nano-sized MPs can undergo site-specifically chain-shattered by converting Pt(Iv) to $\mathrm{Pt}(\mathrm{II})$ and hydrolysis of the DMC precursor, releasing both drugs for a controlled synergistic antitumor effect. The size, composition, and morphology of engineered MOFs can be finely tuned to optimize their properties for drug loading and controlled release, demonstrating the potential to serve as promising DDSs. ${ }^{125}$

Even though exciting research is occurring in the area of redox stimuli-responsive polymer nanocarriers, it is difficult to achieve specific redox molecular mechanism-based controllability due to the complex biological environment and heterogeneity of tumor cells. 

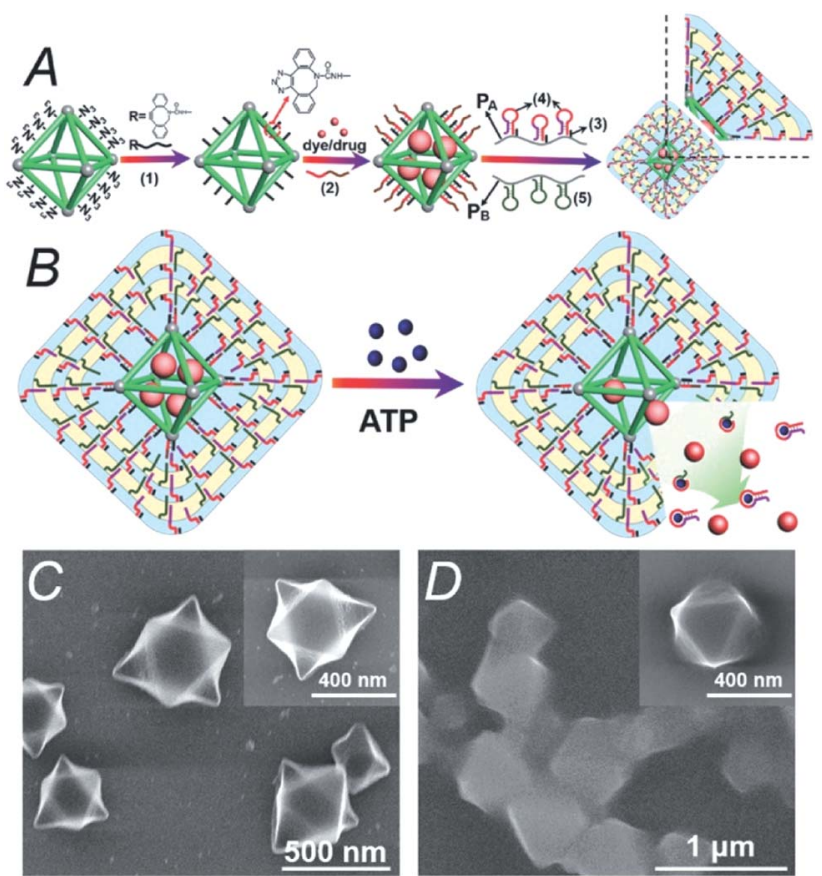

Fig. 30 (A) Synthesis of DOX-loaded MOFs modified with ATPresponsive hydrogel. (B) In the presence of ATP, the decomposition of the modified hydrogel accelerates the release of DOX from MOFs. (C) Scanning electron microscopy (SEM) image of the MOFs. (D) SEM image of ATP-responsive hydrogel-modified MOFs [reprinted from ref. 239 with permission from The Royal Society of Chemistry].

\section{3 pH-responsive DDSs}

Among a variety of environmental triggers, $\mathrm{pH}$ gradients are commonly used to create intelligent responsive DDSs. For example, a class of smart MPs based on platinum prodrug conjugated PAMAM dendrimers with the supersensitive size switching effect in response to $\mathrm{pH}$ changes in the TME was developed to increase penetration into the tumor and efficient drug delivery in vivo. ${ }^{231}$ In another study, a responsive nanocarrier (BLZ-945SCNs/Pt) was obtained, which is able to spatially target both tumor-associated macrophages and tumor cells for chemo-immunotherapy. At pH 6.7-6.8, ionization of the amine groups on the amphiphilic polymer leads to structure collapse of the carrier in the pre-vascular regions of the tumor tissues, which allows the simultaneous release of both platinum prodrug nanoconjugates and small molecule inhibitor BLZ-945 of colony stimulating factor 1 receptor (CSF-1R) of tumorassociated macrophages (TAMs). It turned out that the customized pH-sensitive co-delivery nanocarriers not only induce tumor cell apoptosis, but also modulate the tumor immune environment and eventually augmented the antitumor effect of $\mathrm{CD}^{+}{ }^{+}$cytotoxic $\mathrm{T}$ cells due to TAM depletion. ${ }^{232}$

A method was developed for the manufacture of tastemasked oral DDS based on CP, Fe-4, $4^{\prime}$-bipyridine (Fe-bipy) complex, that regulated the release of unpleasant drug taste by changing the $\mathrm{pH}$ value in the physiological environment of the alimentary canal (Fig. 26). ${ }^{233}$ The pH sensitive Fe-bipy was grafted onto MSNPs containing the model bitter drug mequindox (MEQ) in their mesopores. In artificial saliva $(\mathrm{pH}$ 6.6), Fe-bipy CPs effectively prevent the leakage of a loaded guest molecule MEQ. On the other hand, in the artificial gastric fluid ( $\mathrm{pH}$ 1.0), the coordination bonds of the Fe-bipy complex were broken, resulting in the release of MEQ molecules from DDS.

Epigallocatechin-3-gallatea palmitate was encapsulated in ZIF-8 NPs with functionalization of FA, which is commonly used as pH-responsive DDS. ${ }^{234}$ By recognizing targets between FA on the surface of NPs and the overexpressed FA receptor (FR) in cancer cells, NPs can efficiently internalize into cells and present targeted effects of inhibition growth on HeLa cells (cancer cells) compared to HEK 293 cells (normal cells), which corresponds to the regulation of the reactive oxygen species (ROS) level and the induction of autophagy. Autophagy flux and autophagosome formation are markedly induced by treating cells by NPs, indicating that $\mathrm{pH}$-responsive NPs with targeted identification for cancer cells can be used as highly effective DDSs for targeting cancer chemotherapy. DOX-loaded ZIF-8 can realize effective $\mathrm{pH}$-responsive release in the cancer treatment, which demonstrates the potential for the production of stimuliresponsive DDSs for widespread use. ${ }^{235}$

It should be noted the use of smart hydrogel G1c.WP6 obtained by forming inclusion complexes between pillar[6]arene (WP6) and Fc groups of polymer network G1c (Fig. 27). ${ }^{236}$ Using $\mathrm{DOX} \cdot \mathrm{HCl}$ as a model drug, drug loading efficiency and $\mathrm{pH}-$ responsive release behavior of the smart hydrogel were confirmed. In vitro experiments demonstrated that rapid release of DOX.HCl is achieved in an acidic media (e.g., $\mathrm{pH} 2.0$ ). Given the acidic microenvironment of tumor cells, the release of DOX $\mathrm{HCl}$ from G1c.WP6 hydrogel can also be triggered in tumor tissues.

Despite the advantages of pH-responsive polymer nanocarriers such as controlled drug release, high specificity to the tumor, intracellular drug delivery, and excellent therapeutic efficacy with reduced side effects, $\mathrm{pH}$-responsive polymer nanocarriers face severe challenges. Although many studies on pH-responsive polymer nanocarriers are still in preliminary stages, in vitro drug release behavior and cytotoxicity data are available. These systems are slow in clinical trials because of defects like polymer-related toxicity and low conjugate bioactivity. In the future, more efforts are needed to develop methods for combinations with other stimuli like redox or temperature for specific targeted release.

\subsection{Magnetic stimuli-responsive DDSs}

Magnetism is an external non-invasive activation method that has attractive abilities, since magnetic fields rarely interact with the patient's body compared to other traditional stimuli, such as $\mathrm{pH}$ and light. Various strategies based on magneticallyresponsive MPs have been developed at present, mainly because of their ability to achieve magnetically-guided targeting and hyperthermia-induced drug release in an alternating magnetic field. As a typical example, we note a magnetically active metallopolymer-based DDS loaded with simple magnetic metal complexes for the effective cancer treatment (Fig. 28). ${ }^{237}$ 
a)
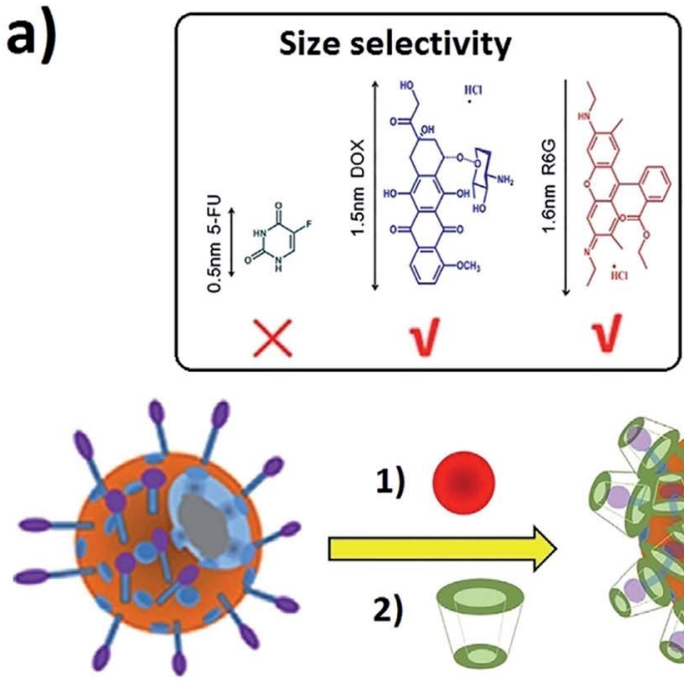

1)
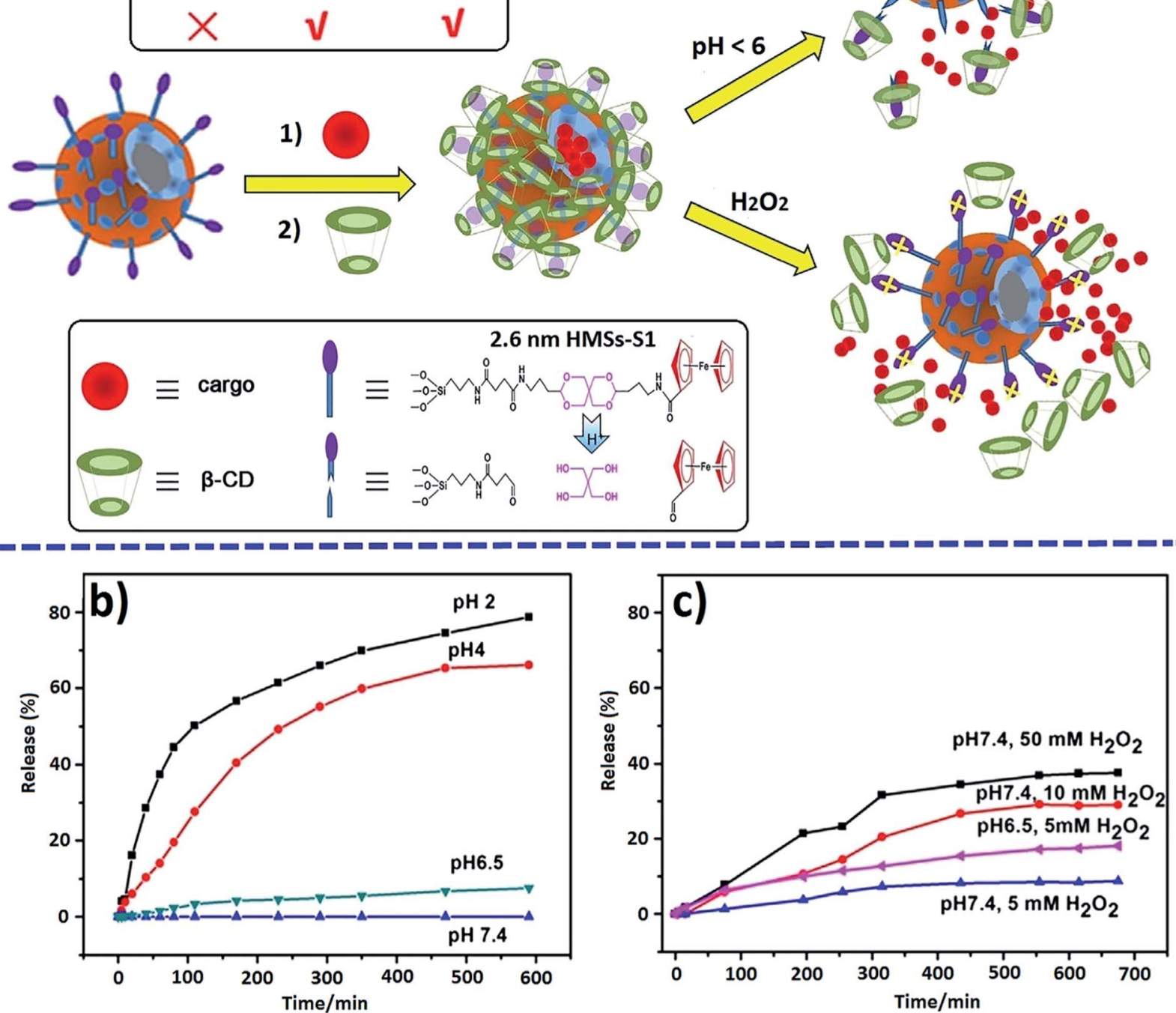

Fig. 31 Schematic diagram of drug loading and release for mechanized HMSs (a), and pH- (b) and oxidation-triggered (c) release profiles of encapsulated R6G [reprinted with permission from ref. 240. Copyright 2016, Elsevier].

The magnetothermal properties triggered the release of the encapsulated dual drug (Fe(salen) and DOX) for accurately deliver the drug to achieve improved in vivo tumor treatment, demonstrating its potential as DDS.

\subsection{Enzyme-responsive DDSs}

Enzyme-responsive DDSs have emerged as a promising option for the accurate delivery of drugs. The use of enzymes as triggers may be useful for the development of therapeutic agents because of their ability to stimulate chemical reactions under physiological conditions. In the past few years, a number of MPs has been used to create enzyme-responsive DDSs. For example, a biocompatible nanostructure of DNA origami was created to deliver the antitumor complex of ruthenium. ${ }^{238}$ The unique tetrahedral DNA nanocages facilitate intercalation with the $\mathrm{Ru}$ (II) complex, increasing the loading efficiency of drugs. Further conjugation with biotin provides specific cellular uptake of the DNA nanocarrier by HepG2 cells by receptormediated targeting. In addition, unlike the free $\mathrm{Ru}(\mathrm{II})$ complex or only nanocage, this system can translocate to the cell nucleus upon internalization and is degraded in response to DNases, which leads to the controlled release of the drug and 
a)
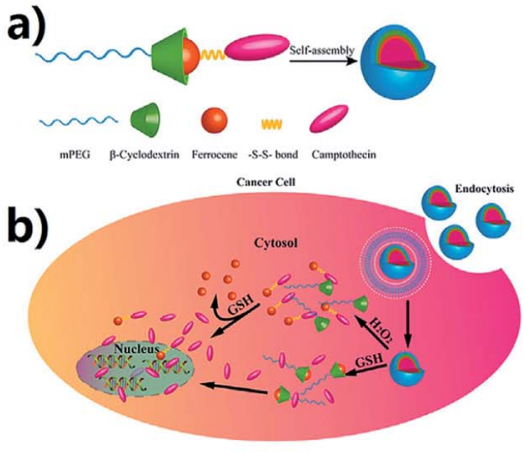
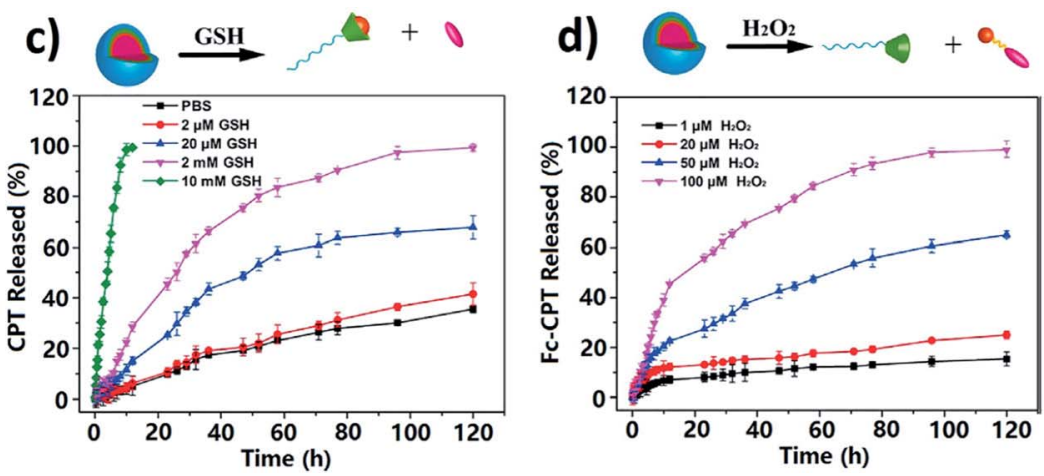

Fig. 32 Schematic formation of mPEG- $\beta-C D / F c-C P T$ supramolecular complex micelles (a) and their $\mathrm{GSH} / \mathrm{H}_{2} \mathrm{O}_{2}$ responsive drug release in cancer cells (b). Release profiles of CPT from the mPEG- $\beta$-CD/Fc-CPT supramolecular complex micelles at different GSH concentrations (c) and $\mathrm{H}_{2} \mathrm{O}_{2}$ concentrations (d) [reprinted with permission from ref. 241. Copyright 2017, American Chemical Society].
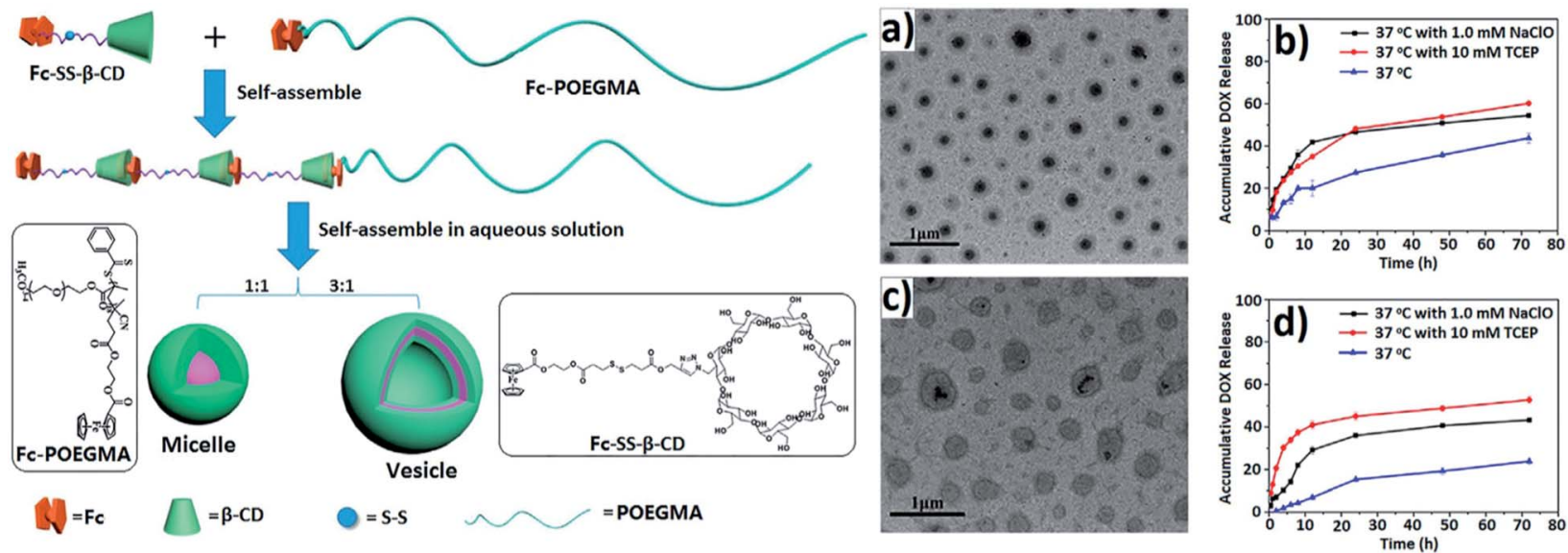

Fig. 33 Schematic formation of micelles and vesicles formed by supramolecular self-assembly of Fc-SS-b-CD/Fc-POEGMA. TEM images of micelles (a) and vesicles (c); in vitro release profiles of DOX from micelles (b) and vesicles (d) [reprinted with permission from ref. 242 . Copyright 2016, American Chemical Society].

subsequent induction of effective cell apoptosis via ROSmediated signaling pathways (Fig. 29).

It should be noted DOX-loaded MOFs modified with adenosine triphosphate (ATP)-responsive hydrogel for cancer treatment (Fig. 30). ${ }^{239}$ This ATP-responsive MOFs/hydrogel showed improved anticancer efficacy and provided an interesting strategy for building stimuli-responsive MOFs-based DDSs.

\subsection{Multi-stimuli-responsive DDSs}

As a typical example of multistimuli-responsive DDS, we note the $\mathrm{pH}$ and redox dual-responsive nanovalve with a long stalk on the surface of hollow mesoporous silica NPs (HMSs) designed for size-selective drug delivery. ${ }^{\mathbf{2 4 0}}$ The leakage of loaded rhodamine 6G (R6G) is insignificant in a neutral medium, which confirms the excellent sealing effect of the developed nanovalve (Fig. 31). In an acidic solution, the $\beta-\mathrm{CD} / \mathrm{Fc}$ supramolecular complexes are removed from the surface of HMSs, which leads to the opening of the nanovalves and the rapid release of $\mathrm{R} 6 \mathrm{G}$ molecules from the pores of HMSs. It is important that the resulting long stalk are effective for loading larger-sized cargos, such as DOX and R6G, but are not suitable for encapsulating small sized 5-FU.

ROS and glutathione (GSH) dual redox-responsive supramolecular micelles were developed based on Fc-CPT and MPEG$\beta$-CD (Fig. 32). ${ }^{241}$ These DDSs exhibit remarkable superfast release of CPT from micelles at high GSH concentration, and controlled release was observed by adjusting ROS (such as $\mathrm{H}_{2} \mathrm{O}_{2}$ ) concentration. In human cancer cells, the amount of ROS is higher than in normal cells, which can oxidize $\mathrm{Fc}$ to $\mathrm{Fc}^{+}$and thus lead to the release of loaded drugs.

Well-defined dual-redox responsive supramolecular micelles and vesicles were developed, based on a supramolecular amphiphilic BCP containing $\beta-\mathrm{CD} / \mathrm{Fc}$ host-guest joints and a traditional hydrophilic homopolymer poly(oligo(ethylene glycol) monomethyl ether methacrylate) with Fc terminus (FcPOEGMA) (Fig. 33). ${ }^{242}$ DOX and DOX $\mathrm{HCl}$ as model drugs were successfully encapsulated in the micelles and vesicles, respectively. Both tris(2-carboxyethyl)phosphine (TCEP) as a reducing agent and $\mathrm{NaClO}$ as an oxidizing agent triggered structural 

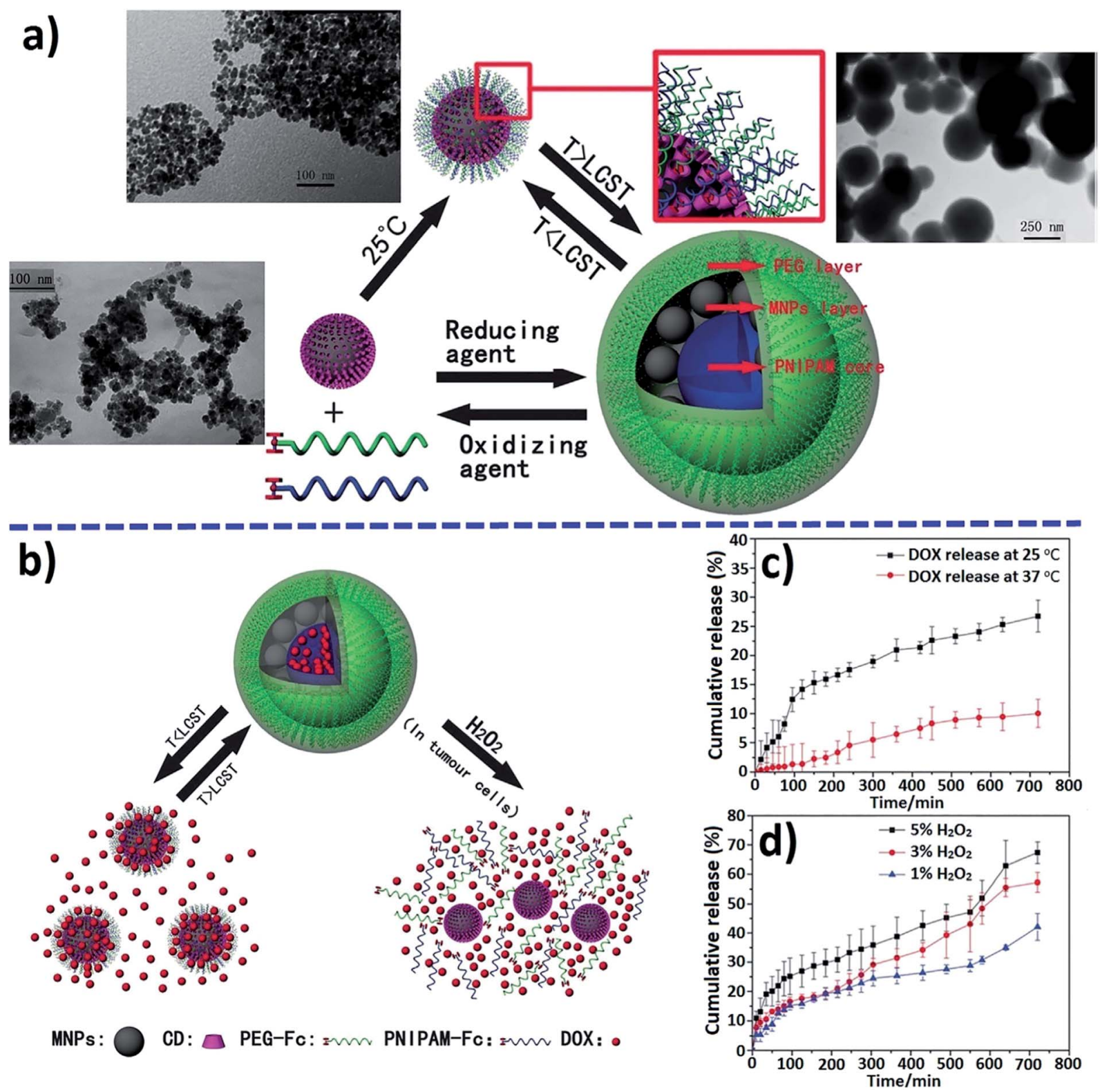

Fig. 34 Illustration of the construction (a) of PEG/PNIPAM/CD-MNP micelles and a possible thermo- and redox-triggered mechanism for drug release (b). Cumulative release of DOX from micelles triggered by (c) temperature and (d) $\mathrm{H}_{2} \mathrm{O}_{2}$ [reprinted from ref. 243 with permission from The Royal Society of Chemistry].

deformation of the supramolecular micelles and vesicles, contributing to the release of DOX (Fig. 33b and d).

Multi-stimuli-responsive magnetic nanomicelles were constructed by surface attachment of hydrophilic PEG chains and thermally responsive poly( $N$-isopropylacrylamide) (PNIPAM) segments (Fig. 34a). ${ }^{243}$ The PEG/PNIPAM/CD-MNP micelles, where MNP is magnetic nanoparticle, had a high loading capacity for DOX and a high saturation magnetization simultaneously. The tunable release of DOX was achieved in response to temperature, $\mathrm{H}_{2} \mathrm{O}_{2}$ or $\mathrm{pH}$ independently or of the combined effect of multiple stimuli. The lowering of the temperature triggered a partial release of free DOX (Fig. 34c), and other DOX were retained on the surface of single small PEG/PNIPAM/CD-
MNP particles (Fig. 34b). The addition of oxidizing agents such as $\mathrm{H}_{2} \mathrm{O}_{2}$ led to the dissociation of the micelles into CDMNPs, and the encapsulated DOX was consequently released (see Fig. 34b). In addition, a faster release of DOX was observed with increasing $\mathrm{H}_{2} \mathrm{O}_{2}$ concentration (Fig. 34d).

Dual-responsive host-guest based colloidal microcapsules (MCs) were made using $\beta$-CD-modified polystyrene (PS) NPs (PSCD NPs) and Fc-grafted polyethylenimine (PEI-Fc) (Fig. 35). ${ }^{244}$ In the drug-loading experiments, the rhodamine isothiocyanate labeled dextran $\left(M_{\mathrm{w}} 150 \mathrm{kDa}\right)$ as a model was mainly encapsulated in the pores between NPs in the shell of MCs.

Both redox- and temperature-responsive supramolecular micelles were developed as tunable releasing nanocarriers for 

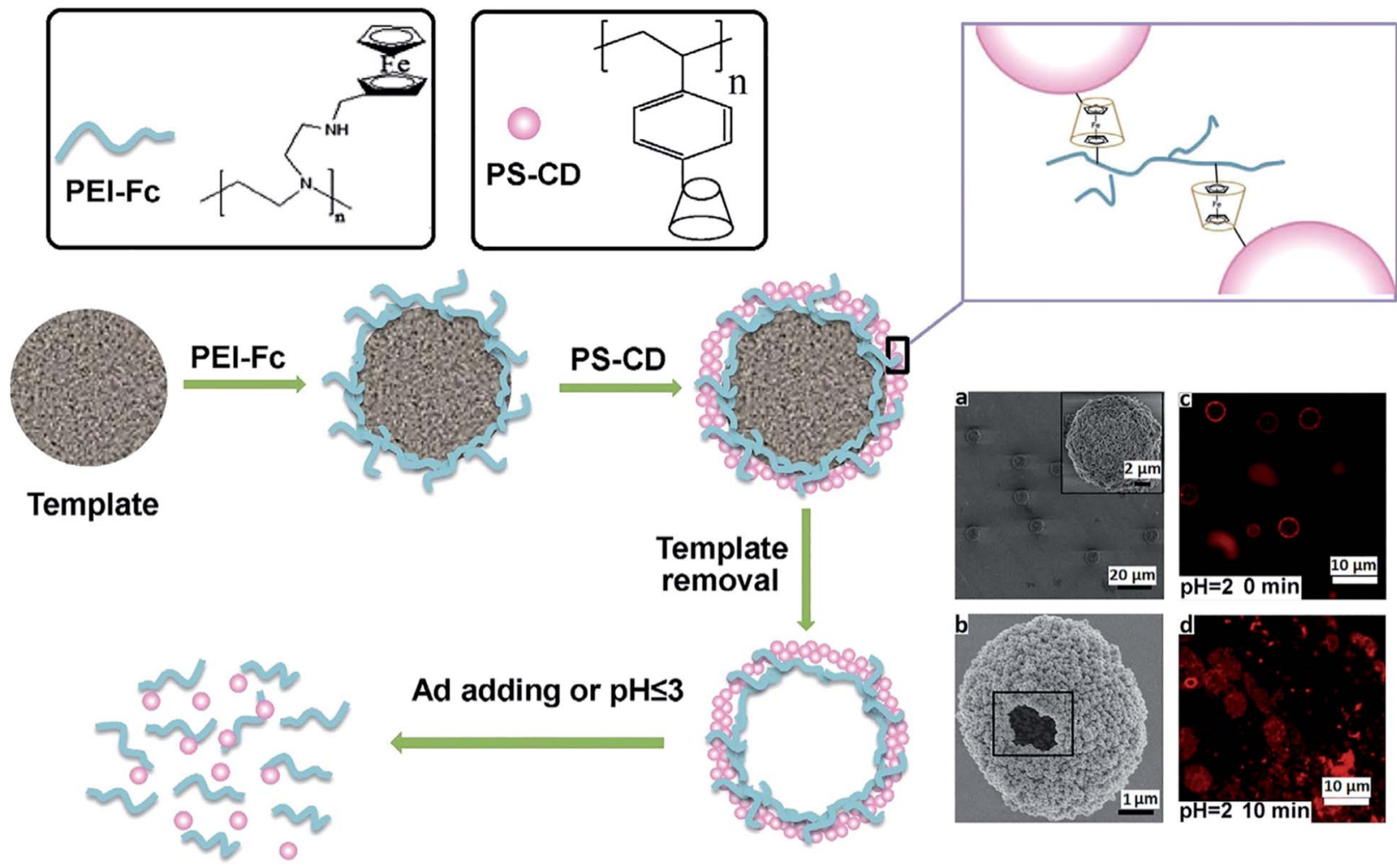

Ad adding or $\mathrm{pH} \leq 3$
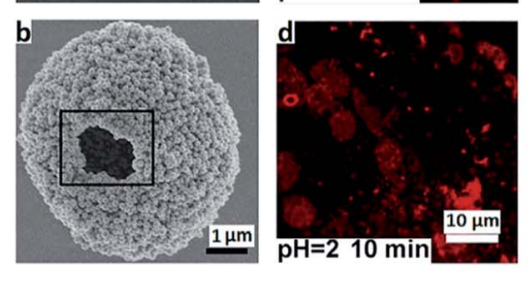

Fig. 35 Schematic illustration of the process of manufacturing and disassembling host-guest based colloidal MCs. (a) SEM image of colloidal MCs before template removal; (b) SEM image of one broken colloidal MC dried by CPD method; confocal laser scanning microscopy images of colloidal MCs at pH 2.0 for 0 (c) and 10 (d) min. Ad is amantadine hydrochloride [reprinted with permission from ref. 244. Copyright 2016, Elsevier].

DDSs (Fig. 36a). ${ }^{245}$ The supramolecular micelles encapsulated hydrophobic drugs, such as DOX, and showed oxidation- and temperature-triggered controllable release behavior. When the ambient temperature was lower than lower critical solution temperature (LCST), for example, $25^{\circ} \mathrm{C}$, a burst of DOX release was observed during the first $15 \mathrm{~h}$, and approximately $91.5 \%$ of DOX was released after $48 \mathrm{~h}$ (Fig. 36d). In the presence of $\mathrm{H}_{2} \mathrm{O}_{2}$, due to the dissociation of $\beta-\mathrm{CD} / \mathrm{Fc}$ inclusion complex, the mPEG-Fc/PNIPAM- $\beta$-CD micelles dissociated into smaller PNIPAM- $\beta$-CD micelles (Fig. 32c), which is accompanied by a partial release of loaded DOX. When the ambient temperature was further reduced to $25{ }^{\circ} \mathrm{C}$ after the redox-triggered release, most of the entrapped DOX in the PNIPAM- $\beta$-CD micelles was released (Fig. 36e) due to the hydrophobic to hydrophilic transition of PNIPAM and the resulting disassembly of hydrophilic polymers.

Thermo- and oxidation-responsive supramolecular vesicles for drug delivery application were constructed by self-assembly of pillar[6]arene $(\mathrm{P}[6]) / \mathrm{Fc}$ based amphiphilic supramolecular diblock copolymers (Fig. 37). ${ }^{246}$ Unique supramolecular vesicles were successfully used to encapsulate an anticancer drug DOX $\mathrm{HCl}$, which was first mixed with the hydrophilic PNIPAM-P [6]-mPEG-Fc at $25{ }^{\circ} \mathrm{C}$, subjected to thermal shock with preheated water, then dialyzed against deionized water at $37{ }^{\circ} \mathrm{C}$ to obtain drug-loaded vesicles. The controlled release of DOX $\mathrm{HCl}$ in the vesicles was achieved by cooling the vesicular solution from $37{ }^{\circ} \mathrm{C}$ to $25{ }^{\circ} \mathrm{C}$, or by exposure to an oxidizing agent such as $\mathrm{AgNO}_{3}, \mathrm{NaClO}$ or $\mathrm{H}_{2} \mathrm{O}_{2}$.

NCs consisting of the triblock terpolymers PVFc- $b$-poly(methyl methacrylate)- $b$-poly $(N, N$-dimethylaminoethylmethacrylate) show triggered release of the payload under three different stimuli, namely, pH change, oxidizing agent, and temperature. ${ }^{247}$ Redox/pH dual-responsive supramolecular vesicles based on the host-guest complexation of Fcium carboxylic acid capped pillar[5]arene (FACP5) and a galactose derivative (G) were used for targeted drug delivery (Fig. 38). ${ }^{248}$ DOX loaded into FACP5G vesicles showed a fast release rate when exposed to GSH in acidic solution ( $\mathrm{pH} 4.0$ ), which makes it possible to control drug release.

Dual stimuli-responsive core-shell microgels based on $\mathrm{P}(\mathrm{NIPAM}-\mathrm{co}$-vinylferrocene) were used to electrochemically switch the polarity (hydrophilicity/hydrophobicity) of the microgel, which provides the electrochemically triggered uptake and release of drugs. ${ }^{249}$ Therefore, bactericidal drugs can be released to effectively kill bacteria. In addition, the good biocompatibility of microgels in cell tests indicates the suitability of the new microgel system as DDSs. A redox- and thermos-responsive gated supramolecular star polymers were obtained using the host-guest complexation of a 6 -fold $\beta$-CD functionalized core molecule and Fc end modified poly $(N, N$ dimethylacrylamide) and poly $(N, N$-diethylacrylamide $)$ linear polymers. $^{250}$ 

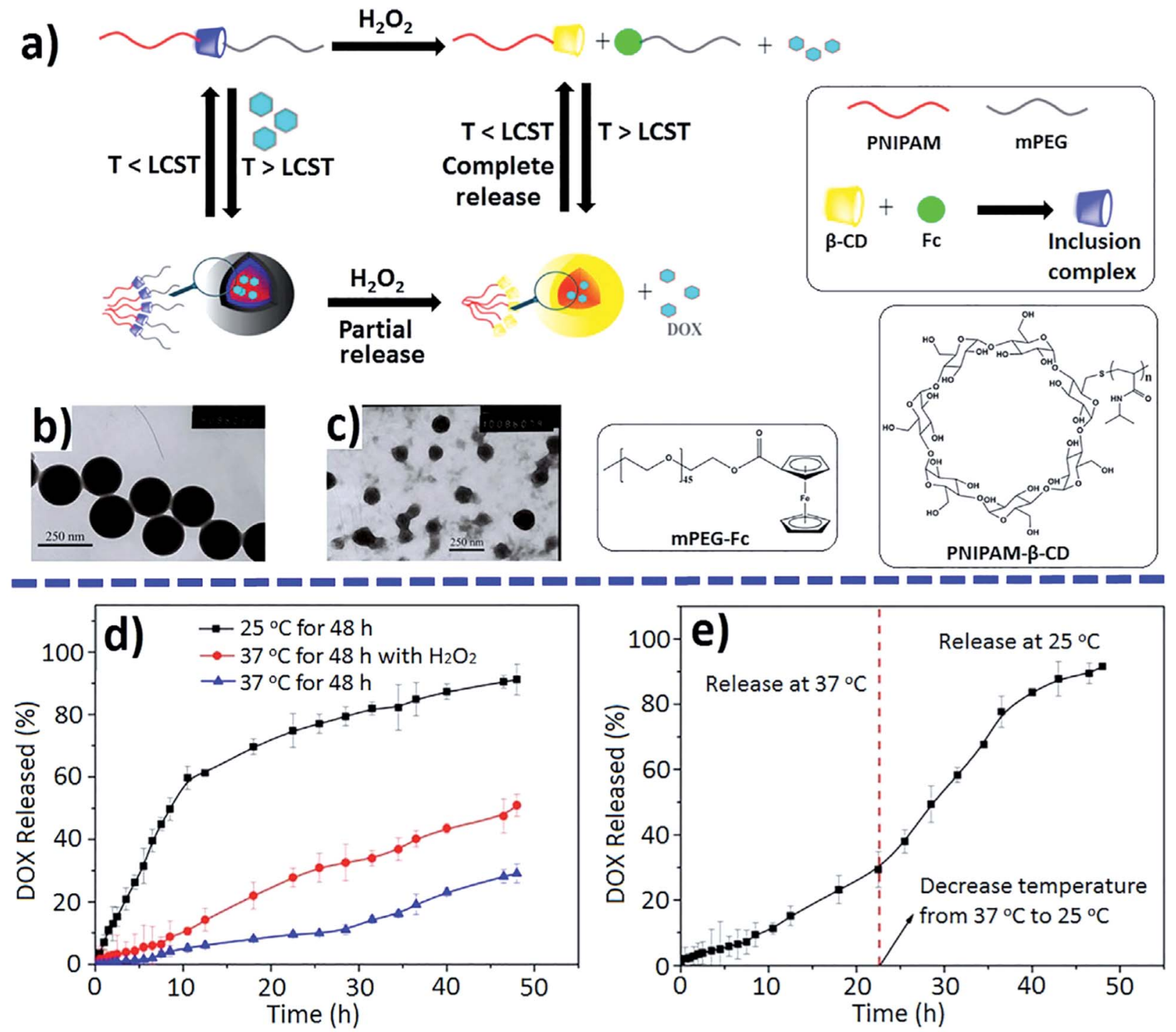

Fig. 36 Schematic dual-stimuli-responsive assembly and disassembly of mPEG-Fc/PNIPAM- $\beta$-CD micelles (a); TEM images before (b) and after oxidation (c) by $\mathrm{H}_{2} \mathrm{O}_{2}$; DOX-released profiles under single (d) or no stimulus and dual stimuli (e) [reprinted with permission from ref. 245. Copyright 2015, American Chemical Society].

In addition to the similar systems discussed here, it should be noted the development of temperature- ${ }^{251-253}$ pressure-,${ }^{254}$ humidity- ${ }^{255}$ competitive binding agent- ${ }^{256,257}$ liposomeresponsive $^{258}$ and other stimuli-responsive metallopolymers used as DDSs. However, using multiple stimuli-responsive polymers is more effective to achieve more precise cancer therapies. Among the most interesting examples, we note $\mathrm{pH} /$ GSH- ${ }^{259,260} \mathrm{NIR} / \mathrm{H}_{2} \mathrm{O}_{2^{-}},{ }^{261}$ and $\mathrm{ATP} / \mathrm{Mg}^{2+}$ ion-responsive systems. ${ }^{262}$

\section{Metallopolymers for combination therapy and multimodal systems}

In this section, we will consider such promising areas for the use of metallopolymer-based DDSs as combination therapy and multimodal systems.

\subsection{Metallopolymers for combination therapy}

Anticancer drug therapy using a single drug is usually ineffective due to the heterogeneity and complexity of cancer and is therefore rarely used in clinical practice. Potential benefits of combination chemotherapy include reduced drug resistance and reduced dosage when equal or higher levels of effectiveness are achieved, thereby increasing its therapeutic index. These DDSs can take many forms to optimize the co-delivery of a wide range of anticancer drugs with different properties. Among the main methods used for combination drug delivery based on polymer carriers, we note polymer-drug conjugates, polymerdrug NPs and polymer-drug conjugates plus polymer-drug NPs (Fig. 39). ${ }^{263}$

A typical example is a polysaccharide-based DDS consisting of Dex decorated with succinic acid (Dex-SA) and loaded with DOX. ${ }^{264}$ The obtained polymer NPs were then cross-linked using 

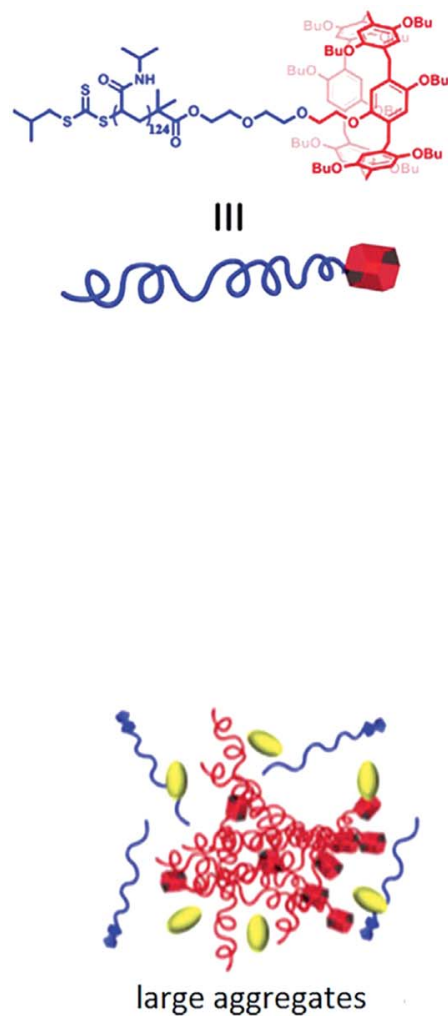

large aggregates

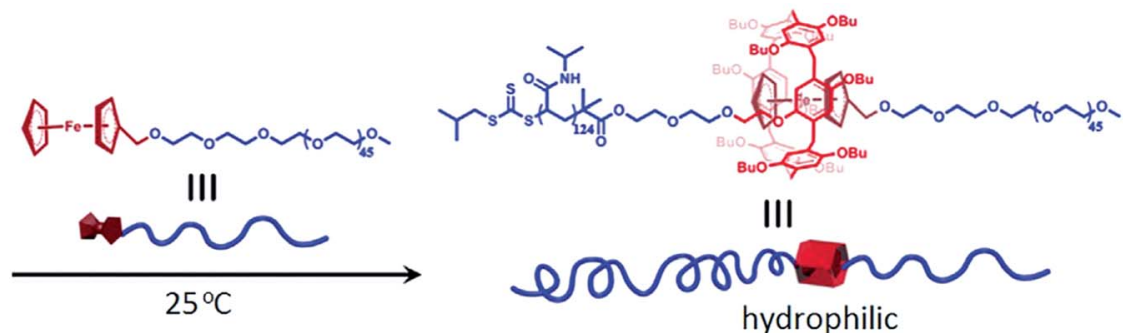

hydrophilic

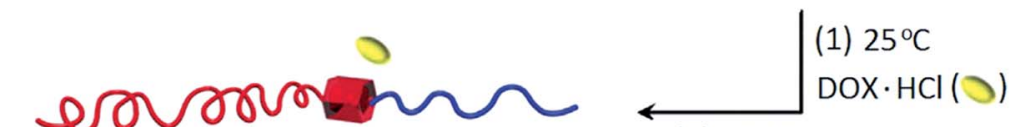

(2) $37^{\circ} \mathrm{C}$
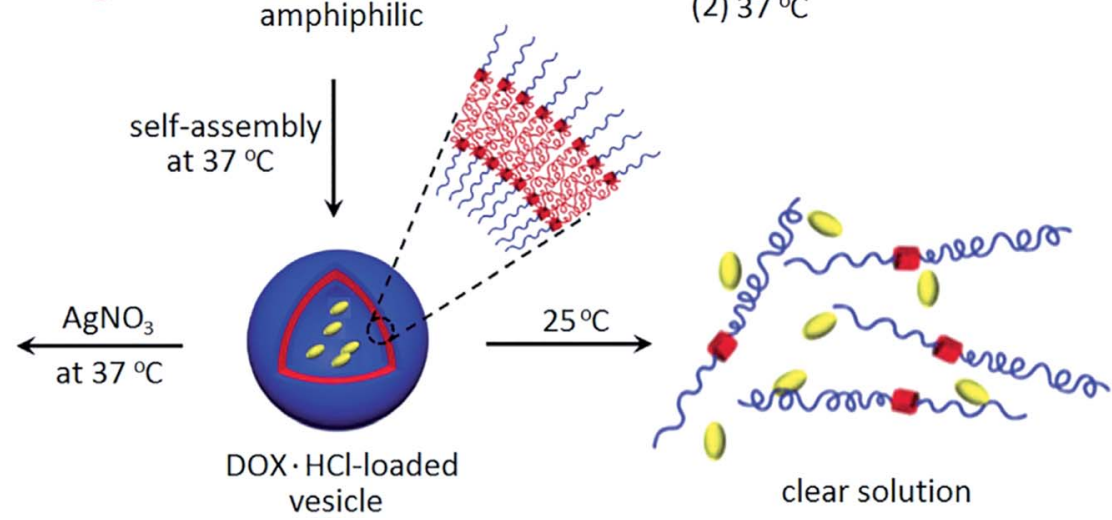

Fig. 37 Illustration of drug loading and thermo- or oxidation-responsive drug release from supramolecular polymer vesicles as a result of selfassembly of PNIPAM-P[6]·mPEG-Fc [reprinted from ref. 246 with permission from The Royal Society of Chemistry].

a cisplatin prodrug to form a combination of DOX-cisplatin particles (Fig. 40). These NPs remained in circulation longer and accumulated in the tumor more significantly than noncross-linked analogues or free DOX. Compared to control groups, the Dex-SA-DOX-cisplatin combination NPs significantly reduced tumor sizes of CT26 colon cancer xenografts. When co-administered with immobilized Arg-Gly-Asp (iRGD), a peptide drug that increases the permeability of tumor tissue, ${ }^{265}$ Dex-SA-DOX-cisplatin therapy successfully suppressed the growth and metastasis of 4T1 murine mammary carcinoma.

Recently, a wide variety of biodegradable polymers has been studied for the co-delivery of several anticancer drugs, including those based on Pt(II) drugs. ${ }^{266-268}$ It should also be noted a grafttype polypeptide micellar system for the combined drug delivery (Fig. 41). This polymer was loaded with cisplatin and docetaxel (DTX), and then was decorated with an $\alpha_{v} \beta_{3}$ integrin targeting peptide $\mathrm{c}(\mathrm{RGDfK})$. In vivo results demonstrated surprisingly long circulation time, minimal side effects, and high antitumor and anti-metastasis efficacy. ${ }^{269}$

Conjugation of the Pt drug, in which the Pt(Iv) pharmacores were coordinated with light-responsive azides as equatorial ligands and with cisplatin-sensitizing norcantharidin as an axial ligand, with biodegradable copolymers led to NPs (Fig. 42). ${ }^{270}$ The mechanism of action of the drug includes damage to nuclear DNA, oxidative damage to DNA and proteins, as well as preventing the repair of damaged DNA. Compared with other types of combination therapy based on the MP systems, this conjugate has several favorable characteristics, including a combination of multiple therapeutic modalities in one conjugate, a high loading capacity of the carrier for each drug with precise control of their stoichiometric ratio, the synergistic effect of all three drugs and controlled drug release triggered by photo-irradiation.

The amphiphilic Dex-Pt(Iv) conjugate was self-assembled in NPs in an aqueous solution and used as a reduction-responsive carrier for DOX encapsulation. ${ }^{271}$ Under reducing conditions, the pendant $\mathrm{Pt}(\mathrm{IV})$ is reduced to $\mathrm{Pt}(\mathrm{II})$, which leads to disintegration of NP and triggered the release of encapsulated DOX. Of interest is the encapsulation of the multifunctional Pt(Iv) prodrug, mitaplatin (Fig. 43a), in PEG- $b$-PLGA micelles. These micelles showed longer blood circulation, lower toxicity, and increased therapeutic efficacy compared with free mitaplatin. ${ }^{272}$ A multifunctional Pt(Iv) prodrug canthaplatin (Fig. 43b) was developed, in which cantharidin (a PP2 A inhibitor that can block DNA repair and inhibit the basic mechanism of platinum drug resistance) was attached to the axial positions of cis-Pt(Iv). Canthaplatin was encapsulated in PEG-b-PLGA micelles, forming NPs capable of combination drug delivery. After the micelles were endocytosed, the multifunctional prodrug was chemically reduced and the cisplatin and PP2 A inhibitors were released in a controlled manner. This combination led to inhibition of DNA repair, which significantly contributed to apoptosis, increased antitumor efficacy and reversed cisplatin resistance to human lung cancer both in vitro and in vivo. 


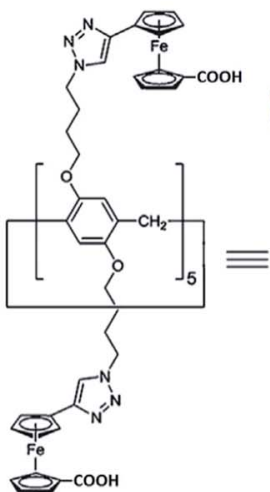

FACP5a
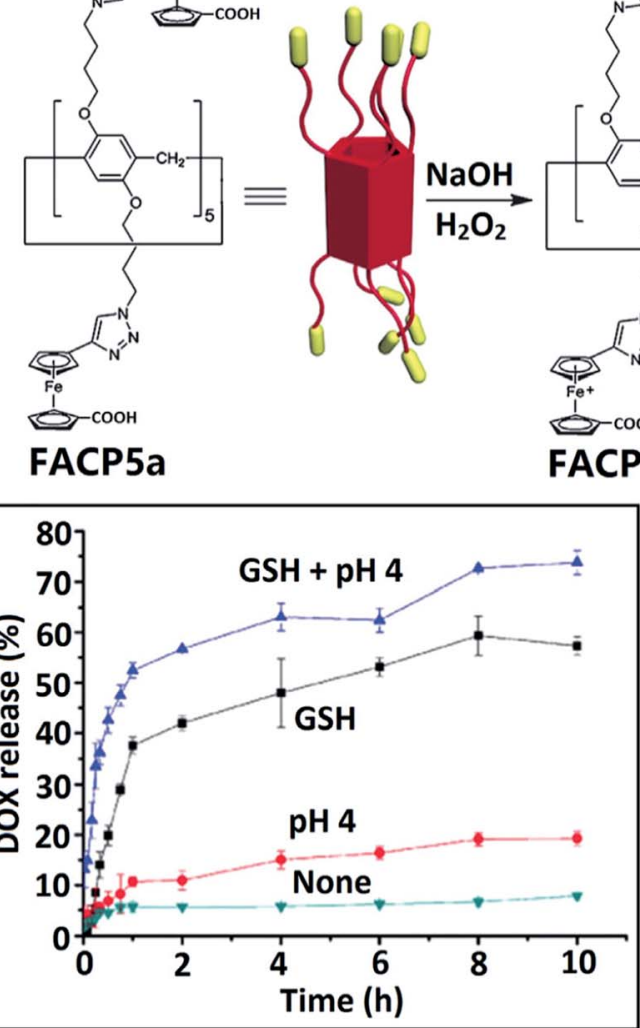

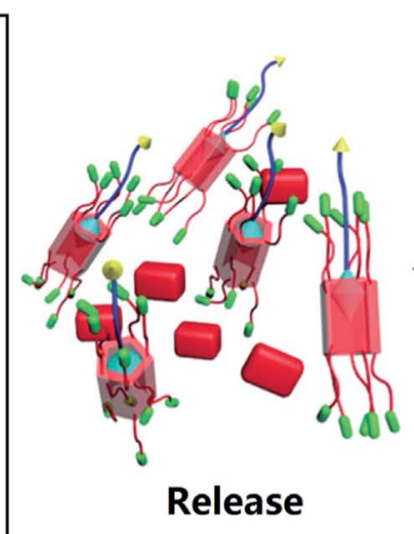

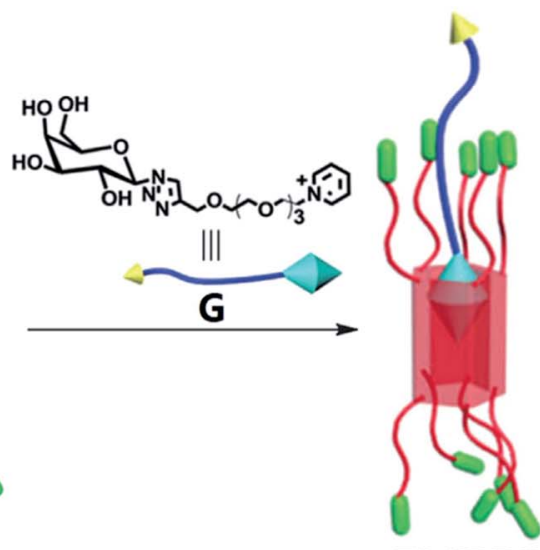

FACP5G

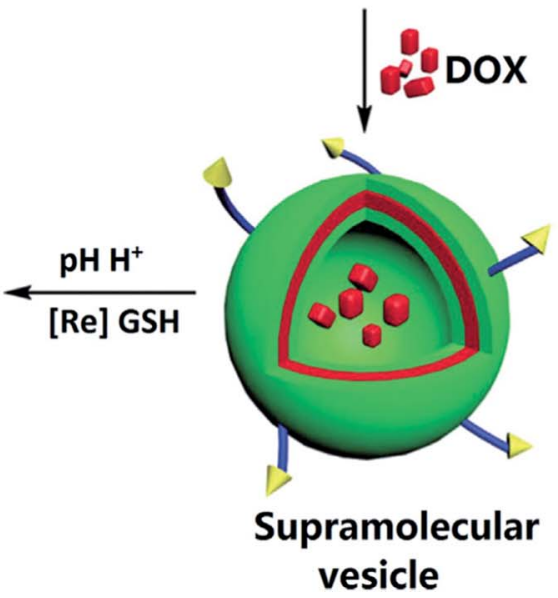

Fig. 38 Illustration of the formation of supramolecular vesicles based on the host-guest complexation of FACP5 and the galactose derivative (G) and their redox/pH dual-responsive drug release. The inset shows the DOX release profiles from DOX-loaded FACP5G vesicles in water [reprinted from ref. 248 with permission from The Royal Society of Chemistry].

(A) Drug conjugate based combination

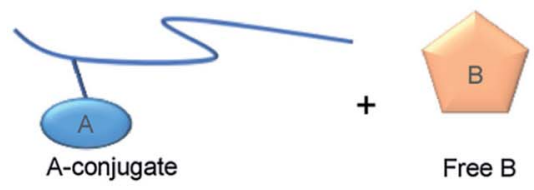

(B) Nanoparticles based combination

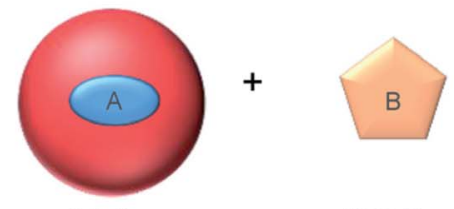

Free B
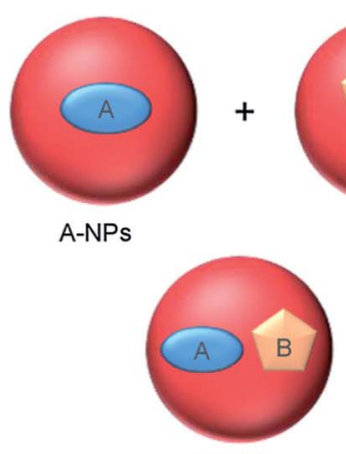

(C) Conjugate + NPs

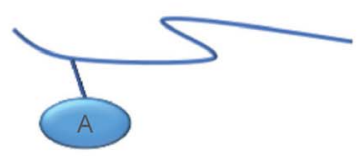

A-conjugate

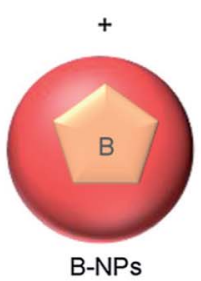

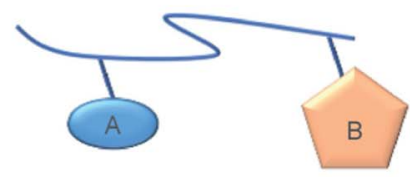

A/B-conjugate

A/B-NPs

Fig. 39 General strategies for combination drug therapy using polymer carriers. (A) The polymer conjugate of drug A can be combined either with the free drug $B$ or with the polymer conjugate of $B$, on the same or different chains, where the "conjugate" refers to any form of the polymer drug that does not form NPs. (B) Drug A can be loaded into polymer NPs, which are then combined with free B or with B NPs, or both A and B can be co-assembled in the same NPs. (C) Drug A can be administered as a polymer conjugate, while drug B is administered in NPs [reprinted with permission from ref. 123. Copyright 2018, Elsevier]. 


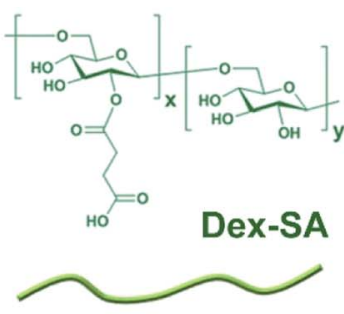

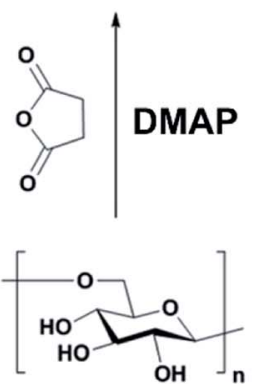

Dextran

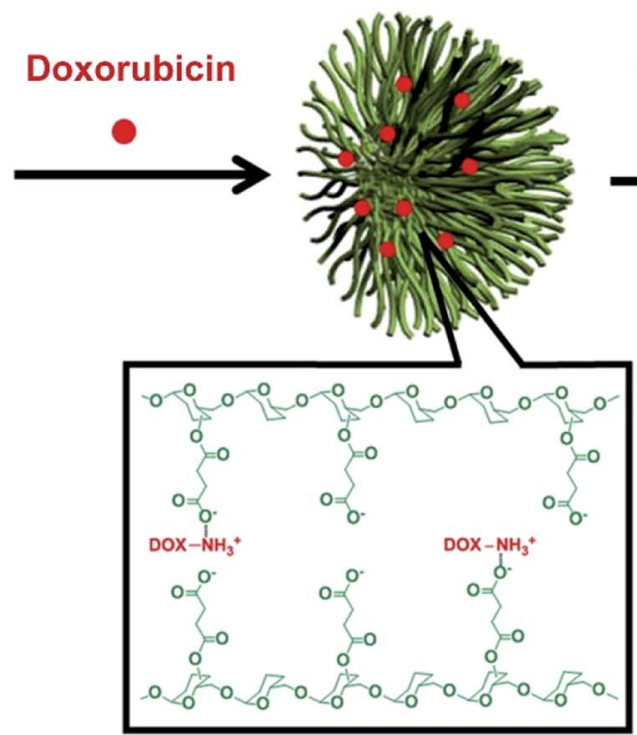

Dex-SA-DOX
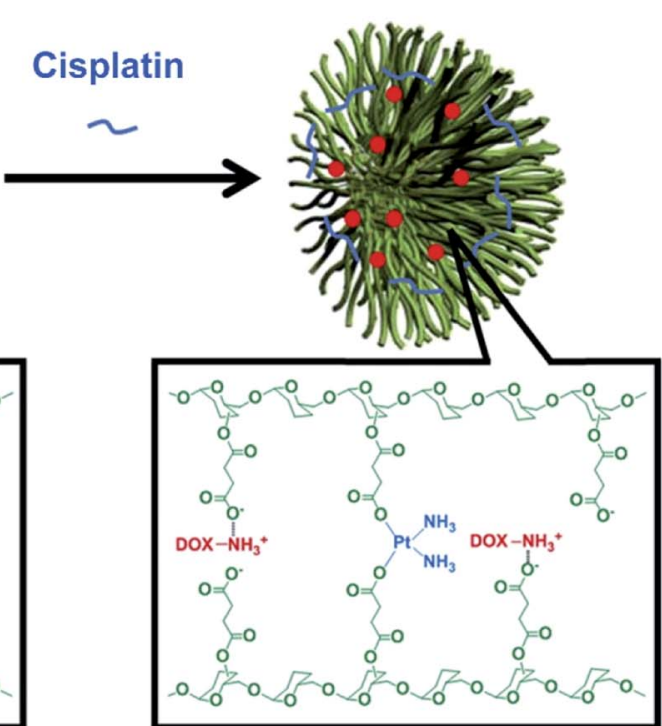

Dex-SA-DOX-CISPLATIN

Fig. 40 The schematic illustration of the process of preparing Dex-SA, Dex-SA-DOX and Dex-SA-DOX-cisplatin [reprinted with permission from ref. 264. Copyright 2014, Elsevier].

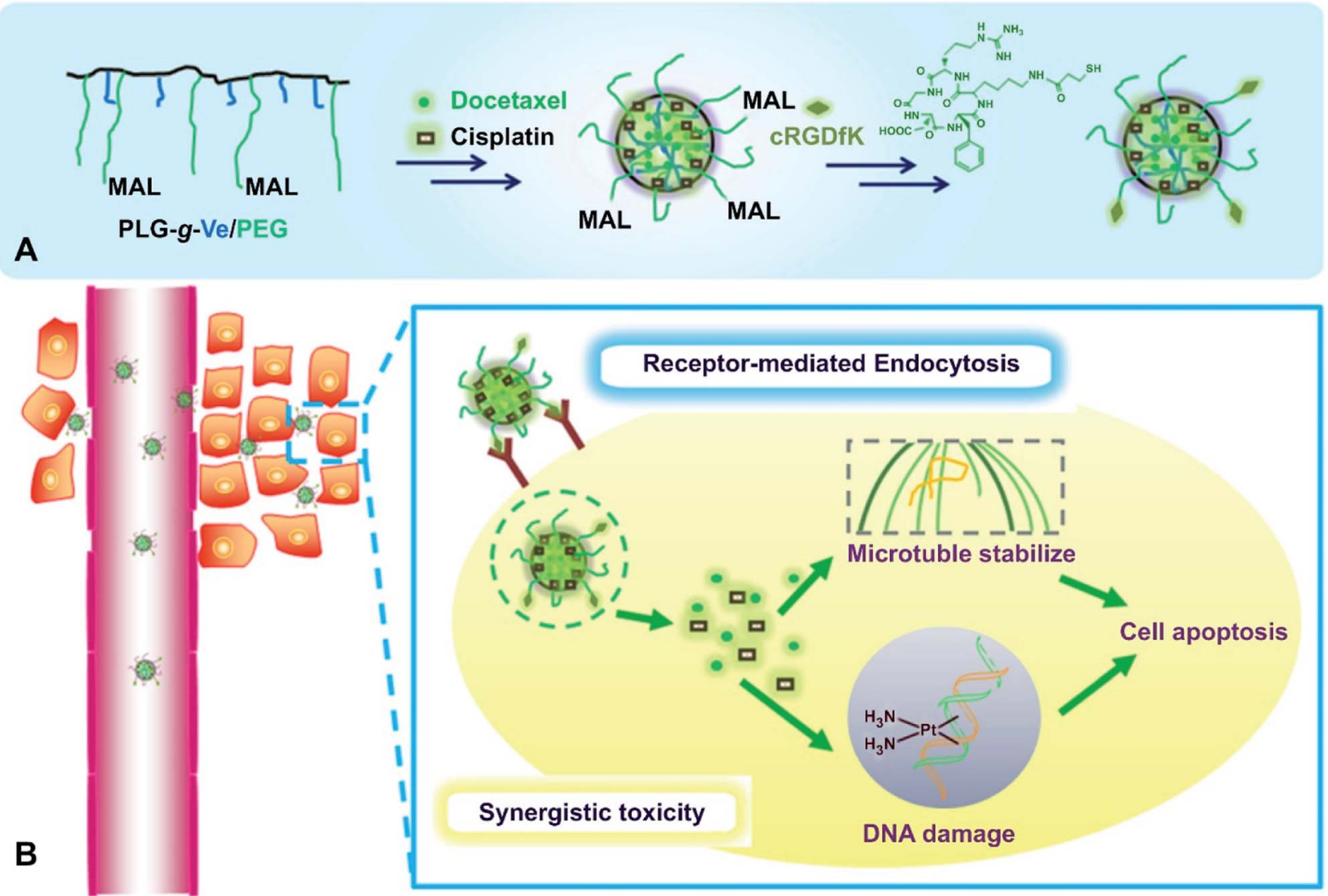

Fig. 41 (A) Schematic illustration of preparation of the c(RGDfK)-decorated dual-drug-loaded micelles. (B) The micelles enter tumor cells by receptor-mediated endocytosis, and the loaded two drugs act synergistically intracellularly [reprinted with permission from ref. 269. Copyright 2014, John Wiley and Sons]. 
a)

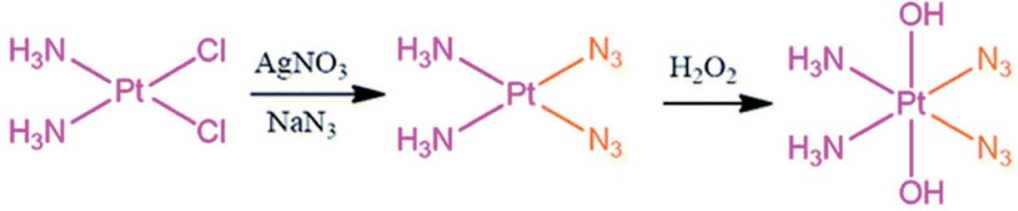

Cisplatin

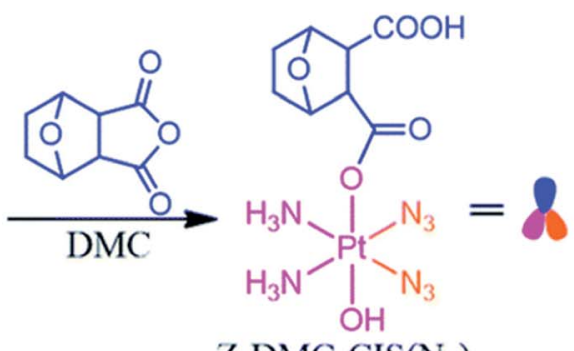

b)
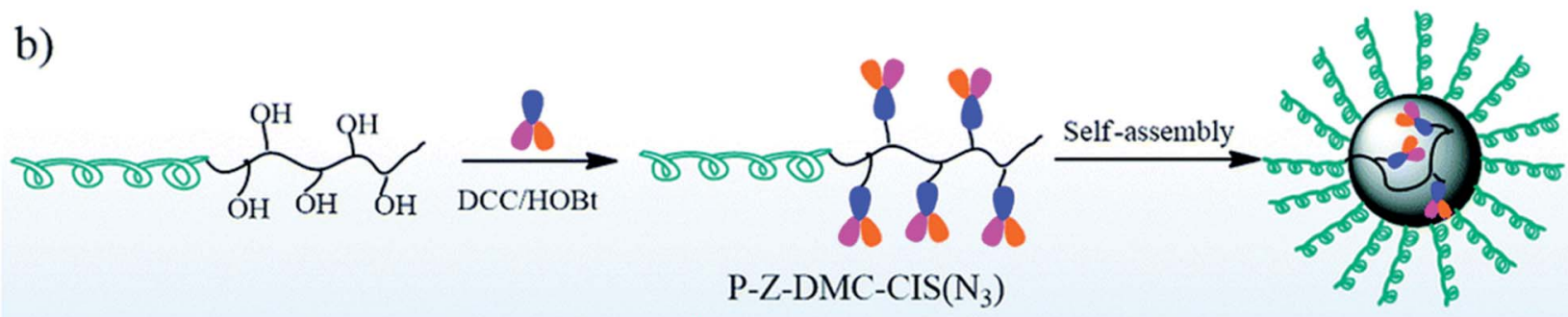

Z-DMC-CIS $\left(\mathrm{N}_{3}\right)$

c)

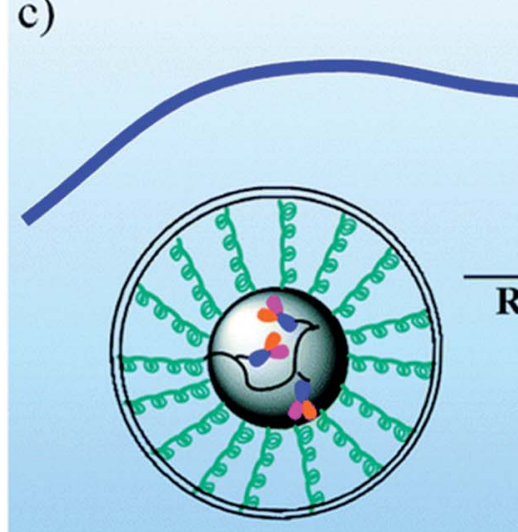

Endosome \& Lysosome

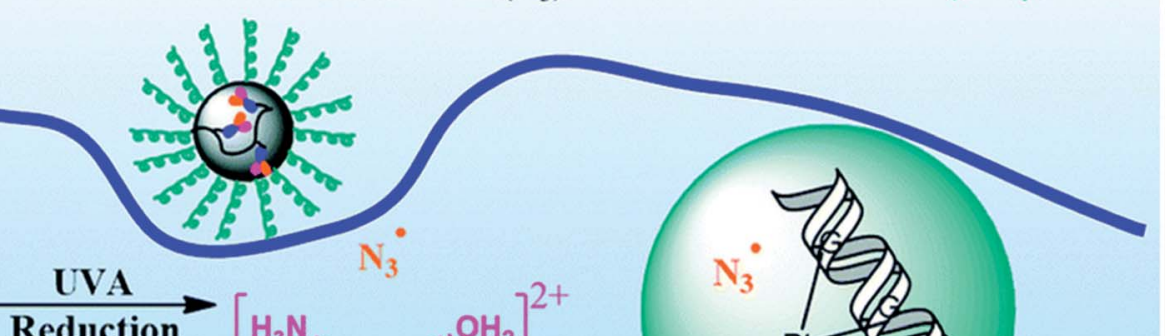

UVA
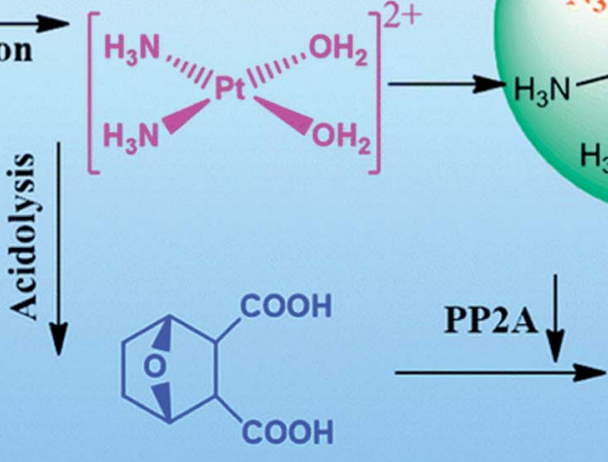

\section{Apoptosis}

Fig. 42 (a) Synthesis of a multifunctional single-drug Z-DMC-CIS $\left(\mathrm{N}_{3}\right)$, (b) conjugation of a single-drug with a biodegradable amphiphilic BCP to obtain a polymer-(multifunctional single-drug) conjugate micelles, and (c) possible paths after the polymer-drug conjugate micelles enter the cisplatin-resistant cancer cells [reprinted from ref. 270 with permission from The Royal Society of Chemistry].

It should be noted that such hybrid DDSs significantly improve the dose-specific delivery of each type of drug, increasing their synergistic efficacy. An example is camplatin, a prodrug formed by coining camphoric anhydride and cisplatin, which was delivered in biodegradable NPs. This system for co-delivery of camphoric acid and cisplatin showed increased anticancer activity compared to cisplatin and successfully overcame cisplatin drug resistance. ${ }^{273}$

A reduction-sensitive DDBSP with dual drugs in its backbone has been developed (Fig. 44A). ${ }^{230}$ Fixed and accurate drug ratios can be released in response to intracellular reduction or acidic conditions, allowing the drugs to be released in a controlled manner. In addition, DDBSP had a high platinum heavy metal content in the polymer backbone, which made it possible to directly monitor the fate of the drug using drug-mediated computer tomography (DMCT). Finally, DDBSP demonstrated (Fig. 44B and C) significantly improved cancer treatment on a high-fidelity patient-derived lung cancer (PDLC).

Of interest is a voltage/pH-driven MSNPs-based multidrug (DOX and GEM) delivery system for multimodal controlled drug release (Fig. 45a). ${ }^{274}$ The unique structure of MSNPs 1 allows two different controlled release methods to be implemented. In particular, when exposed to $+1.5 \mathrm{~V}$ voltage, an accurate controlled release of small GEM is observed due to the selfdissociation of the Fc- $\beta$-CD complex. Raising the ambient $\mathrm{pH}$ to an acidic $\mathrm{pH}$, for example, 6.0, 5.0 and 4.0, results in a gradual release of DOX (Fig. 45b), which was associated with the removal of supramolecular nanovalves caused by the 


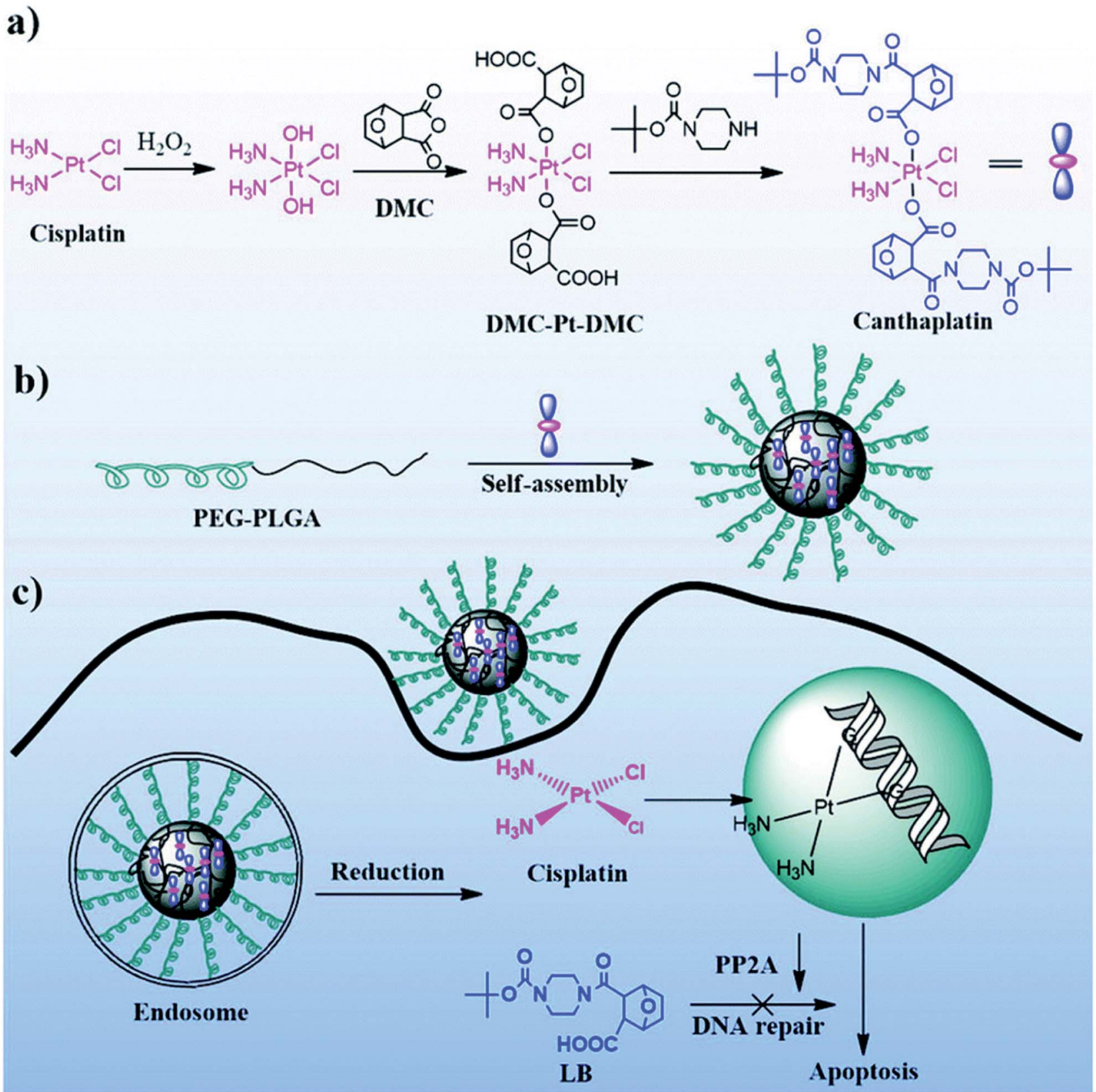

Fig. 43 (a) Synthesis of canthaplatin, (b) preparation of polymer/canthaplatin micelles, and (c) schematic representation of the intracellular action after endocytosis of polymer/canthaplatin micelles [reprinted from ref. 272 with permission from The Royal Society of Chemistry].

splitting of the ketal bond. Being localized in an acidic solution, MSNPs 1 exhibited the concurrent release modality of GEM and DOX (Fig. 45c). Compared to single DOX- or GEM-loaded MSNPs, well-organized MSNPs 1 have improved cytotoxicity to MCF7 cancer cells, indicating potential application in combination therapy to overcome cancer MDR.

The advantages of using metallopolymers-based DDSs in combination therapy include an increase in therapeutic efficacy and a reduction in dosage while achieving equal or higher levels of efficacy and a decrease in drug resistance. In this direction, nanoscale MPs $^{275}$ are of particular attention for combination therapy. ${ }^{276}$ Despite the several advantages these DDSs in combination chemotherapy, many combination treatment regimens that have shown promising in vitro results have been affected by clinical translation because they showed little to no improvement in efficacy and often actually caused higher toxicity. Differences in the physicochemical properties of drugs can lead to dissimilarities in the pharmacokinetics and tissue distribution of each drug.

\subsection{Metallopolymers-based multimodal systems}

Scientists are currently focused on developing multimodal treatment systems to meet the ever-growing demand for effective therapeutic strategies. Over the past few decades, MPs have 


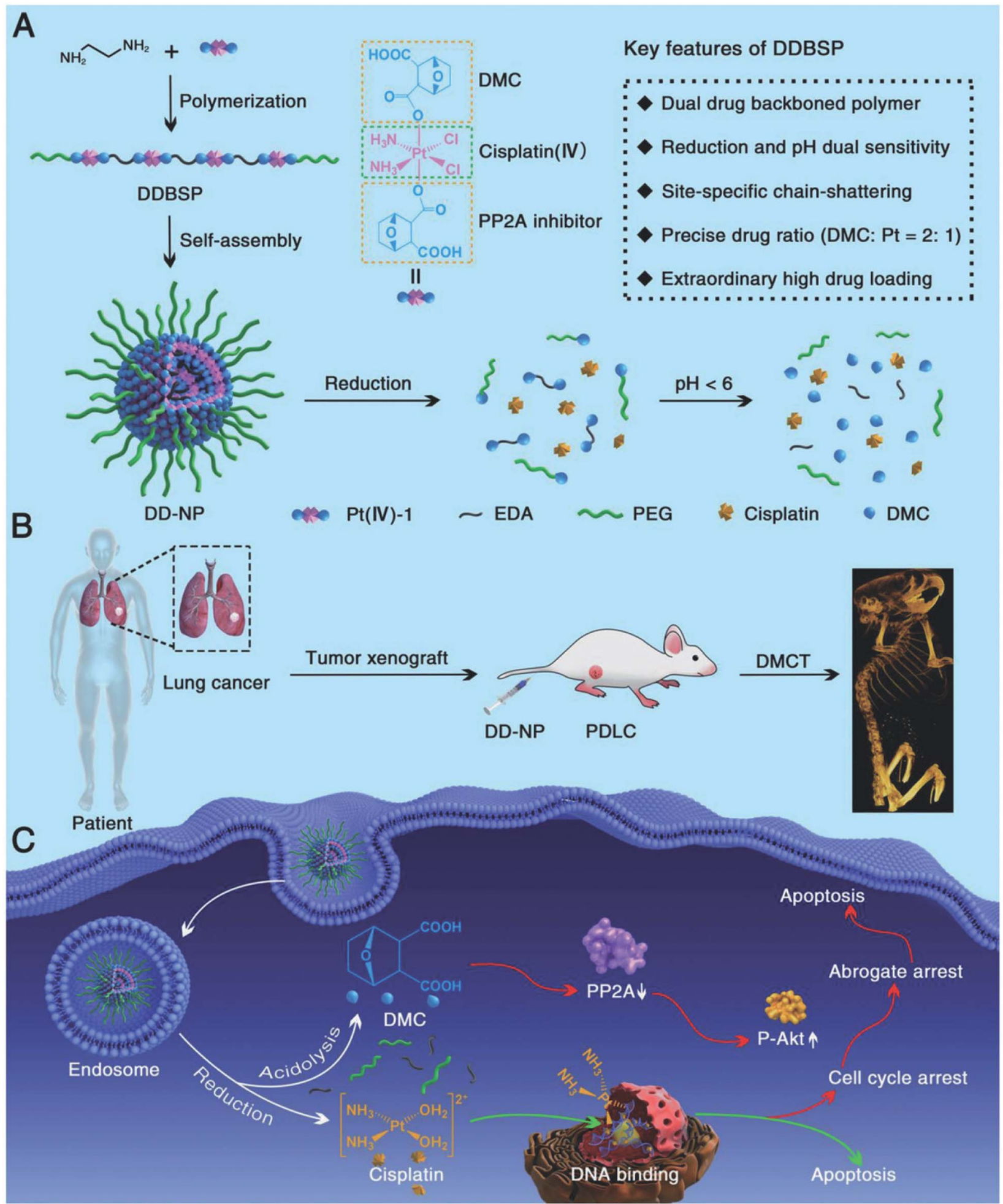

Fig. 44 Schematic illustration of DDBSP for synergistic eradication of PDLC. (A) Illustration of the structure of Pt(IV)-1, the polymerization of DDBSP, self-assembly and chain-shattering of DD-NP with the release of active Pt(॥) and DMC, as well as key features of DDBSP and DD-NP. (B) Illustration of the creation of a PDLC model and Pt-based DMCT after intravenous injection of DD-NP. (C) Possible dual anticancer mechanisms after endocytosis of DD-NP by cancer cells [reprinted with permission from ref. 230. Copyright 2018, John Wiley and Sons].

been synthesized that simultaneously transmit diagnostic information and monitor the in situ therapy process. ${ }^{277}$ The integration of various cancer treatment strategies, such as chemotherapy, photodynamic therapy (PDT) and photothermal therapy (PTT), into the MP nanoplatform has shown great 

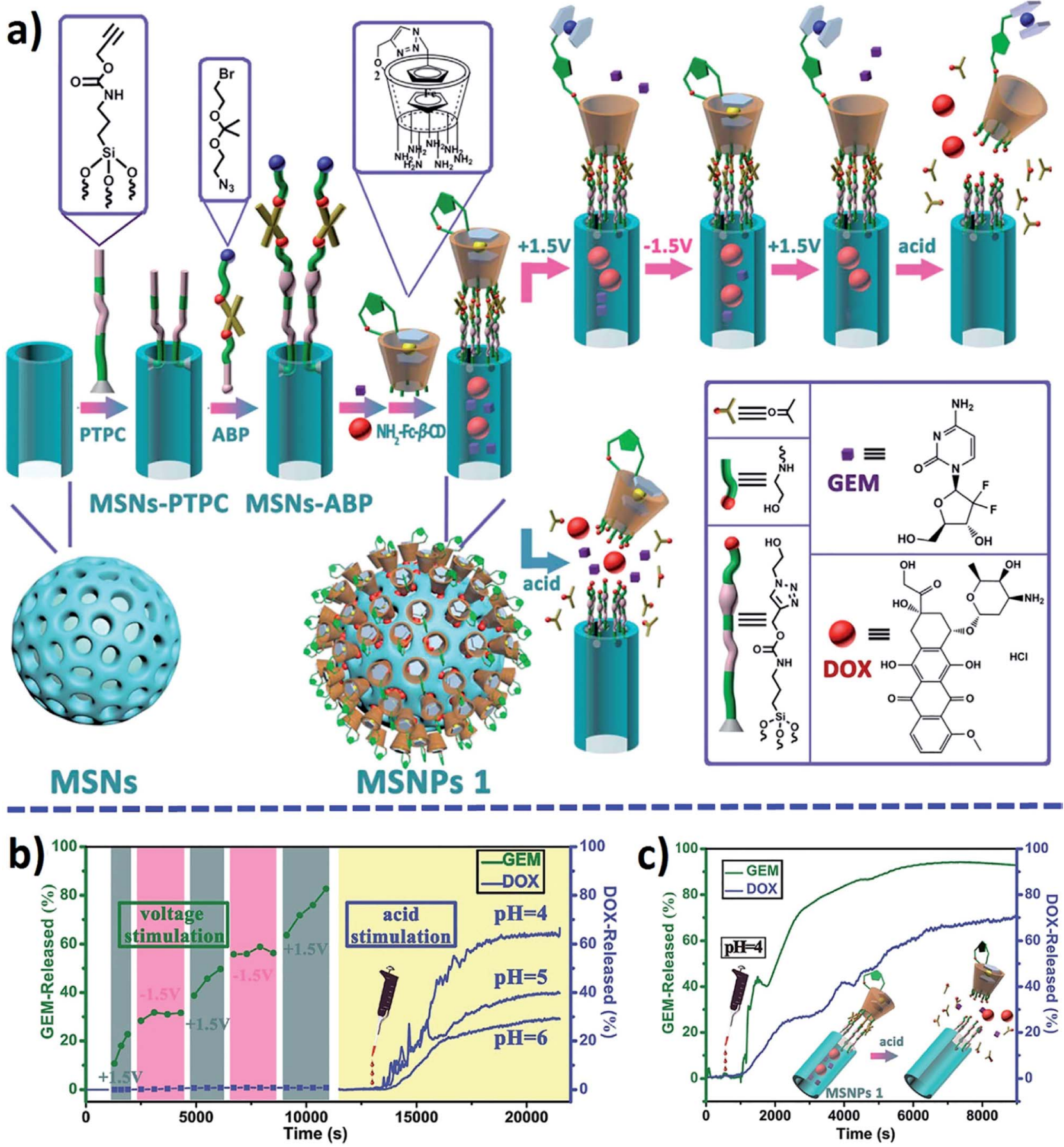

Fig. 45 Schematic assembly and multimodal controlled release modalities of MSNPs 1 (a); sequential (b) and concurrent (c) release profiles of encapsulated GEM and DOX [reprinted with permission from ref. 274. Copyright 2015, American Chemical Society].

potential for obtaining a low risk of recurrence and high anticancer efficacy. ${ }^{278}$

As an example, we note the supramolecular dendritic system, including lipoic acid functionalized dendrons, Pt(II)-PEG conjugate, and NIR fluorophore (dye Cy5.5) for in vivo imaging (Fig. 46). ${ }^{279}$ In vitro studies with lung adenocarcinoma A549 cells showed the internalization of nanostructures due to endocytosis and high cytotoxicity, while in vivo studies demonstrated efficacy comparable to cisplatin with avoiding the nephrotoxicity. NIR imaging revealed the localization of nanostructures in vitro and in vivo, which facilitated the determination of Pt(II) delivery to the target sites.

The four-armed amphiphilic Pt-PAZMBb-POEGMA copolymer, containing an aggregation-induced emissive fluorescent probe for imaging living cells and the anticancer drug 3,6-bis $\left[\right.$ trans-Pt $\left.\left(\mathrm{PEt}_{3}\right)_{2}\right]$ phenanthrene, was self-assembled into NPs, which were then used for encapsulate DOX to achieve a synergistic anticancer effect. ${ }^{280}$ 
A
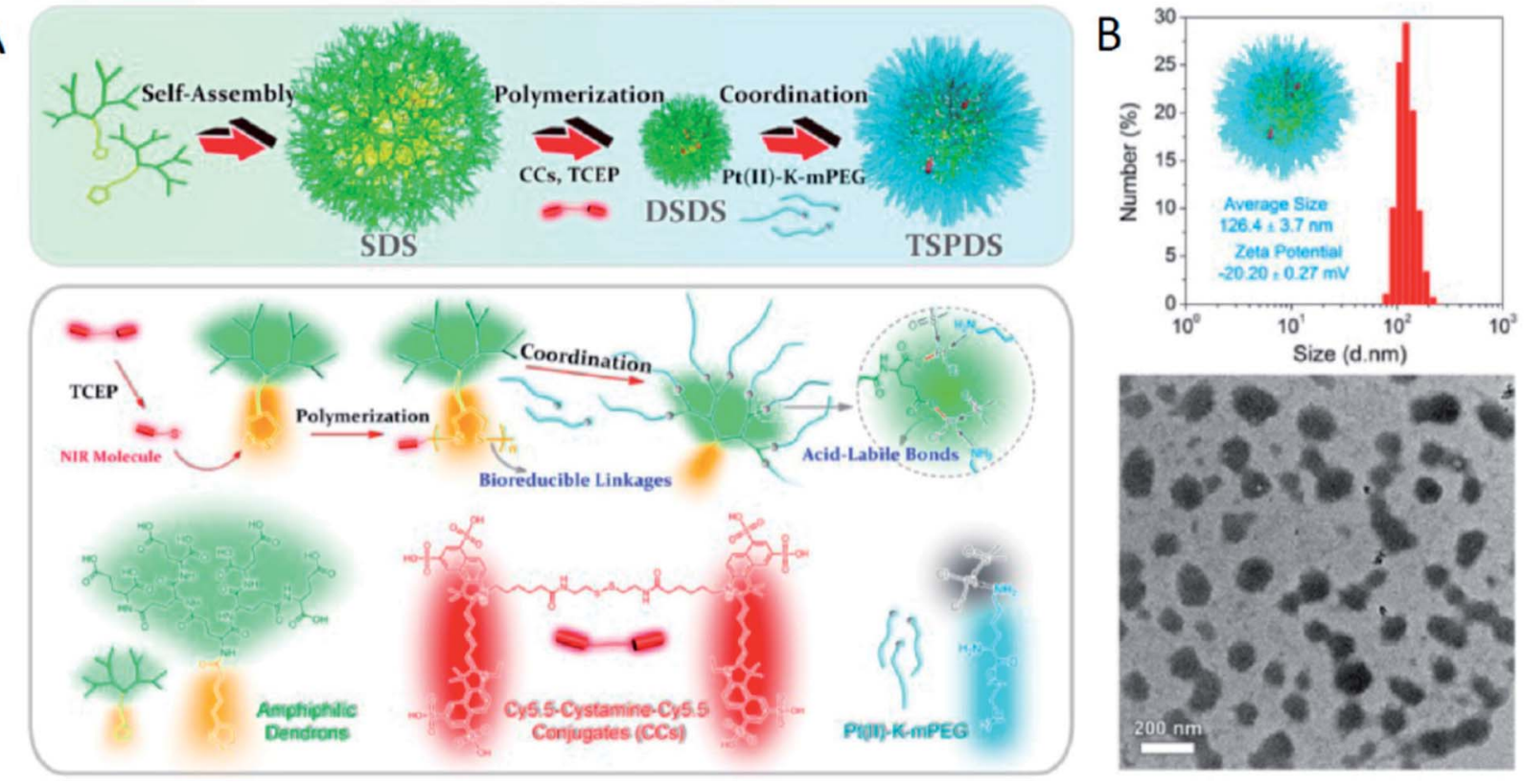

Fig. 46 (A) Schematic illustrations of molecular and supramolecular engineering of theranostic supramolecular PEGylated dendritic systems. (B) Dynamic Light Scattering (DLS) results (in aqueous solutions) and TEM images for theranostic supramolecular PEGylated dendritic systems (TSPDSs) [reprinted with permission from ref. 279. Copyright 2016, Ivyspring International Publisher].

Core-shell nanocomposites, based on the controlled growth of the UiO-66 shell on the surface of $\mathrm{Fe}_{3} \mathrm{O}_{4}$ core, have been developed for loading therapeutic drugs and simultaneous magnetic resonance imaging (MRI). ${ }^{281}$ Also noteworthy is a multifunctional nanoplatform based on $\mathrm{Fe}_{3} \mathrm{O}_{4}$ NPs, gold NPs and ZIF-8 NPs for integration chemotherapy and multimode imaging, including fluorescence imaging, MRI and X-ray computer tomography. Multifunctional NPs with many advantages, such as easy preparation conditions, a high degree of DOX separation, multimode imaging ability, pH-responsive drug release, and good biocompatibility, demonstrate effective anticancer performance and low systematic toxicity in in vivo studies. ${ }^{282}$ Of interest are MOFs, which not only include photosensitizers in periodic frameworks, but also have a high specific surface areas and large cavities for drug delivery. ${ }^{283}$ PDT is based on the excitation of photosensitizers in cancer tissues under light irradiation, which could efficiently produce ROS, causing lipid peroxidation, and increase the permeability of the cell membrane for irreversible killing of cancer cells.

A biocompatible fluorescent imaging-guided therapy system for cancer diagnosis and treatment was identified by integrating the properties of MOFs and chemotherapeutic drugs. ${ }^{284}$ Thus, biocompatible nanoscale zirconium-porphyrin MOFs-based system showed effective loading of DOX and a pH-responsive drug release for chemotherapy and a high porphyrin content to achieve effective PDT and fluorescent imaging. The imagingguided dual therapy system is confirmed by the concentration of nanomedicine in the cancer tissues, which leads to low physiological toxicity and effective cancer therapy.

Multifunctional MOFs, which serve as a drug carrier, quenching reagent, and post-synthetic modification site, have been obtained for synergistic chemo-photodynamic therapy and real-time cathepsin B-mediated fluorescence imaging. ${ }^{285}$

Porphyrin-based MOFs (PCNs) have been used to deliver tirapazamine (TPZ) of the bio-reductive drugs and are coated with cancer cell membrane (Mem) for homologous targeting of cancer (Fig. 47A). ${ }^{286}$ After exposure to light, PCN-mediated PDT can lead to oxygen consumption in cancer tissues, and the resulting regional hypoxia will additionally activate the bioreductive drugs of TPZ to improve chemotherapy (Fig. 47B). The synergistic effect of PDT and hypoxia-responsive chemotherapy in TPZ@PCN@Mem can significantly kill cancer cells and also suppress cancer metastasis.

Several methods have been developed to improve the anticancer efficacy of PTT and PTT-based synergistic therapies. For example, core-shell PB@MIL-100(Fe) dual metal-organicframeworks (d-MOFs) NPs, where PB is Prussian blue, can serve as a $T_{1}-T_{2}$ dual-modal MRI contrast and fluorescence optical imaging agent due to the presence of inner PB MOFs and outer MIL-100(Fe) MOFs. ${ }^{287}$ High-load artemisinin (a traditional Chinese anticancer drug) is released from d-MOFs during tumor cellular endocytosis due to $\mathrm{pH}$-responsive degradation of outer MOFs in low pH tumor cell lysosomes. In addition, the inner PB MOFs can be used for PTT due to its strong absorbance in the NIR region. In another interesting example, a simple and smart $\mathrm{pH} / \mathrm{NIR}$ dual-stimulus-responsive degradable mesoporous $\mathrm{CoFe}_{2} \mathrm{O}_{4} @ P D A @ Z I F-8$ sandwich nanocomposite, where PDA is polydopamine, was developed. The $\mathrm{CoFe}_{2} \mathrm{O}_{4}$ mesoporous core acts as T2-weighted MRI probe, PTT agent, and a hydrophilic DOX loading platform. The PDA layer is used to prevent premature leakage of DOX prior to arrival at tumor site, increase PTT efficiency, and facilitate ZIF-8 integration. The ZIF-8 shell 

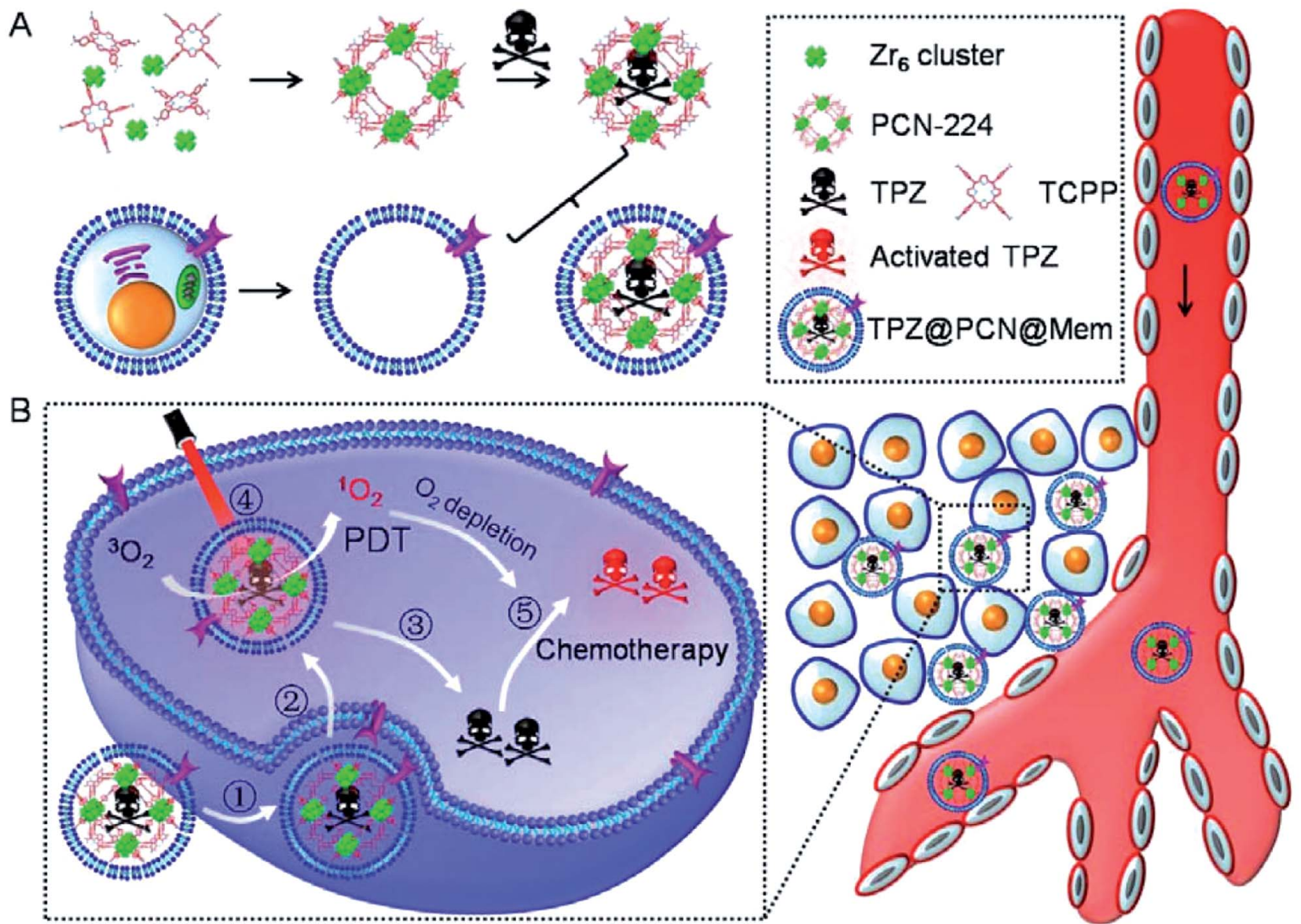

Fig. 47 (A) The process of manufacturing a porphyrin-based MOFs nanoplatform (TPZ@PCN@Mem). (B) Schematic diagram of TPZ@PCN@Mem for synergistic therapy of porphyrin-mediated PDT and hypoxia-induced chemotherapy [reprinted with permission from ref. 286 . Copyright 2017, Elsevier].

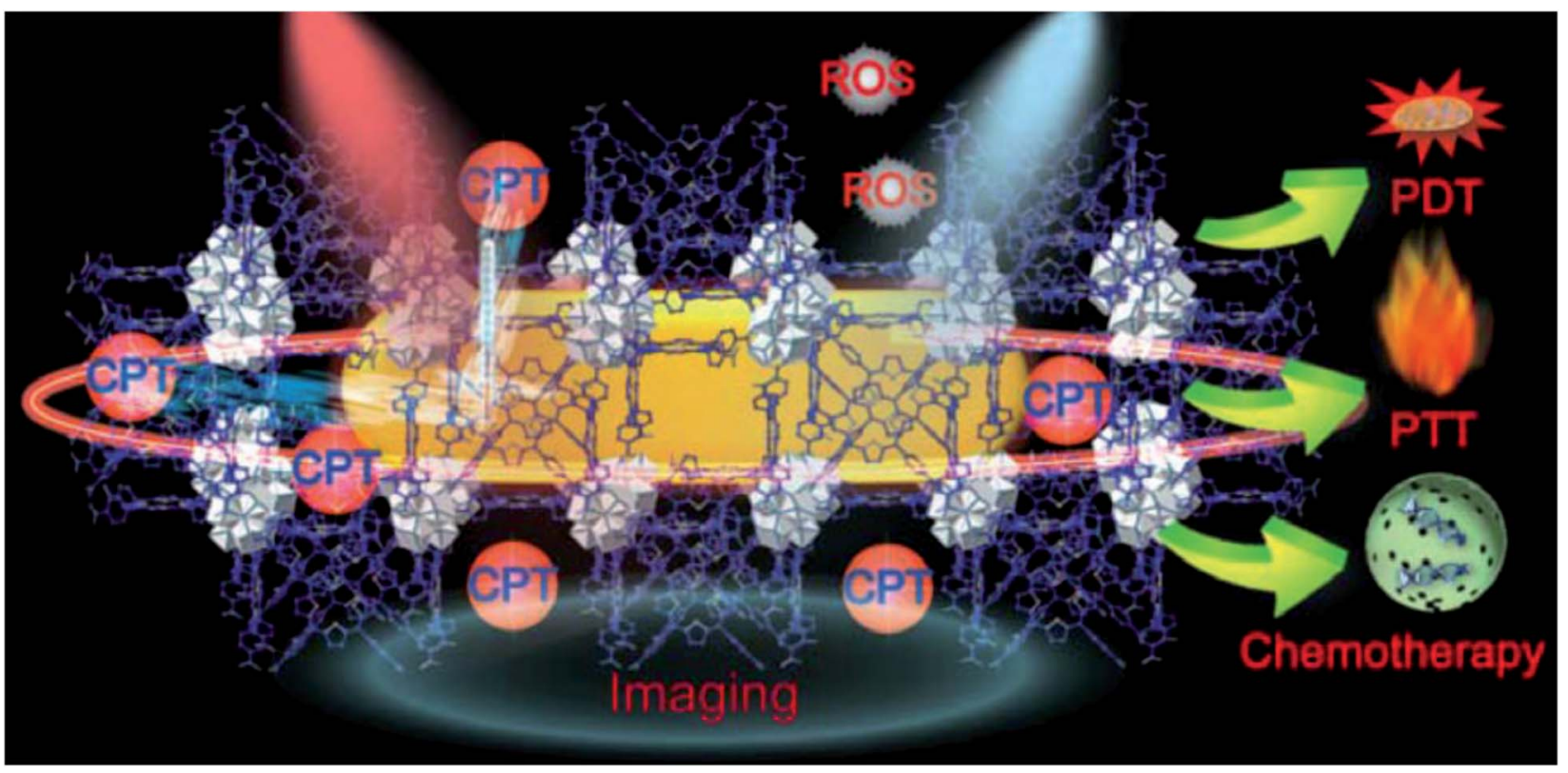

Fig. 48 Schematic representation of AuNR@MOFs loaded CPT for synergistic cancer therapy of chemotherapy/PDT/PTT [reprinted with permission from ref. 291. Copyright 2018, John Wiley and Sons]. 


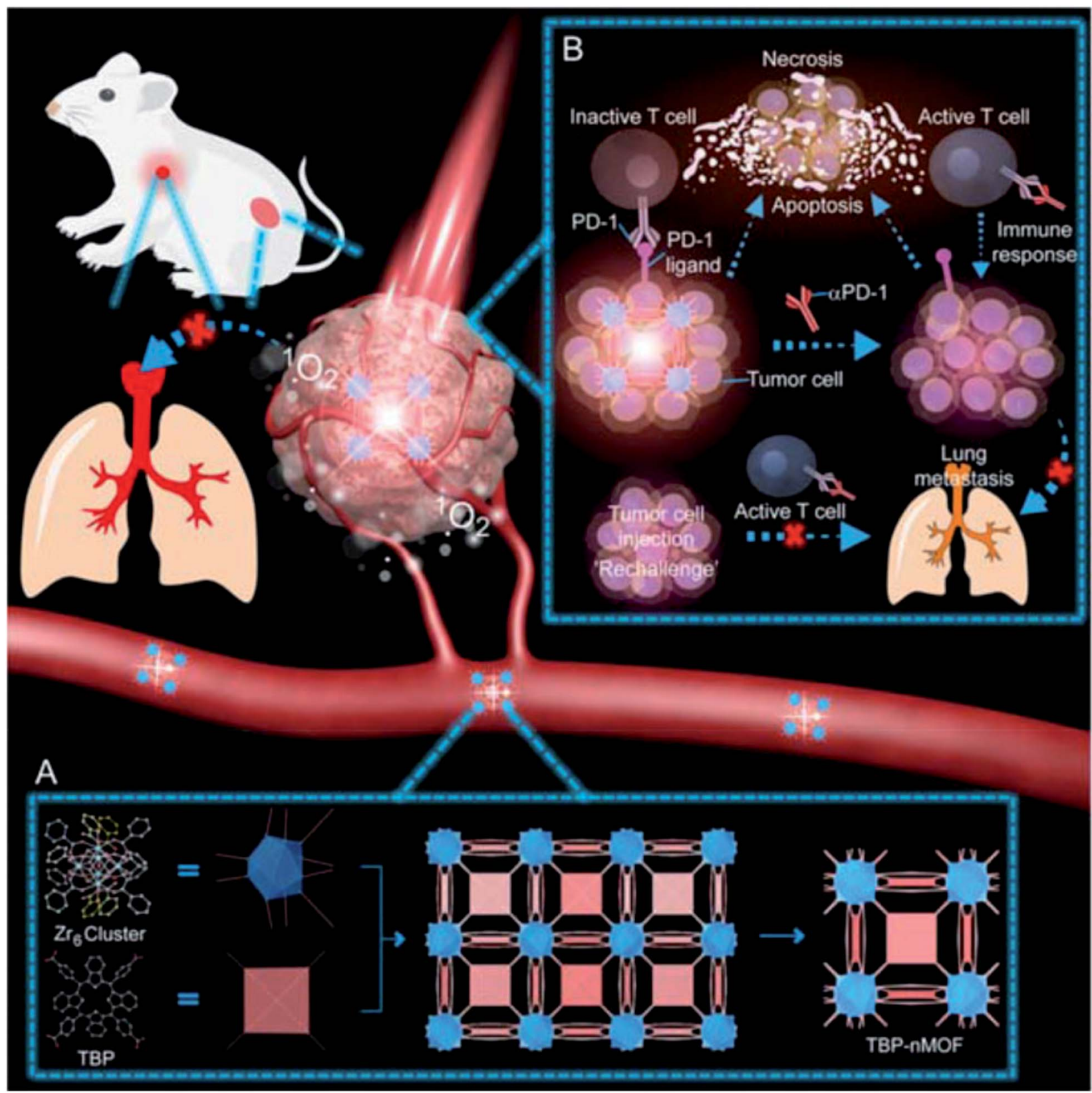

Fig. 49 (A) The preparation process of TBP-MOF. (B) Schematic presentation of TBP-MOFs-induced photodynamic cancer immunotherapy for effective inhibition of cancer metastasis [reprinted with permission from ref. 293. Copyright 2018, American Chemical Society].

serves to encapsulate hydrophobic CPT and as a switch for $\mathrm{pH}$ and NIR stimulation-responsive release of two drugs. ${ }^{288}$ Among other synergetic system using core-shell MOFs and organic PTT agents, we note PPy-based MOFs, PPy-MIL-100(Fe). The anticancer drug can be easily loaded into the mesopores of the MIL100 shell, and the PPy core, as an organic photothermal agent, can photothermally ablate cancer cells and improve the efficacy of chemotherapy under NIR irradiation. The nanocomposites demonstrated outstanding synergistic anticancer ability in vivo. ${ }^{289}$ The same PPy-MIL-100(Fe) was used for dual-mode MRI/ photoacoustic imaging and synergistic chemo-photothermal therapy of cancer cells. The multifunctional nanoplatform also demonstrated the ability to release a $\mathrm{pH} / \mathrm{NIR}$-responsive drug. ${ }^{290}$

In the core-shell porphyrin-based MOFs (AuNR@MOF), where AuNRs are gold nanorods, MOFs are used to load chemotherapeutic drugs such as CPT and diagnostic fluorescence imaging (Fig. 48). ${ }^{291}$ The AuNR core has an effective photothermal effect when irradiated with NIR light for PTT, which can also contribute to the release of therapeutic drugs for enhanced chemotherapy. In general, AuNR@MOFs have a significant synergistic effect for killing cancer cells and suppressing cancer recurrence and metastasis. 


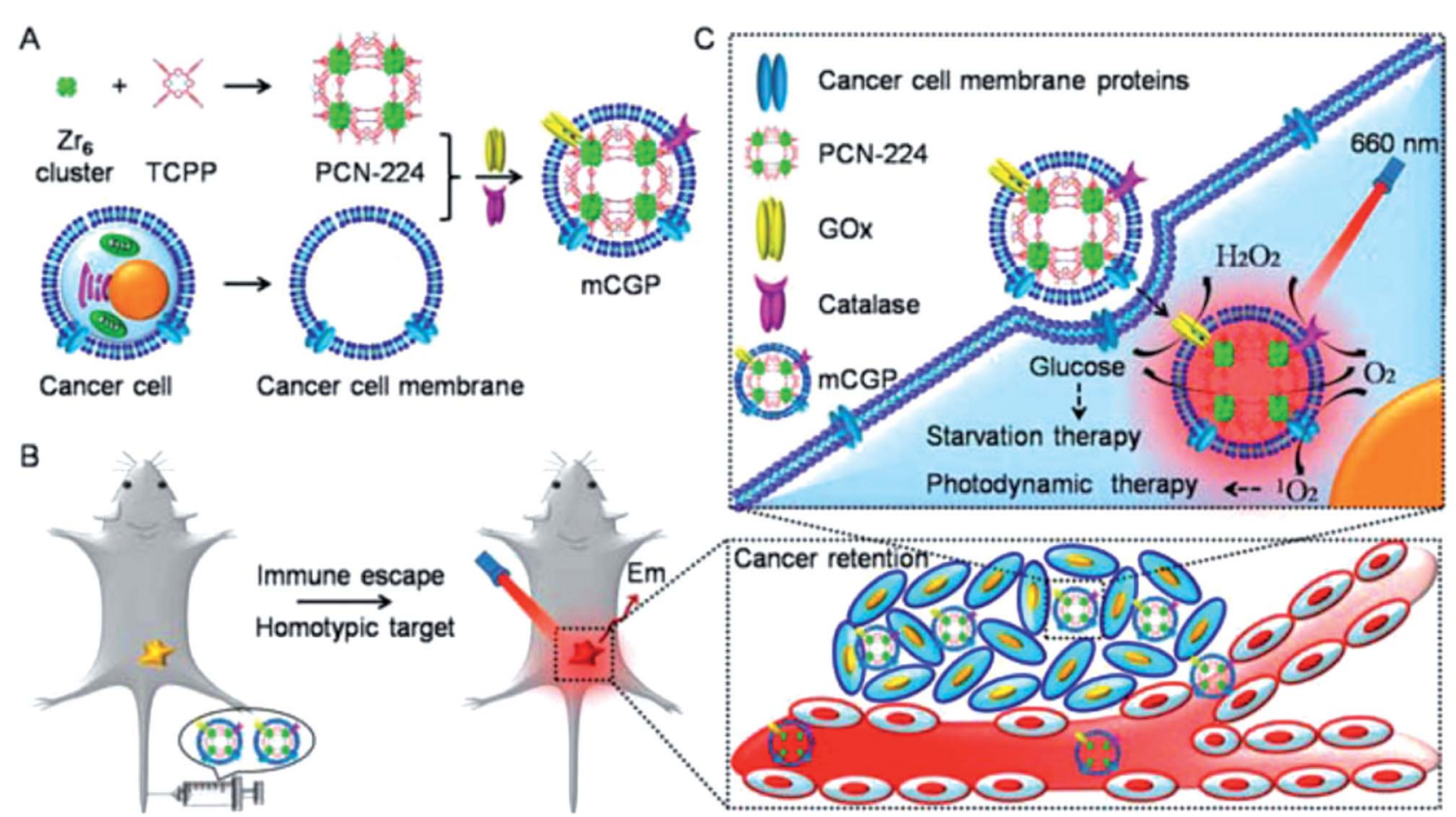

Fig. 50 (A) Processes for the preparation of PCN-224 as a nanocarrier for the delivery of GOx and catalase. (B) In vivo immune escape and homotypic cancer targeting of the composites. (C) Multifunctional MOFs-based nanoplatform for synergistic PDT and starvation therapy [reprinted with permission from ref. 294. Copyright 2018, American Chemical Society].

A strategy for synergistic cancer therapy based on a combination of chlorin-based MOFs mediated PDT and a small molecule immunotherapeutic agent encapsulated in the MOFs channels should be noted. ${ }^{\mathbf{2 9 2}}$ In this case, combination therapy can effectively inhibit indoleamine 2,3-dioxygenase and cause systemic anticancer immunity. In addition, this strategy can be effectively used to treat colorectal cancer.

Benzoporphyrin-based MOFs (TBP-MOF) effectively inhibit cancer metastasis based on the synergistic therapy of PDT and immunotherapy (Fig. 49A). ${ }^{293}$ To improve the physiological stability, TBP-MOF was modified by PEG, and PEG-modified TBP-MOF can conduct effective PDT in a hypoxic microenvironment. Due to the synergistic effect of TBP-MOF-mediated PDT and the $\alpha$ PD- 1 checkpoint blockade immunotherapy, primary cancer can be significantly suppressed, and cancer metastasis can also be efficiently inhibited (Fig. 49B).

Of interest are porphyrin-based MOFs (PCN-224) used as nanocarriers to deliver glucose oxidase (GOx) and catalase, which also served as nanophotosensitizer (Fig. 50A). The enzyme-loaded PCN-224 can be effectively used for synergistic PDT and starvation therapy (Fig. 50B and C). In addition, PCN224 , loaded with GOx and catalase, was further modified with a homotypic cancer cell membrane that could selectively target cancer tissue due to homotypic target talents. As a result, synergistic effects can efficiently kill cancer cells in a controlled manner. ${ }^{294}$

It should be noted the modification of panitumumab using MPs that are multiple DOTA chelators, providing stable and effective complexation of ${ }^{111}$ In or ${ }^{177} \mathrm{Lu}$, which was an EGFRtargeted theranostic for pancreatic cancer. ${ }^{295}$

Among the advantages of metallopolymers-based DDS with multimodality, such as imaging and targeted drug delivery, it should be noted a decrease in side effects, an improved targeting strategy and biodistribution, enhanced blood circulation and the penetration depth of existing body barriers, as well as a decrease in the required volume needed for distribution.

\section{Concluding remarks and outlook}

As the contents of this review show, the current stage of the development of metallopolymers-based DDSs has reached its peak in the accumulation of experimental facts and their theoretical interpretation and generalization; all major research groups are involved in this area of science. MPs showed great potential in DDSs due to their unique optical, electrochemical and magnetic properties. In addition, MPs participated in the codelivery of several chemotherapeutics drugs for the treatment of drug-resistant cancer. These functional DDSs have the potential to increase the solubility and efficacy of a drug while reducing side effects.

How we see development of this interesting and promising area of using metallopolymers?

First of all, it should be noted that the range of MPs used for these purposes is quite wide and is not limited to the examples given here. A huge selection of MPs with a wide arsenal of synthetic methods for their preparation, the simplicity of many formulations, scalability and an acceptable price/quality ratio 
provide a methodological platform to creating promising DDSs based on them. Among them, nanoscale MPs attract particular attention, in particular, for combination therapy. One promising area is the use of bioactive motifs (cations, organic ligands, or both) as building blocks for constructing metal biomolecule frameworks (also known as bioMOFs). A variety of hybrid materials, such as biopolymer@MOFs, are also of considerable interest. It is important to develop MPs whose polymeric chains include non-traditional heteroatoms, for example, selenium or tellurium. Stimuli-responsive metallopolymers-based DDSs are one of the most promising class of nanoplatforms that meet the requirements of an effective chemotherapeutic drug formulation.

Despite their enormous potential, very few metallopolymersbased DDSs have been included in clinical trials and no one has yet been approved for the treatment of the disease. Indeed, there are still some problems that must be overcome in order to begin the effective pharmaceutical development of such systems. Unfortunately, for many metallopolymers-based DDSs the exact mechanism of action and behavior in the biological environment are not fully understood. Another major challenge is the assessment of systemic toxicity and in vivo pharmacokinetics. Although a large number of metallopolymer-based DDSs have been studied in vitro with varying cytotoxicity, little is known about their absorption, distribution, metabolism, and excretory properties in humans. Further problems arise due to the requirements for precise control and initiation of the responsive systems. In addition, till now, as a rule, it is impossible to recognize correlations between content, structural features, and properties of metallopolymer-based DDSs, which in many respects restrains scientifically grounded approach to structuring of these systems and prediction of their promising properties.

\section{Conflicts of interest}

No potential conflict of interest was reported by the authors.

\section{Acknowledgements}

This work was performed by Gulzhian I. Dzhardimalieva (Institute of Problems of Chemical Physics RAS) in accordance with the state task, state registration No. 0089-2019-0008.

\section{References}

1 J.-A. Martyn, P. Paliadelis and C. Perry, Nurse Educ. Pract., 2019, 37, 109-114.

2 S. S. Dhruva, J. S. Ross and N. R. Desai, Pharmacy and Therapeutics (P\&T), 2018, 43, 464-472.

3 Y.-C. Kim, K. A. Min, D.-J. Jang, T. Y. Ahn, J. H. Min, B. E. Yu and K. H. Cho, J. Pharm. Invest., DOI: 10.1007/s40005-01900452-0.

4 A. D. Vadlapudi, D. Kwatra and A. K. Mitra, in Drug Delivery, ed. A. K. Mitra, D. Kwatra and A. D. Vadlapudi, Jones \& Bartlett Learning, Burlington, USA, 2015, pp. 1-7.
5 Y. Duarte, V. Márquez-Miranda, M. J. Miossec and F. González-Nilo, Wiley Interdiscip. Rev.: Nanomed. Nanobiotechnol., 2019, 11, e1554.

6 R. Spitler, S. Zanganeh, T. Jafari, N. Khakpash, M. Erfanzadeh, J. Q. Ho and N. Sakhaie, in Drug Delivery Systems, ed. P. Stroeve and M. Mahmoudi, World Scientific Publ. Co., New Jersey, 2018, pp. 1-52.

7 C. He, D. Liu and W. Lin, Chem. Rev., 2015, 115, 1107911108.

8 N. Song and Y. W. Yang, Chem. Soc. Rev., 2015, 44, 34743504.

9 A. A. Moghanjoughi, D. Khoshnevis and A. Zarrabi, Drug Delivery Transl. Res., 2016, 6, 333-340.

10 M. Daniyal, B. Liu and W. Wang, Curr. Med. Chem., DOI: 10.2174/13816128256661902011296290.

11 X. Zhao, K. Tian, T. Zhou, X. Jia, J. Li and P. Liu, Colloids Surf., B, 2018, 168, 43-49.

12 J. Wang, J. Fang, P. Fang, X. Li, S. Wu, W. Zhang and S. Li, J. Biomater. Sci., Polym. Ed., 2017, 28, 337-349.

13 M. M. Goswami, Sci. Rep., 2016, 6, 35721.

14 A. Llopis-Lorente, B. Lozano-Torres, A. Bernardos, R. Martínez-Máñez and F. Sancenón, J. Mater. Chem. B, 2017, 5, 3069-3083.

15 H. He, H. Xiao, H. Kuang, Z. Xie, X. Chen, X. Jing and Y. Huang, Colloids Surf., B, 2014, 117, 75-81.

16 M. Yilmaz, A. A. Karanastasis, M. V. Chatziathanasiadou, M. Oguz, A. Kougioumtzi, N. Clemente, T. F. Kellici, N. E. Zafeiropoulos, A. Avgeropoulos, T. Mavromoustakos, U. Dianzani, S. Karakurt and A. G. Tzakos, ACS Appl. Bio Mater., 2019, 2, 2715-2725.

17 K. Sztandera, M. Gorzkiewicz and B. Klajnert-Maculewicz, Mol. Pharm., 2019, 16, 1-23.

18 S. Perfahl, M. M. Natile, H. S. Mohamad, C. A. Helm, C. Schulzke, G. Natile and P. J. Bednarski, Mol. Pharmacol., 2016, 13, 2346-2362.

19 X. Wang, C. Liu, Z. Li, C.-Y. Tang, W.-C. Law, X. Gong, Z. Liu, Y. Liao, G. Zhang, S. Long and L. Chen, J. Phys. Chem. C, 2019, 123, 10658-10665.

20 H. Pick, A. C. Alves and H. Vogel, Chem. Rev., 2018, 118, 8598-8654.

21 S. Genheden and L. A. Eriksson, J. Chem. Theory Comput., 2016, 12, 4651-4661.

22 M. M. Virk and E. Reimhult, Langmuir, 2018, 34, 395-405.

23 P. Ghasemiyeh and S. Mohammadi-Samani, Results Pharma Sci., 2018, 13, 288-303.

24 M. Rawal, in Stimuli-responsive Drug Delivery Systems, ed. A. Singh and M. M. Amiji, Royal Society of Chemistry, London, 2018, pp. 33-50.

25 S. Indermun, M. Govender, P. Kumar, Y. E. Choonara and V. Pillay, in Stimuli Responsive Polymeric Nanocarriers for Drug Delivery Applications, ed. A. S. H. Makhlouf and N. Y. Abu-Thabit, Elsevier, Amsterdam, 2019, pp. 43-58.

26 N. Kamaly, B. Yameen, J. Wu and O. C. Farokhzad, Chem. Rev., 2016, 116, 2602-2663.

27 P. R. Nair, Polymers, 2019, 11, 630.

28 A. Wicki, D. Witzigmann, V. Balasubramanian and J. Huwyler, J. Controlled Release, 2015, 200, 138-157. 
29 T. Stylianopoulos and R. K. Jain, Nanomedicine, 2015, 11, 1893-1907.

30 Z. Li and Y.-W. Yang, J. Mater. Chem. B, 2017, 5, 9278-9290.

31 M.-X. Wu, X. Wang and Y.-W. Yang, Chem. Rec., 2018, 18, 45-54.

32 K. Ulbrich, K. Holá, V. Šubr, A. Bakandritsos, J. Tuček and R. Zbořil, Chem. Rev., 2016, 116, 5338-5431.

33 W. Chin, G. Zhong, Q. Pu, C. Yang, W. Lou, P. F. De Sessions, B. Periaswamy, A. Lee, Z. C. Liang, X. Ding, S. Gao, C. W. Chu, S. Bianco, C. Bao, Y. W. Tong, W. Fan, M. Wu, J. L. Hedrick and Y. Y. Yang, Nat. Commun., 2018, 9, 917.

34 G. I. Dzhardimalieva and I. E. Uflyand, Chemistry of Polymeric Metal Chelates, Springer, Cham, 2018.

35 G. I. Dzhardimalieva and I. E Uflyand, J. Inorg. Organomet. Polym. Mater., 2018, 28, 1305-1393.

36 R. L. N. Hailes, A. M. Oliver, J. Gwyther, G. R. Whittell and I. Manners, Chem. Soc. Rev., 2016, 45, 5358-5407.

37 Y. Wang, D. Astruc and A. S. Abd-El-Aziz, Chem. Soc. Rev., 2019, 48, 558-636.

38 K. Y. Zhang, S. Liu, Q. Zhao and W. Huang, Coord. Chem. Rev., 2016, 319, 180-195.

39 K. C. Bentz and S. M. Cohen, Angew. Chem., Int. Ed., 2018, 57, 14992-15001.

40 H. Li, P. Yang, P. Pageni and C. Tang, Macromol. Rapid Commun., 2017, 38, 1700109.

41 R. A. Musgrave, A. D. Russell, D. W. Hayward, G. R. Whittell, P. G. Lawrence, P. J. Gates, J. C. Green and I. Manners, Nat. Chem., 2017, 9, 743-750.

42 M. O. Wolf, Adv. Mater., 2001, 13, 545-553.

43 M. O. Wolf, J. Inorg. Organomet. Polym. Mater., 2006, 16, 189-199.

44 A. Winter and U. S. Schubert, Chem. Soc. Rev., 2016, 45, 5311-5357.

45 A. Abd-El-Aziz and C. Agatemor, J. Inorg. Organomet. Polym. Mater., 2018, 28, 369-382.

46 T. Zhu, Y. Sha, J. Yan, P. Pageni, M. A. Rahman, Y. Yan and C. Tang, Nat. Commun., 2018, 9, 4329.

47 L. Zhao, X. Liu, L. Zhang, G. Qiu, D. Astruc and H. Gu, Coord. Chem. Rev., 2017, 337, 34-79.

48 X. Wang, X. Wang and Z. Guo, Acc. Chem. Res., 2015, 48, 2622-2631.

49 J. Xiang, C. L. Ho and W. Y. Wong, Polym. Chem., 2015, 6, 6905-6930.

50 C.-L. Ho, Z.-Q. Yu and W.-Y. Wong, Chem. Soc. Rev., 2016, 45, 5264-5295.

51 A. Alkan and F. R. Wurm, Macromol. Rapid Commun., 2016, 37, 1482-1493.

52 T. Zhu, S. Xu, A. Rahman, E. Dogdibegovic, P. Yang, P. Pageni, M. P. Kabir, X.-d. Zhou and C. Tang, Angew. Chem., Int. Ed., 2018, 57, 2388-2392.

53 A. S. Abd-El-Aziz, C. Agatemor and N. Etkin, in Functional Metallosupramolecular Materials, ed. J. Hardy and F. Schacher, RSC Publishing, London, 2015, pp. 87-119.

54 J.-F. Lutz, J.-M. Lehn, E. W. Meijer and K. Matyjaszewski, Nat. Rev. Mater., 2016, 1, 16024.
55 R. Sakamoto, K.-H. Wu, R. Matsuoka, H. Maeda and H. Nishihara, Chem. Soc. Rev., 2015, 44, 7698-7714.

56 D. A. Pelletier and J. M. Basset, Acc. Chem. Res., 2016, 49, 664-677.

57 O. Pamies, M. Dieguez and J.-E. Backvall, Adv. Synth. Catal., 2015, 357, 1567-1586.

58 M. Hoarau, C. Hureau, E. Gras and P. Faller, Coord. Chem. Rev., 2016, 308, 445-459.

59 D. F. Sauer, S. Gotzen and J. Okuda, Org. Biomol. Chem., 2016, 14, 9174-9183.

60 Y. W. Lin, Coord. Chem. Rev., 2017, 336, 1-27.

61 K. Wieszczycka and K. Staszak, Coord. Chem. Rev., 2017, 351, 160-171.

62 F. Schwizer, Y. Okamoto, T. Heinisch, Y. Gu, M. M. Pellizzoni, V. Lebrun, R. Reuter, V. Köhler, J. C. Lewis and T. R. Ward, Chem. Rev., 2018, 118, 142-231.

63 H.-C. Chang, J.-Q. Li, C.-K. Lin, Y.-J. Hsu, T.-H. Tu, Y.-L. Hsieh, H.-H. Hsu, G.-H. Lee, Y.-H. Liu and C.-H. Peng, J. Chin. Chem. Soc., 2019, 66, 1119-1133.

64 A. S. Abd-El-Aziz, A. A. Abdelghani, B. D. Wagner and R. Bissessur, Macromol. Rapid Commun., 2018, 40, 1800711.

65 J. D. Caraway, M. T. Nguyen, L. A. Mitchell and B. J. Holliday, Macromol. Rapid Commun., 2015, 36, 665670.

66 M. Mauro, S. Bellemin-Laponnaz and C. Cebrian, Chem.Eur. J., 2017, 23, 17626-17636.

67 K. Albrecht, K. Matsuoka, K. Fujita and K. Yamamoto, Angew. Chem., Int. Ed., 2015, 54, 5677-5682.

68 M. Gallei and C. Rüttiger, Chem.-Eur. J., 2018, 24, 1000610021.

69 Q. He, C. Wang, J. Xiao, Y. Wang, Y. Zhou, N. Zheng, B. Zhang and W. Bu, J. Mater. Chem. C, 2018, 6, 1218712191.

70 E. Hobbollahi, M. List and U. Monkowius, Monatsh. Chem., 2019, 150, 877-883.

71 M. T. Nguyen, R. A. Jones and B. J. Holliday, Coord. Chem. Rev., 2018, 377, 237-258.

72 F. Lu and D. Astruc, Coord. Chem. Rev., 2018, 356, 147-164.

73 I. E. Uflyand and G. I. Dzhardimaleva, Nanomaterials Preparation by Thermolysis of Metal Chelates, Springer, Cham, 2018.

74 M. Komiyama, T. Mori and K. Ariga, Bull. Chem. Soc. Jpn., 2018, 91, 1075-1111.

75 A. R. Kenaree and J. B. Gilroy, Dalton Trans., 2016, 45, 18229-18240.

76 S.-C. Yiu, C.-L. Ho and W.-Y. Wong, in Polymer-Engineered Nanostructures for Advanced Energy Applications, ed. Z. Lin, Y. Yang and A. Zhang, Springer, Cham, 2017, pp. 51-70.

77 J. Gopinath, R. K. C. Balasubramanyam, V. Santosh, S. K. Swami, D. K. Kumar, S. K. Gupta, V. Dutta, K. R. Reddy, V. Sadhu, A. V. S. Sainath and T. M. Aminabhavi, Chem. Eng. J., 2019, 358, 1166-1175.

78 M. J. Dunlop, C. Agatemor, A. S. Abd-El-Aziz and R. Bissessur, J. Inorg. Organomet. Polym. Mater., 2017, 27, 84-89.

79 Q. Dong, Z. Meng, C.-L. Ho, H. Guo, W. Yang, I. Manners, L. Xu and W.-Y. Wong, Chem. Soc. Rev., 2018, 47, 4934-4953. 
80 Q. Dong, W. Qu, W. Liang, F. Tai, K. Guo, C.-W. Leung, Y. H. Lo and W.-Y. Wong, J. Mater. Chem. C, 2016, 4, 5010-5018.

81 S. Schubert, I. Manners, G. R. Newkome and U. S. Schubert, Macromol. Rapid Commun., 2015, 36, 585.

82 S. G. Song, S. Ha, K.-B. Seo, J. Lee, T.-L. Choi, T. Premkumar and C. Song, RSC Adv., 2016, 6, 107994-107999.

83 A. Alkan, T. Gleede and F. R. Wurm, Organometallics, 2017, 36, 3023-3028.

84 C. C. Beto, E. D. Holt, Y. Yang, I. Ghiviriga, K. S. Schanze and A. S. Veige, Chem. Commun., 2017, 53, 9934-9937.

85 C. Rüttiger, H. Hübner, S. Schöttner, T. Winter, G. Cherkashinin, B. Kuttich, B. Stühn and M. Gallei, ACS Appl. Mater. Interfaces, 2018, 10, 4018-4030.

86 L. Babel, K. Baudet, T. N. Y. Hoang, H. Nozary and C. Piguet, Chem.-Eur. J., 2018, 24, 5423-5433.

87 G. R. Newkome, C. N. Moorefield and S. Chakraborty, J. Inorg. Organomet. Polym. Mater., 2018, 28, 360-368.

88 I. Dragutan, V. Dragutan, B. C. Simionescu, A. Demonceau and H. Fischer, Beilstein J. Org. Chem., 2015, 11, 2747-2762.

89 P. Yang, P. Pageni, M. P. Kabir, T. Zhu and C. Tang, ACS Macro Lett., 2016, 5, 1293-1300.

90 L. Babel, L. Guénée, C. Besnard, S. V. Eliseeva, S. Petoud and C. Piguet, Chem. Sci., 2018, 9, 325-335.

91 X. Liu, Q. Ling, L. Zhao, G. Qiu, Y. Wang, L. Song, Y. Zhang, J. Ruiz, D. Astruc and H. Gu, Macromol. Rapid Commun., 2017, 38, 1700448.

92 C. Rüttiger, M. Appold, H. Didzoleit, A. Eils, C. Dietz, R. W. Stark, B. Stühn and M. Gallei, Macromolecules, 2016, 49, 3415-3426.

93 A. Rahman, Y. Cha, L. Yuan, P. Pageni, T. Zhu, M. S. Jui and C. Tang, J. Polym. Sci., Part A: Polym. Chem., DOI: 10.1002/ pola.29439.

94 T. Imaoka and K. Yamamoto, Bull. Chem. Soc. Jpn., 2019, 92, 941-948.

95 P. Kumar, K. Vellingiri, K.-H. Kim, R. J. C. Brown and M. J. Manos, Microporous Mesoporous Mater., 2017, 253, 251-265.

96 C. Pettinari, F. Marchetti, N. Mosca, G. Tosi and A. Drozdov, Polym. Int., 2017, 66, 731-744.

97 M. J. Rosseinsky, M. W. Smith and C. M. Timperley, Nat. Mater., 2015, 14, 469-470.

98 Q. Yang, Q. Xu and H. L. Jiang, Chem. Soc. Rev., 2017, 46, 4774-4808.

99 W. P. Lustig, S. Mukherjee, N. D. Rudd, A. V. Desai, J. Li and S. K. Ghosh, Chem. Soc. Rev., 2017, 46, 3242-3285.

100 Y. Liu, S. Zhou, S. Liu and M. Xu, ChemElectroChem, 2018, 5, 6-19.

101 L. Zhu, X. Q. Liu, H. L. Jiang and L. B. Sun, Chem. Rev., 2017, 117, 8129-8176.

102 W. Wang, X. Xu, W. Zhou and Z. Shao, Adv. Sci., 2017, 4, 1600371.

103 K. K. Gangu, S. Maddila, S. B. Mukkamala and S. B. Jonnalagadda, Inorg. Chim. Acta, 2016, 446, 61-74.

104 Y. Cui, B. Li, H. He, W. Zhou, B. Chen and G. Qian, Acc. Chem. Res., 2016, 49, 483-493.
105 C. F. Cogswell, Z. Xie and S. Choi, in Metal-Organic Frameworks: Applications in Separations and Catalysis, ed. H. García and S. Navalón, Wiley, Weinheim, 2018, pp. 297-340.

106 G. Maurin, C. Serre, A. Cooper and G. Férey, Chem. Soc. Rev., 2017, 46, 3104-3107.

107 W. Cai, C. C. Chu, G. Liu and Y. X. Wang, Small, 2015, 11, 4806-4822.

108 Y. Sun and H. C. Zhou, Adv. Mater., 2015, 16, 054202.

109 B. Seoane, S. Castellanos, A. Dikhtiarenko, F. Kapteijn and J. Gascon, Coord. Chem. Rev., 2016, 307, 147-187.

110 K. E. Cordova and O. M. Yaghi, Mater. Chem. Front., 2017, 1, 1304-1309.

111 C.-C. Wang, S.-Y. Ke, K.-T. Chen, Y.-F. Hsieh, T.-H. Wang, G.-H. Lee and Y.-C. Chuang, in Structural Design and Properties of Coordination Polymers, ed. G. E. Kostakis, MDPI, Basel, 2018, pp. 1-12.

112 G. I. Dzhardimalieva and I. E. Uflyand, $R S C A d v ., 2017,7$, $42242-42288$.

113 V. V. Butova, M. A. Soldatov, A. A. Guda, K. A. Lomachenko and C. Lamberti, Russ. Chem. Rev., 2016, 85, 280-307.

114 Y. Yan, J. Zhang, L. Ren and C. Tang, Chem. Soc. Rev., 2016, 45, 5232-5263.

115 L. Thomi, P. Schaefer, K. Landfester and F. R. Wurm, Macromolecules, 2015, 45, 105-109.

116 A. Zhang, A. Li, W. Zhao and J. Liu, Chem.-Eur. J., 2018, 24, 4215-4227.

117 H. Gu, S. Mu, G. Qiu, X. Liu, L. Zhang, Y. Yuan and D. Astruc, Coord. Chem. Rev., 2018, 364, 51-85.

118 X. He, X. He, S. Li, K. Zhuo, W. Qin, S. Dong, J. Chen, L. Ren, G. Liu and H. Xia, Polym. Chem., 2017, 8, 3674-3678.

119 Z. Lu, Y. Cai, Y. Wei, Q. Lin, J. Chen, X. He, S. Li, W. Wu and H. Xia, Polym. Chem., 2018, 9, 2092-2100.

120 Z. Lu, Q. Lin, Y. Cai, S. Chen, J. Chen, W. Wu, X. He and H. Xia, ACS Macro Lett., 2018, 7, 1034-1038.

121 E. F. Panarin, Russ. Chem. Bull., 2017, 66, 1812-1820.

122 S. Kargozar, S. Ramakrishna and M. Mozafari, Current Opinion in Biomedical Engineering, 2019, 10, 181-190.

123 P. Jain, G. Pandey, D. Kumar and S. Chandra, Adv. Sci., Eng. Med., 2019, 11, 144-154.

124 H. Xiao, L. Yan, E. M. Dempsey, W. Song, R. Qi, W. Li, Y. Huang, X. Jing, D. Zhou, J. Ding and X. Chen, Prog. Polym. Sci., 2018, 87, 70-106.

125 M. Giménez-Marqués, T. Hidalgo, C. Serre and P. Horcajada, Coord. Chem. Rev., 2016, 307, 342-360.

126 M. X. Wu and Y. W. Yang, Adv. Mater., 2017, 29, 1606134.

127 G. Parekh, Y. Shi, J. Zheng, X. Zhang and S. Leporatti, Ther. Delivery, 2018, 9, 451-468.

128 L. He, Y. Liu, J. Lau, W. Fan, Q. Li, C. Zhang, P. Huang and X. Chen, Nanomedicine, 2019, 14, 1343-1365.

129 Y. Zhang, Q. Wang, G. Chen and P. Shi, Inorg. Chem., 2019, 58, 6593-6596.

130 A. A. Simagina, M. V. Polynski, A. V. Vinogradov and E. A. Pidko, Russ. Chem. Rev., 2018, 87, 831-858.

131 Y. Liu, Y. Zhao and X. Chen, Theranostics, 2019, 9, 31223133. 
132 Z. Zhang, W. Sang, L. Xie and Y. Dai, Coord. Chem. Rev., 2019, 399, 213022.

133 L. Côrte-Real, B. Karas, P. Gírio, A. Moreno, F. Avecilla, F. Marques, B. T. Buckley, K. R. Cooper, C. Doherty, P. Falson, M. H. Garcia and A. Valente, Eur. J. Med. Chem., 2019, 163, 853-863.

134 L. Côrte-Real, M. P. Robalo, F. Marques, G. Nogueira, F. Avecilla, T. J. L. Silva, F. C. Santos, A. I. Tomaz, M. H. Garcia and A. Valente, J. Inorg. Biochem., 2015, 150, 148-159.

135 C. Shi, H. Yu, D. Sun, L. Ma, Z. Tang, Q. Xiao and X. Chen, Acta Biomater., 2015, 18, 68-76.

136 H. Yu, Z. Tang, D. Zhang, W. Song, Y. Zhang, Y. Yang, Z. Ahmad and X. Chen, J. Controlled Release, 2015, 205, 89-97.

137 N. A. N. Hanafy, M. El-Kemary and S. Leporatti, Cancers, 2018, 10, 238.

138 S. Biswas, P. Kumari, P. M. Lakhani and B. Ghosh, Eur. J. Pharm. Sci., 2016, 83, 184-202.

139 J. Ahn, Y. Miura, N. Yamada, T. Chida, X. Liu, A. Kim, R. Sato, R. Tsumura, Y. Koga, M. Yasunaga, N. Nishiyama, Y. Matsumura, H. Cabral and K. Kataoka, Biomaterials, 2015, 39, 23-30.

140 M. Lu, F. Chen, J. M. Noy, H. Lu and M. H. Stenzel, Macromol. Biosci., 2017, 17, 1600513.

141 M. A. Miller, B. Askevold, H. Mikula, R. H. Kohler, D. Pirovich and R. Weissleder, Nat. Commun., 2017, 8, 15906.

142 S. C. Larnaudie, J. C. Brendel, I. Romero-Canelón, C. Sanchez-Cano, S. Catrouillet, J. Sanchis, J. P. C. Coverdale, J.-I. Song, A. Habtemariam, P. J. Sadler, K. A. Jolliffe and S. Perrier, Biomacromolecules, 2018, 19, 239-247.

143 W. Shen, J. Luan, L. Cao, J. Sun, L. Yu and J. Ding, Biomacromolecules, 2015, 16, 105-115.

144 Z. Zhang, Y. Wu, G. Kuang, S. Liu, D. Zhou, X. Chen, X. Jing and Y. Huang, J. Mater. Chem. B, 2017, 5, 2115-2125.

145 Z. Zhang, Y. Li, J. Wan, P. Long, J. Guo, G. Chen and C. Wang, Polym. Chem., 2017, 8, 2410-2422.

146 Y. Zhang, T. Sun and C. Jiang, Acta Pharm. Sin. B, 2018, 8, 34-50.

147 M. T. Wlodarczyk, S. A. Dragulska, O. Camacho-Vanegas, P. R. Dottino, A. A. Jarzęcki, J. A. Martignetti and A. J. Mieszawska, ACS Biomater. Sci. Eng., 2018, 4, 463-467.

148 N. Gupta, J. Kancharla, S. Kaushik, A. Ansari, S. Hossain, R. Goyal, M. Pandey, J. Sivaccumar, S. Hussain, A. Sarkar, A. Sengupta, S. K. Mandal, M. Roy and S. Sengupta, Chem. Sci., 2017, 8, 2387-2395.

149 E. J. Cho, B. Sun, K. O. Doh, E. M. Wilson, S. TorregrosaAllen, B. D. Elzey and Y. Yeo, Biomaterials, 2015, 37, 312319.

150 S. Liang, Y. Duan, J. Zhang, Z. Xing, X. Chen, Y. Yang and Q. Li, J. Controlled Release, 2015, 213, e95-e96.

151 X. Ling, Y. Shen, R. Sun, M. Zhang, C. Li, J. Mao, J. Xing, C. Sun and J. Tu, Polym. Chem., 2015, 6, 1541-1552.

152 W. Zhang, Z. Zhang and C. H. Tung, Chem. Commun., 2017, 53, 779-782.
153 M. S. Gil, T. Thambi, V. H. G. Phan, S. H. Kim and D. S. Lee, J. Mater. Chem. B, 2017, 5, 7140-7152.

154 C. He, C. Poon, C. Chan, S. D. Yamada and W. Lin, J. Am. Chem. Soc., 2016, 138, 6010-6019.

155 C. Poon, X. Duan, C. Chan, W. Han and W. Lin, Mol. Pharmaceutics, 2016, 13, 3665-3675.

156 Y. Yang, L. Xu, W. Zhu, L. Feng, J. Liu, Q. Chen, Z. Dong, J. Zhao, Z. Liu and M. Chen, Biomaterials, 2018, 156, 121133.

157 A. B. Lago, A. Pino-Cuevas, R. Carballo and E. M. VázquezLópez, Dalton Trans., 2016, 45, 1614-1621.

158 M. Rezaei, A. Abbasi, R. Dinarvand, M. Jeddi-Tehrani and J. Janczak, ACS Appl. Mater. Interfaces, 2018, 10, 1759417604.

159 J.-Y. Zeng, X.-S. Wang, W.-F. Song, H. Cheng and X.-Z. Zhang, Adv. Ther., 2019, 2, 1800100.

160 T. Simon-Yarza, A. Mielcarek, P. Couvreur and C. Serre, Adv. Mater., 2018, 30, 1707365.

161 S. Z. Wang, C. M. McGuirk, A. d'Aquino, J. A. Mason and C. A. Mirkin, Adv. Mater., 2018, 30, 1800202.

162 W. Chen and C. S. Wu, Dalton Trans., 2018, 47, 2114-2133.

163 S. Wuttke, M. Lismont, A. Escudero, B. Rungtaweevoranit and W. J. Parak, Biomaterials, 2017, 123, 172-183.

164 R. Freund, U. Lächelt, T. Gruber, B. Rühle and S. Wuttke, ACS Nano, 2018, 12, 2094-2105.

165 S. K. Alsaiari, S. Patil, M. Alyami, K. O. Alamoudi, F. A. Aleisa, J. S. Merzaban, M. Li and N. M. Khashab, J. Am. Chem. Soc., 2017, 140, 143-149.

166 R. Röder, T. Preiß, P. Hirschle, B. Steinborn, A. Zimpel, M. Höhn, J. O. Rädler, T. Bein, E. Wagner, S. Wuttke and U. Lächelt, J. Am. Chem. Soc., 2017, 139, 2359-2368.

167 T. Simon-Yarza, M. Giménez-Marqués, R. Mrimi, A. Mielcarek, R. Gref, P. Horcajada, C. Serre and P. Couvreur, Angew. Chem., Int. Ed., 2017, 56, 15565-15569.

168 T. Preiß, A. Zimpel, S. Wuttke and J. Rädler, Materials, 2017, $10,216$.

169 L. Wang, M. Zheng and Z. Xie, J. Mater. Chem. B, 2018, 6, 707-717.

170 S. Wuttke, S. Braig, T. Preiß, A. Zimpel, J. Sicklinger, C. Bellomo, J. O. Rädler, A. M. Vollmar and T. Bein, Chem. Commun., 2015, 51, 15752-15755.

171 Z. Y. Dong, Y. Z. S. Sun, J. Chu, X. Z. Zhang and H. X. Deng, J. Am. Chem. Soc., 2017, 139, 14209-14216.

172 B. Illes, P. Hirschle, S. Baenert, V. Cauda, S. Wuttke and H. Engelke, Chem. Mater., 2017, 29, 8042-8046.

173 B. Illes, S. Wuttke and H. Engelke, Nanomaterials, 2017, 7, 351.

174 R. Li, S. Liu, Q. Tang, T. Wang, Y. Xie and R. Zhai, Z. Anorg. Allg. Chem., 2018, 644, 317-321.

175 H. Zheng, Y. Zhang, L. Liu, W. Wan, P. Guo, A. M. Nyström and X. Zou, J. Am. Chem. Soc., 2016, 138, 962-968.

176 M. Filippousi, S. Turner, K. Leus, P. I. Siafaka, E. D. Tseligka, M. Vandichel, S. G. Nanaki, I. S. Vizirianakis, D. N. Bikiaris, P. V. D. Voort and G. V. Tendeloo, Int. J. Pharm., 2016, 509, 208-218.

177 E. Bellido, T. Hidalgo, M. V. Lozano, M. Guillevic, R. Simón-Vázquez, M. J. Santander-Ortega, 
Á. González-Fernández, C. Serre, M. J. Alonso and P. Horcajada, Adv. Healthcare Mater., 2015, 4, 1246-1257.

178 A. R. Chowdhuri, D. Bhattacharya and S. K. Sahu, Dalton Trans., 2016, 45, 2963-2973.

179 C. Y. Zou, Z. M. Wang, X. M. Ren, R. R. Li, S. P. Zhang and B. Y. Sheng, Inorg. Chem. Commun., 2018, 91, 91-94.

180 Y. A. Li, X. D. Zhao, H. P. Yin, G. J. Chen, S. Yang and Y. B. Dong, Chem. Commun., 2016, 52, 14113-14116.

181 H. Zhang, W. Jiang, R. Liu, J. Zhang, D. Zhang, Z. Li and Y. Luan, ACS Appl. Mater. Interfaces, 2017, 9, 19687-19697. 182 X. Gao, X. Hai, H. Baigude, W. Guan and Z. Liu, Sci. Rep., 2016, 6, 37705.

183 A. S. Abd-El-Aziz, A. A. Abdelghani and A. K. Mishra, J. Inorg. Organomet. Polym. Mater., DOI: 10.1007/s10904-019-01293-y.

184 A.-M. Caminade, A. Ouali, R. Laurent, C.-O. Turrin and J.-P. Majoral, Coord. Chem. Rev., 2016, 308, 478-497.

185 A. Narmani, M. Kamali, B. Amini, A. Salimi and Y. Panahi, Process Biochem., 2018, 69, 178-187.

186 D. T. D. Nguyen, L. G. Bach, T. H. Nguyen, M. H. Ho, M. N. Ho, D. H. Nguyen, C. K. Nguyen and T. T. H. Thi, J. Polym. Res., 2019, 26, 116.

187 M. Maroto-Diaz, N. Sanz del Olmo, L. Muñoz-Moreno, A. M. Bajo, M. J. Carmena, R. Gómez, S. García-Gallego and F. J. de la Mata, Eur. Polym. J., 2019, 113, 229.

188 S. Michlewska, M. Ionov, D. Shcharbin, M. Maroto-Díaz, R. G. Ramirez, F. J. de la Mata and M. Bryszewska, Eur. Polym. J., 2017, 87, 39-47.

189 M. Maroto-Diaz, B. T. Elie, P. Gómez-Sal, J. Pérez-Serrano, R. Gómez, M. Contel and F. J. de la Mata, Dalton Trans., 2016, 45, 7049-7066.

190 M. Gouveia, J. Figueira, M. G. Jardim, R. Castro, H. Tomás, K. Rissanen and J. Rodrigues, Molecules, 2018, 23, 1471.

191 P. Govender, T. Riedel, P. J. Dyson and G. S. Smith, Dalton Trans., 2016, 45, 9529-9539.

192 M. Quadir, S. Fehse, G. Multhaup and R. Haag, Molecules, 2018, 23, 1281.

193 R. Albrecht, S. Fehse, K. Pant, S. Nowag, H. Stephan, R. Haag and C. C. Tzschucke, Macromol. Biosci., 2016, 16, 412-419.

194 S. Rojas, T. Devic and P. Horcajada, J. Mater. Chem. B, 2017, 5, 2560-2573.

195 D. Y. Ma, Z. Li, J. X. Xiao, R. Deng, P. F. Lin, R. Q. Chen, Y. Q. Liang, H. F. Guo, B. Liu and J. Q. Liu, Inorg. Chem., 2015, 54, 6719-6726.

196 H. An, M. Li, J. Gao, Z. Zhang, S. Ma and Y. Chen, Coord. Chem. Rev., 2019, 384, 90-106.

197 S. El Hankari, M. Bousmina and A. El Kadib, Prog. Mater. Sci., 2019, 106, 100579.

198 W. Zhou, L. Wang, F. Li, W. Zhang, W. Huang, F. Huo and H. Xu, Adv. Funct. Mater., 2017, 27, 1605465.

199 F. Cao, E. Ju, C. Liu, W. Li, Y. Zhang, K. Dong, Z. Liu, J. Ren and X. Qu, Nanoscale, 2017, 9, 4128-4134.

200 S. Mignani, J. Rodrigues, H. Tomas, A.-M. Caminade, R. Laurent, X. Shi and J.-P. Majoral, Sci. China Mater., 2018, 61, 1367-1386.
201 L. Chen, S. Mignani, A.-M. Caminade and J.-P. Majoral, Wiley Interdiscip. Rev.: Nanomed. Nanobiotechnol., 2019, 11, e1577.

202 G. I. Dzhardimalieva and I. E. Uflyand, Dalton Trans., 2017, 46, 10139-10176.

203 M. Ghaffari, G. Dehghan, F. Abedi-Gaballu, S. Kashanian, B. Baradaran, J. E. N. Dolatabadi and D. Losic, Eur. J. Pharm. Sci., 2018, 122, 311.

204 N. S. Sommerfeld, M. Hejl, M. H. M. Klose, E. SchreiberBrynzak, A. Bileck, S. M. Meier, C. Gerner, M. A. Jakupec, M. Galanski and B. K. Keppler, Eur. J. Inorg. Chem., 2017, 2017, 1713-1720.

205 K. Dave and V. V. K. Venuganti, Ther. Delivery, 2017, 8, 1077-1096.

206 H. L. Che, M. Huo, L. Peng, Q. Q. Ye, J. Guo, K. Wang, Y. Wei and J. Y. Yuan, Polym. Chem., 2015, 6, 2319-2326.

207 W. Cai, J. Wang, C. Chu, W. Chen, C. Wu and G. Liu, Adv. Sci., 2019, 6, 1801526.

208 K. Fujita, Y. Tanaka, S. Abe and T. Ueno, Angew. Chem., Int. Ed., 2016, 55, 1056-1060.

209 D. Nguyen, T. Nguyen, S. A. Rice and C. Boyer, Biomacromolecules, 2015, 16, 2776-2786.

210 X. Li, J. Mu, F. Liu, E. W. P. Tan, B. Khezri, R. D. Webster, E. K. L. Yeow and B. G. Xing, Bioconjugate Chem., 2015, 26, 955-961.

211 F. Li, T. Li, W. Cao, L. Wang and H. Xu, Biomaterials, 2017, 133, 208-218.

212 S. Ding and U. Bierbach, Future Med. Chem., 2015, 7, 911927.

213 H. Song, X. Kang, J. Sun, X. Jing, Z. Wang, L. Yan, R. Qi and M. Zheng, Chem. Commun., 2016, 52, 2281-2283.

214 H. Song, W. Li, R. Qi, L. Yan, X. Jing, M. Zheng and H. Xiao, Chem. Commun., 2015, 51, 11493-11495.

215 D. Zhou, J. Guo, G. B. Kim, J. Li, X. Chen, J. Yang and Y. Huang, Adv. Healthcare Mater., 2016, 5, 2493-2499.

216 Y. R. Zheng, K. Suntharalingam, T. C. Johnstone and S. J. Lippard, Chem. Sci., 2015, 6, 1189-1193.

217 M. Ma, T. Luan, M. Yang, B. Liu, Y. Wang, W. An, B. Wang, R. Tang and A. Hao, Soft Matter, 2017, 13, 1534-1538.

218 A. Khan, L. Wang, H. Yu, M. Haroon, R. S. Ullah, A. Nazir, T. Elshaarani, M. Usman, S. Fahad and F. Haq, Appl. Organomet. Chem., 2018, 32, e4575.

219 E. Wajs, T. T. Nielsen, K. L. Larsen and A. Fragoso, Nano Res., 2016, 9, 2070-2078.

220 L. Peng, H. Zhang, A. Feng, M. Huo, Z. Wang, J. Hu, W. Gao and J. Yuan, Polym. Chem., 2015, 6, 3652-3659.

221 S. Szunerits, F. Teodorescu and R. Boukherroub, Eur. Polym. J., 2016, 83, 467-477.

222 S. Mu, Q. Ling, X. Liu, J. Ruiz, D. Astruc and H. Gu, J. Inorg. Biochem., 2019, 193, 31-41.

223 D. Scheid, M. von der Lühe and M. Gallei, Macromol. Rapid Commun., 2016, 37, 1573.

224 L. Liu, L. Rui, Y. Gao and W. Zhang, Polym. Chem., 2015, 6, 1817-1829.

225 F. Xu, H. Li, Y.-L. Luo and W. Tang, ACS Appl. Mater. Interfaces, 2017, 9, 5181-5192. 
226 J. Morsbach, J. Elbert, C. Rüttiger, S. Winzen, H. Frey and M. Gallei, Macromolecules, 2016, 49, 3406-3414.

227 P. Shi, Y. Qu, C. Liu, H. Khan, P. Sun and W. Zhang, ACS Macro Lett., 2016, 5, 88-93.

228 T. Noyhouzer, C. L'Homme, I. Beaulieu, S. Mazurkiewicz, S. Kuss, H.-B. Kraatz, S. Canesi and J. Mauzeroll, Langmuir, 2016, 32, 4169-4178.

229 S. J. T. Rezaei, V. Amani, M. R. Nabid, N. Safari and H. Niknejad, Polym. Chem., 2015, 6, 2844-2853.

230 Y. Cong, H. Xiao, H. Xiong, Z. Wang, J. Ding, C. Li, X. Chen, X.-J. Liang, D. Zhou and Y. Huang, Adv. Mater., 2018, 30, 1706220.

231 H. J. Li, J. Z. Du, J. Liu, X. J. Du, S. Shen, Y. H. Zhu, X. Y. Wang, X. D. Ye, S. M. Nie and J. Wang, ACS Nano, 2016, 10, 6753-6761.

232 S. Shen, H. J. Li, K. G. Chen, Y. C. Wang, X. Z. Yang, Z. X. Lian, J. Z. Du and J. Wang, Nano Lett., 2017, 17, 3822-3829.

233 G.-M. Bao, L. Wang, H.-Q. Yuan, X.-Y. Wang, T.-X. Mei and M.-R. Qu, RSC Adv., 2016, 6, 109453-109459.

234 X. Chen, Z. Shi, R. Tong, S. Ding, X. Wang, J. Wu, Q. Lei and W. Fang, ACS Biomater. Sci. Eng., 2018, 4, 4183-4192.

235 L. Tang, J. F. Shi, X. L. Wang, S. H. Zhang, H. Wu, H. F. Sun and Z. Y. Jiang, Nanotechnology, 2017, 28, 275601.

236 M. Ni, N. Zhang, W. Xia, X. Wu, C. Yao, X. Liu, X.-Y. Hu, C. Lin and L. Wang, J. Am. Chem. Soc., 2016, 138, 66436649.

237 J. H. Kim, H. Eguchi, M. Umemura, I. Sato, S. Yamada, Y. Hoshino, T. Masuda, I. Aoki, K. Sakurai, M. Yamamoto and Y. Ishikawa, NPG Asia Mater., 2017, 9, e367.

238 Y. Y. Huang, W. Huang, L. Chan, B. W. Zhou and T. F. Chen, Biomaterials, 2016, 103, 183-196.

239 W.-H. Chen, S. Y. Sung, M. Fadeev, A. Cecconello, R. Nechushtai and I. Willner, Nanoscale, 2018, 10, 46504657.

240 X. Zhu and C.-Q. Wang, J. Colloid Interface Sci., 2016, 480, 39-48.

241 Y. Kang, X. Ju, L. S. Ding, S. Zhang and B. J. Li, ACS Appl. Mater. Interfaces, 2017, 9, 4475-4484.

242 C. Zuo, X. Y. Dai, S. J. Zhao, X. N. Liu, S. L. Ding, L. W. Ma, M. Z. Liu and H. Wei, ACS Macro Lett., 2016, 5, 873-878.

243 X.-M. Zhang, K. Guo, L.-H. Li, S. Zhang and B.-J. Li, J. Mater. Chem. B, 2015, 3, 6026-6031.

244 G. Y. Li, Z. R. Dong, Y. T. Zhu, W. J. Tong and C. Y. Gao, J. Colloid Interface Sci., 2016, 475, 196-202.

245 Y. Kang, Y. Ma, S. Zhang, L. S. Ding and B. J. Li, ACS Macro Lett., 2015, 4, 543-547.

246 S. Wang, C. Yao, M. Ni, Z. Xu, M. Cheng, X.-Y. Hu, Y.-Z. Shen, C. Lin, L. Wang and D. Jia, Polym. Chem., 2017, 8, 682-688.

247 S. Behzadi, M. Gallei, J. Elbert, M. Appold, G. Glasser, K. Landfester and D. Crespy, Polym. Chem., 2016, 7, 34343443.

248 Y. Chang, C. Hou, J. Ren, X. Xin, Y. Pei, Y. Lu, S. Cao and Z. Pei, Chem. Commun., 2016, 52, 9578-9581.

249 O. Mergel, S. Schneider, R. Tiwari, P. T. Kühn, D. Keskin, M. C. A. Stuart, S. Schöttner, M. de Kanter, M. Noyong,
T. Caumanns, J. Mayer, C. Janzen, U. Simon, M. Gallei, D. Wöll, P. van Rijn and F. A. Plamper, Chem. Sci., 2019, 10, 1844-1856.

250 B. V. K. J. Schmidt, D. Kugele, J. von Irmer, J. Steinkoenig, H. Mutlu, C. Rüttiger, C. J. Hawker, M. Gallei and C. BarnerKowollik, Macromolecules, 2017, 50, 2375-2386.

251 S. Nagata, K. Kokado and K. Sada, Chem. Commun., 2015, 51, 8614-8617.

252 M. H. Teplensky, M. Fantham, P. Li, T. C. Wang, J. P. Mehta, L. J. Young, P. Z. Moghadam, J. T. Hupp, O. K. Farha, C. F. Kaminski and D. Fairen-Jimenez, J. Am. Chem. Soc., 2017, 139, 7522-7532.

253 W. X. Lin, Q. Hu, J. C. Yu, K. Jiang, Y. Y. Yang, S. C. Xiang, Y. J. Cui, Y. Yang, Z. Y. Wang and G. D. Qian, ChemPlusChem, 2016, 81, 804-810.

254 K. Jiang, L. Zhang, Q. Hu, D. Zhao, T. F. Xia, W. X. Lin, Y. Y. Yang, Y. J. Cui, Y. Yang and G. D. Qian, J. Mater. Chem. B, 2016, 4, 6398-6401.

255 E. Lashkari, H. Wang, L. S. Liu, J. Li and K. Yam, Food Chem., 2017, 221, 926-935.

256 L. L. Tan, H. Li, Y. Zhou, Y. Zhang, X. Feng, B. Wang and Y. W. Yang, Small, 2015, 11, 3807-3813.

257 X. S. Meng, B. Gui, D. Q. Yuan, M. Zeller and C. Wang, Sci. Adv., 2016, 2, e1600480.

258 C. Adhikari and A. Chakraborty, ChemPhysChem, 2016, 7, 1070-1077.

259 X. G. Wang, Z. Y. Dong, H. Cheng, S. S. Wan, W. H. Chen, M. Z. Zou, J. W. Huo, H. X. Deng and X. Z. Zhang, Nanoscale, 2015, 7, 16061-16070.

260 S. Y. Tan, C. Y. Ang, A. Mahmood, Q. Y. Qu, P. Z. Li, R. Q. Zou and Y. L. Zhao, ChemNanoMat, 2016, 2, 504-508.

261 H.-J. Cai, T.-T. Shen, J. Zhang, C.-F. Shan, J.-G. Jia, X. Li, W.-S. Liu and Y. Tang, J. Mater. Chem. B, 2017, 5, 23902394.

262 W.-H. Chen, W.-C. Liao, Y. S. Sohn, M. Fadeev, A. Cecconello, R. Nechushtai and I. Willner, Adv. Funct. Mater., 2018, 28, 1705137.

263 S. Wadhwa and R. J. Mumper, Crit. Rev. Ther. Drug Carrier Syst., 2015, 32, 215-245.

264 M. Li, Z. Tang, S. Lv, W. Song, H. Hong, X. Jing, Y. Zhang and X. Chen, Biomaterials, 2014, 35, 3851-3864.

265 A. Gandioso, E. Shaili, A. Massaguer, G. Artigas, A. González-Cantó, J. A. Woods, P. J. Sadler and V. Marchán, Chem. Commun., 2015, 51, 9169-9172.

266 S. Li, C. Li, S. Jin, J. Liu, X. Xue, A. S. Eltahan, J. Sun, J. Tan, J. Dong and X. J. Liang, Biomaterials, 2017, 144, 119-129.

267 C. Poon, C. He, D. Liu, K. Lu and W. Lin, J. Controlled Release, 2015, 201, 90-99.

268 M. Callari, S. Wong, H. Lu, J. Aldrich-Wright, P. de Souza and M. H. Stenzel, Polym. Chem., 2017, 8, 6289-6299.

269 W. Song, Z. Tang, D. Zhang, Y. Zhang, H. Yu, M. Li, S. Lv, H. Sun, M. Deng and X. Chen, Biomaterials, 2014, 35, 3005-3014.

270 D. Zhou, S. He, Y. Cong, Z. Xie, X. Chen, X. Jing and Y. Huang, J. Mater. Chem. B, 2015, 3, 4913-4921.

271 S. He, Y. Cong, D. Zhou, J. Li, Z. Xie, X. Chen, X. Jing and Y. Huang, J. Mater. Chem. B, 2015, 3, 8203-8211. 
272 D. Zhou, Y. Cong, Y. Qi, S. He, H. Xiong, Y. Wu, Z. Xie, X. Chen, X. Jing and Y. Huang, Biomater. Sci., 2015, 3, 182-191.

273 R. Qi, H. Xiao, S. Wu, Y. Li, Y. Zhang and X. Jing, J. Mater. Chem. B, 2015, 3, 176-179.

274 T. Wang, G. Sun, M. Wang, B. Zhou and J. Fu, ACS Appl. Mater. Interfaces, 2015, 7, 21295-21304.

275 E. Baa, G. M. Watkins, R. W. Krause and D. N. Tantoh, Chin. J. Chem., 2019, 37, 378-404.

276 S. Rojas, A. Arenas-Vivo and P. Horcajada, Coord. Chem. Rev., 2019, 388, 202-226.

277 Y. Ma, Q. Mou, D. Wang, X. Zhu and D. Yan, Theranostics, 2016, 6, 930-947.

278 S. Chen, Q. Lei, W.-X. Qiu, L.-H. Liu, D.-W. Zheng, J.-X. Fan, L. Rong, Y.-X. Sun and X.-Z. Zhang, Biomaterials, 2017, 117, 92-104.

279 Y. K. Li, Y. C. Li, X. Zhang, X. H. Xu, Z. J. Zhang, C. Hu, Y. Y. He and Z. W. Gu, Theranostics, 2016, 6, 1293-1305.

280 G. Yu, M. Zhang, M. L. Saha, Z. Mao, J. Chen, Y. Yao, Z. Zhou, Y. Liu, C. Gao, F. Huang, X. Chen and P. J. Stang, J. Am. Chem. Soc., 2017, 139, 15940-15949.

281 H.-X. Zhao, Q. Zou, S.-K. Sun, C. Yu, X. Zhang, R.-J. Li and Y.-Y. Fu, Chem. Sci., 2016, 7, 5294-5301.

282 R. Bian, T. Wang, L. Zhang, L. Li and C. Wang, Biomater. Sci., 2015, 3, 1270-1278.

283 C. J. Zhang, Q. L. Hu, G. X. Feng, R. Y. Zhang, Y. Y. Yuan, X. M. Lu and B. Liu, Chem. Sci., 2015, 6, 4580-4586.
284 W. Liu, Y. Wang, Y. Li, S. Cai, X. Yin, X. He and Y. Zhang, Small, 2017, 13, 1603459.

285 J. Liu, L. Zhang, J. Lei, H. Shen and H. Ju, ACS Appl. Mater. Interfaces, 2017, 9, 2150-2158.

286 S. Y. Li, H. Cheng, W. X. Qiu, L. Zhang, S. S. Wan, J. Y. Zeng and X. Z. Zhang, Biomaterials, 2017, 142, 149-161.

287 D. D. Wang, J. J. Zhou, R. H. Chen, R. H. Shi, G. Z. Zhao, G. L. Xia, R. Li, Z. B. Liu, J. Tian, H. J. Wang, Z. Guo, H. B. Wang and Q. W. Chen, Biomaterials, 2016, 100, 27-40.

288 J. C. Yang, Y. Chen, Y. H. Li and X. B. Yin, ACS Appl. Mater. Interfaces, 2017, 9, 22278-22288.

289 Y. D. Zhu, S. P. Chen, H. Zhao, Y. Yang, X. Q. Chen, J. Sun, H. S. Fan and X. D. Zhang, ACS Appl. Mater. Interfaces, 2016, 8, 34209-34217.

290 X. Chen, M. Zhang, S. Li, L. Li, L. Zhang, T. Wang, M. Yu, Z. Mou and C. Wang, J. Mater. Chem. B, 2017, 5, 1772-1778.

291 J. Y. Zeng, M. K. Zhang, M. Y. Peng, D. Gong and X. Z. Zhang, Adv. Funct. Mater., 2018, 28, 1705451.

292 K. Lu, C. He, N. Guo, C. Chan, K. Ni, R. R. Weichselbaum and W. Lin, J. Am. Chem. Soc., 2016, 138, 12502-12510.

293 J. Y. Zeng, M. Z. Zou, M. Zhang, X. S. Wang, X. Zeng, H. Cong and X. Z. Zhang, ACS Nano, 2018, 12, 4630-4640.

294 S.-Y. Li, H. Cheng, B.-R. Xie, W.-X. Qiu, J.-Y. Zeng, C.-X. Li, S.-S. Wan, L. Zhang, W.-L. Liu and X.-Z. Zhang, ACS Nano, 2017, 11, 7006-7018.

295 S. Aghevlian, Y. Lu, M. A. Winnik, D. W. Hedley and R. M. Reilly, Mol. Pharmaceutics, 2018, 15, 1150-1159. 